\title{
Shared knowledge in complex teams
}

\author{
Citation for published version (APA):
}

Sander, P. C. (2016). Shared knowledge in complex teams: an investigation of the shared mental model construct. [Doctoral Thesis, Maastricht University]. Maastricht University. https://doi.org/10.26481/dis.20160331ps

Document status and date:

Published: 01/01/2016

DOI:

10.26481/dis.20160331ps

Document Version:

Publisher's PDF, also known as Version of record

\section{Please check the document version of this publication:}

- A submitted manuscript is the version of the article upon submission and before peer-review. There can be important differences between the submitted version and the official published version of record.

People interested in the research are advised to contact the author for the final version of the publication, or visit the DOI to the publisher's website.

- The final author version and the galley proof are versions of the publication after peer review.

- The final published version features the final layout of the paper including the volume, issue and page numbers.

Link to publication

\footnotetext{
General rights rights.

- You may freely distribute the URL identifying the publication in the public portal. please follow below link for the End User Agreement:

www.umlib.nl/taverne-license

Take down policy

If you believe that this document breaches copyright please contact us at:

repository@maastrichtuniversity.nl

providing details and we will investigate your claim.
}

Copyright and moral rights for the publications made accessible in the public portal are retained by the authors and/or other copyright owners and it is a condition of accessing publications that users recognise and abide by the legal requirements associated with these

- Users may download and print one copy of any publication from the public portal for the purpose of private study or research.

- You may not further distribute the material or use it for any profit-making activity or commercial gain

If the publication is distributed under the terms of Article $25 \mathrm{fa}$ of the Dutch Copyright Act, indicated by the "Taverne" license above, 


\section{Shared Knowledge in Complex Teams}

An Investigation of the Shared Mental Model Construct 
The research reported here was a cooperation between

and

\section{Maastricht University in Learning!}

and part of the research project Human Factors in Future Air Traffic Management of the

\section{knowledge \& development centre}

Colophon

Cover design: Arash Kathami

Production: Wilhelm Brüggemann Buchbinderei und Druckerei GmbH

Copyright @ 2016 P. Sander, Bremen 


\section{Shared Knowledge in Complex Teams}

\section{An Investigation of the Shared Mental Model Construct}

\section{DISSERTATION}

To obtain the degree of Doctor at Maastricht University, on the authority of Rector Magnificus, Prof dr. L. L. G. Soete, in accordance with the decision of the Boards of Deans, to be defended in public on

Thursday 31 March 2016 at 12.00 p.m.

by

Pia Christina Sander (née Justen) 


\section{Supervisor:}

Prof. dr. F. R. H. Zijlstra

\section{Co-supervisor:}

Dr. R. R. A. van Doorn

Dr. J. van der Pal

\section{Assessment committee:}

Prof. dr. R. A C. Ruiter (chair)

Prof. dr. Gudela Grote (ETH Zürich)

Prof. dr. em. R. Roe

Prof. dr. Piet Van den Bossche (University of Antwerp)

Prof. dr. J. J. G. Van Merriënboer 


\section{Table of Contents}

Chapter 1

Introduction

Chapter 2

Identifying shared mental models within teams of air traffic controllers: A cognitive task analysis

Chapter 3

A card sorting study to investigate shared mental models in air traffic control teams as a function of organizational context and work experience

Chapter 4

Knowledge sharing facilitates a team's shared mental models, communication and performance

Chapter 5

When team members communicate via datalink: The positive effects of guided off-task knowledge sharing on shared mental models, team behaviour and team performance

Chapter 6

Team adaptation to an unforeseen system failure: Limits of the potential aids of shared knowledge and standardized communication

Chapter 7

General discussion and conclusion

Summary.

Samenvatting

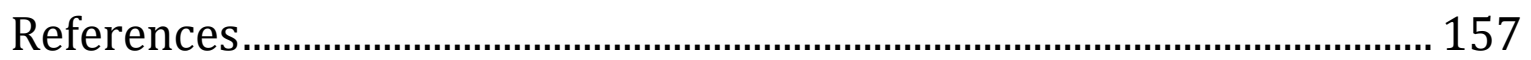

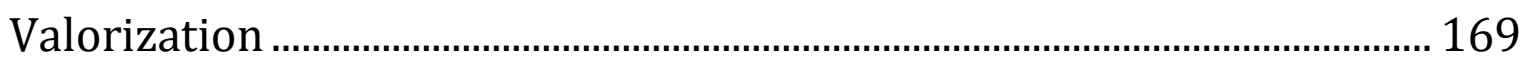

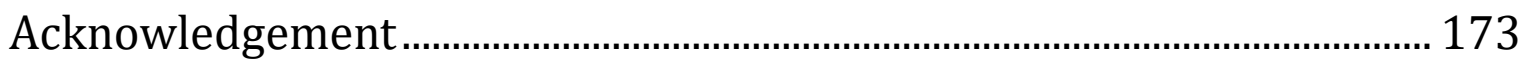

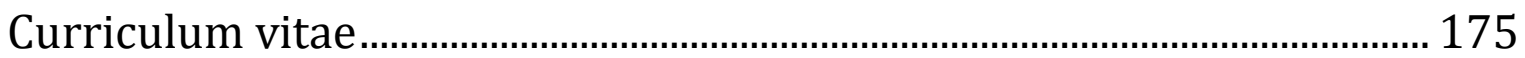



Chapter 1

\section{Introduction}



It is a winter's afternoon at a European airport. The air traffic control (ATC) tower is staffed with a team of nine air traffic controllers (ATCOs) and assistants who are jointly responsible for a safe and efficient traffic flow. It is relatively quiet so that not every working positions is manned and some controllers are on hold. Suddenly the weather changes. The temperature drops, visibility decreases and a large snow cloud approaches the area around the airport. Such weather conditions usually lead to high workload and the tower supervisor decides that the entire team is required on duty, so everyone takes her/his position.

To respond to the developing situation the tower supervisor together with area control, airport operation management, and the metrological advisor, develop a plan for the next two hours in which heavy snowfall is expected. The tower supervisor goes back to brief his team, explains the plan, distributes responsibilities and ensures that all team members are on the same page. The team needs to adapt quickly to the new situation and the plan. Each team member works hard to keep the traffic flow smooth and efficient, being responsible for different and interdependent parts in the dynamic chain: start-up clearance, de-icing, taxing, take-off and landing, and the coordination of ground vehicles. These individual tasks are already demanding so that coordination among team members must be smooth and effortless to keep the total workload tolerable. Some controllers call this way of teamwork 'elbow coordination'.

The supervisor receives a call that an adjacent airport has closed due to the snow, which has two major consequences: controllers cannot clear outbound traffic to that particular airport and must expect additional inbound traffic including an A380 aircraft. This information is crucial for all positions in the tower and the supervisor walks by every staff member to communicate the information and give instructions specific to each position. The approaching A380 causes some stress since controllers are not familiar with the procedures for the unusual landing of an aircraft of this size. The supervisor assistant proactively consults the operational manual and provides the relevant information to the involved team members.

Two of the team members on hold recognise that the start-up and delivery controller have worked intensively for more than 45 minutes and offer to take over their positions. While switching positions, three pilots call for start-up clearance and request for de-icing of the aircraft. The new controllers are immediately absorbed in the situation and the two former controllers, happy to have a break, leave without informing the others of the closure of the adjacent airport. A little later the ground controller suddenly realises that several aircraft have been cleared to the closed airport. She calls in the group so that everyone is aware of the situation. The responsible controller corrects the mistake by giving alternative clearances, while trying to keep the inefficiency and delays to a minimum. The joint effort of all team members eventually results in all aircraft leaving and reaching the airport on time. Slowly the weather calms down and team members start to relax after two intensive hours of work. The supervisor praises the team for great teamwork and good performance. 
This fictive situation is just a glance at the work of ATCOs and illustrates the high complexity of their tasks and the many interdependencies among team members. Although ATCOs have distinctive responsibilities, they function as a team, and it is important that they have a shared understanding of each other's behaviour and of the situation. In the example described, a lack of a shared understanding led to the situation that controllers cleared aircraft to a closed airport. Once team members had shared the information in the team, they quickly adapted to the situation and jointly worked towards a common solution.

ATC and other highly dynamic and safety critical work environments require team members who effectively exchange relevant information, share knowledge, and understand each other intuitively (Salas \& Fiore, 2004). Shared knowledge between ATCOs help them to jointly work towards a common goal under high workload and excessive time pressure (Cooke, Kieckel, \& Helm, 2001). This shared knowledge is also referred to as a shared mental model (SMM), which is the common knowledge between members of a team that help them to coordinate their actions and work together effectively (Cannon-Bowers, Salas, \& Converse, 1993). Due to the high importance of similar and accurate knowledge structures in complex team environments, this dissertation aims to increase the understanding of SMMs in complex teams and specifically teams of ATCOs. The studies focus on what types of mental models are relevant in ATC and how SMMs may contribute to safe and efficient air transportation.

To better understand SMMs the studies in this dissertation address a number of unresolved issues in the SMM literature. First, the literature lacks a thorough understanding of SMMs in specific and complex teams. Such an understanding is important as complex team and task contexts require a number of separate and specific types of SMMs (Badke-Schaub, Neumann, Lauche, \& Mohammed, 2007; Mohammed, Ferzandi, \& Hamilton, 2010). ATCO teams are particularly interesting for this research as these teams can only function on the basis of interdependent tasks and responsibilities. A better understanding of the shared knowledge in these teams may help to improve ATC and will contribute to the SMM literature. This dissertation therefore aims to adjust and expand existing typologies of SMMs (Cannon-Bowers et al., 1993; Mathieu, Heffner, Goodwin, Cannon Bowers, \& Salas, 2005) according to the complex knowledge requirements in ATC.

A second unresolved issue in the SMM literature concerns how individual mental models develop into SMMs (Kennedy \& McComb, 2010). It is especially unclear how organizations can support team members in the development of effective SMMs (Langan-Fox, Anglim, \& Wilson, 2004). Such support is critical as effective SMMs do not necessarily develop due to mere task experience (McComb, 2007). Instead, team members need to exchange information and share knowledge actively with each other (Uitdewilligen, Waller, \& Zijlstra, 2010). In dynamic teams, such as in teams of ATCOs, possibilities are often limited for such information and knowledge exchange (Malakis, Kontogiannis, \& Kirwan, 2010b; Waller, Gupta, \& Giambatista, 2004). The dynamic task usually requires full attention and leaves little opportunities to communicate information that is not 
directly relevant for immediate task execution. This dissertation therefore investigates whether team members develop more effective shared knowledge when they are guided in mutually exchanging information (De Vries \& Van den Hooff, 2006).

The third unresolved issue in SMM research that this dissertation addresses is the role of SMMs in team adaptation. The ability to adapt is critical in ATC and other dynamic environments because situations change quickly and often unexpectedly (Malakis et al., 2010b). The example scenario above describes how ATC teams need to adapt to changing weather and the resulting altered status of nearby airports. Effective adaptation to such changes involves that team members quickly apply alternate cooperation and task strategies. Their SMMs may help team members in adapting to such changes (Burke, Stagl, Salas, Pierce, \& Kendall, 2006; Uitdewilligen, Waller, \& Pitariu, 2013). To study whether SMM positively affect team adaptation, this dissertation investigates team members' adaptive performance before and after an unforeseen change(Lang \& Bliese, 2009)(Lang \& Bliese, 2009)(Lang \& Bliese, 2009)(Lang \& Bliese, 2009)(Lang \& Bliese, 2009)(Lang \& Bliese, 2009)(Lang \& Bliese, 2009)(Lang \& Bliese, 2009)(Lang \& Bliese, 2009)(Lang \& Bliese, 2009).

Team behaviour and particularly communication play a critical role in dynamic and complex teams (Waller et al., 2004; Zijlstra, Waller, \& Phillips, 2012) and therefore formed an important aspect in studies of this dissertation. Communication is both a potential facilitator and indicator of the positive contribution of SMMs to team performance. Communication facilitates SMMs as team members who communicate essential information also align their knowledge and cultivate both the emergence and maintenance of SMMs (Burke et al., 2006; McComb, 2007; Zijlstra et al., 2012). Communication within a team can also be an indicator of SMMs as it is possible to measure SMMs by analysing communication as part of a team's behaviour (Kennedy \& McComb, 2010; Salas, Rosen, Burke, Nicholson, \& Howse, 2007). Team members who have developed SMMs usually communicate more concisely and provide information proactively and before other team members request this information (Salas, Rosen, et al., 2007).

The following parts of this introduction will explain the theoretical background of the dissertation. This starts with a description of the SMM concept, including a definition, the differentiation to other cognitive team concepts and a short summary of the measurement methods. The introduction proceeds with an overview of the different types and properties of SMMs. Afterwards, it introduces notions on SMM development and propose knowledge sharing as possible support for the development of SMMs. Lastly, the introduction describes the relationship of SMMs with team effectiveness and specifically team adaption, and the relationship of SMMs with communication. In the descriptions, I regularly refer to the scenario of ATC described above to illustrate the practical relevance of this research. The theoretical background closes with the presentation of a conceptual model that clarifies how SMMs are approached in the different studies of this research. The introduction ends with an overview of the research questions and the structure of the remaining dissertation. 


\section{The Shared Mental Model Concept}

ATCOs in the example outlined above performed successfully because they effectively and purposefully combined their efforts. They enriched their joint knowledge base by combining individual expertise, and simultaneously maintained the distinct knowledge required for the specific roles and responsibilities they have in the team. The scientific and practical need to explain these properties generally found in effective teams, led to the emergence of the construct of SMMs (Cannon-Bowers et al., 1993). SMMs stand for the cognitive structures and mechanisms that make smooth coordination possible and that enable team members to understand both task and team members' demands without explicit communication (Cannon-Bowers, Salas, Blickensderfer, \& Bowers, 1998).

The description of the SMM construct was strongly influenced by research on individual mental models. These individual mental models are defined as knowledge structures that allow individuals to describe situations, understand and explain events, and predict and anticipate actions or behaviour in a given system (Johnson-Laird, 1983; Rouse \& Morris, 1986). With increasing task complexity these individual mental models must be shared between several individuals who usually have to work together in a team. Those team members must align their mental models in order to coordinate their activities effectively (Cannon-Bowers et al., 1993). The cognitive match of team members' mental models is referred to as SMMs and thus assumes that this collective cognitive property arises from individual knowledge of the team members (Kozlowski \& Ilgen, 2000).

SMMs have been defined as:

"knowledge structures held by members of a team that enable them to form accurate explanations and expectations for the task, and, in turn to coordinate their actions and adapt their behavior to demands of the task and other team members." (Cannon-Bowers et al., 1993, p. 228)

That is, team members have a shared understanding about present and future situations, and interpret and describe these situations similarly. Team members who share knowledge about the task and the team are also assumed to be able to integrate their efforts, coordinate their actions, anticipate each other's needs and adapt their behaviours accordingly (Klimoski \& Mohammed, 1994; Levesque, Wilson, \& Wholey, 2001). In turn, these synchronized team behaviours facilitate decision making and team functioning in complex and dynamic environments (Cannon-Bowers, Salas, et al., 1998; Cannon-Bowers et al., 1993). These notions are the basis for SMM Theory that assumes a positive relationship between shared knowledge, team behaviours, and team performance (Klimoski \& Mohammed, 1994; Kraiger \& Wenzel, 1997).

There are three both practical and theoretical reasons to study SMMs in diverse task environments and teams (Cannon-Bowers \& Salas, 2001). First, SMMs have predictive power in anticipating team member and team effectiveness. Predictions about a team's effectiveness are possible by examining team members' SMMs. This can be useful in work domains that require frequent composition of new teams (e.g. project teams) or in situations that require team members to 
readily work together despite little teamwork experience (e.g. crisis management teams). Second, SMMs have explanatory power, as the construct contributes to the understanding of effective teamwork mechanisms. Team members who hold SMMs differ in several ways from team members with less effective shared knowledge. For example, team members with effective SMMs can coordinate their actions implicitly without the need for overt communication (Cannon-Bowers \& Salas, 2001); they engage in active information sharing during non-routine conditions to develop a common picture of the situation (Waller et al., 2004); and they show more back-up behaviour in terms of monitoring each other's performance and offering assistance if needed (Salas, Rosen, et al., 2007; Smith-Jentsch, 2009). These latter behaviours that are associated with SMMs can help to explain differences between successful and unsuccessful team performance. The third reason to study SMMs in diverse task environment and teams has to do with improving performance of less well functioning teams. Indeed, SMMs have caught interest in research for their diagnostic power to identify coordination and performance difficulties that teams may experience. For example, poor coordination, ineffective information exchange, or a lack of mutual understanding can indicate that team members are 'not on the same page'. Being able to detect such problems and understand their causes is essential in resolving performance issues and helping team members to develop a common knowledge base. This means that identifying the underlying mental models of team behaviour can be an important initial step towards changing ineffective behaviours (Druskat \& Pescosolido, 2002).

Motivated by the practical and theoretical relevance of the SMM concept, a substantial number of studies, reviews and book chapters have been published on SMMs in the last 20 years (Banks \& Millward, 2000; Bolstand \& Endsley, 1999; Cannon-Bowers et al., 1993; Cooke, Gorman, Duran, \& Taylor, 2007; DeChurch \& Mesmer-Magnus, 2010b; Klimoski \& Mohammed, 1994; Kraiger \& Wenzel, 1997; Langan-Fox, Wirth, Code, Langfield-Smith, \& Wirth, 2001; Mathieu, Heffner, Goodwin, Salas, \& Cannon Bowers, 2000; Mohammed et al., 2010; Resick, Dickson, Mitchelson, Allison, \& Clark, 2010; Salas \& Fiore, 2004; Smith-Jentsch, Campbell, Milanovich, \& Reynolds, 2001; Stout, Cannon Bowers, Salas, \& Milanovich, 1999; Uitdewilligen et al., 2010; Van den Bossche, Gijselaers, Segers, Woltjer, \& Kirschner, 2011; Waller et al., 2004). Researchers have reached considerable agreement about the definition of SMMs and the conceptual differentiation in regard to other forms of shared cognition, such as transactive memory ${ }^{1}$ or shared beliefs ${ }^{2}$. The general difference is that most concepts of team cognition focus on either knowledge related to the task (e.g. transactive memory), or on teamwork (e.g. group learning; Edmondson, 1997), whereas SMM has a broader scope, which includes both task and team knowledge (Mohammed et al., 2010).

Less consensus exists on the measurement of SMMs (DeChurch \& Mesmer-Magnus, 2010b). Several methodologies have been applied to measure SMMs, varying from pairwise comparison

\footnotetext{
${ }^{1}$ Transactive memory is "the knowledge held by individual group members coupled with an awareness of the distribution of that knowledge" (Smith-Jentsch, Kraiger, Cannon-Bowers, \& Salas, 2009, p. 182).

${ }^{2}$ Shared beliefs are tacit beliefs common among individuals about, for example, how to respond to mistakes (Cannon \& Edmondson, 2001).
} 
Chapter 1

ratings (Cooke, Gorman, Duran, et al., 2007), scenario ratings (Mathieu, Rapp, Maynard, \& Mangos, 2010), card sorting methods (Smith-Jentsch, Cannon-Bowers, Tannenbaum, \& Salas, 2008), multidimensional scaling (Rentsch \& Klimoski, 2001) and questionnaires (Espevik, Johnsen, Eid, \& Thayer, 2006). But a comparison of concept-mapping, pairwise comparison ratings and causal mapping has put the convergent validity of these measurement methods into question (Ross \& Allen, 2012). That is why the measurement of SMMs is the "most significant challenge to the progress of this area" (Mohammed et al., 2010, p. 881) and every method has strengths and weaknesses and may reveal different findings. Especially the strength of the relationship between SMMs and team processes depends on how SMMs are operationalized (DeChurch \& Mesmer-Magnus, 2010b). The choice of an adequate measurement method should therefore be based on the task and type of teams studied, as well as on the focus of the research. Accordingly, in this dissertation, three different measures for SMMs are used that were chosen carefully based on the specific aims of the individual studies.

\section{Multiple Types of Shared Mental Models}

By building on theory and research of individual knowledge structures, it is often assumed that team members hold multiple types of mental models that enable them to understand and explain complex tasks (Rouse \& Morris, 1986). In the ATC scenario described earlier, team members must actively share new knowledge on the landing procedure of an A380 aircraft. They relieve and offer support to colleagues as they have a similar understanding about each other's workload; and they enable effective traffic flow because they have a shared situational awareness.

Various SMM types have been investigated based on a taxonomy originally proposed by Cannon-Bowers and colleagues (1993). They suggested a theoretical framework that distinguishes four mental models types that team members generally share, namely the equipment model, the task model, the team model, and the team interaction model. Such a framework maintains that, first, team members should have an accurate understanding of the equipment and systems they work with. In complex team environments, human-machine-interaction substantially contributes to most task execution and thus requires common knowledge about the role, use, and limitation of the equipment. Moreover, a shared awareness of system states is especially important for highly automated systems because information is not always transparent, and thus often prone to different interpretation. The second assertion of the framework is that team members must share knowledge about the job and task. Team members should have a common understanding about task procedures, rules, and strategies and interpret environmental constraints regarding task execution in a similar way. The third model of the framework, the team interaction model, includes knowledge about how roles and responsibilities are distributed among team members, how information is exchanged and what communication channels are used. This model comprises shared knowledge about typical interaction patterns and the information flow between team members. The framework finally proposes the so-called team mental 
model that represents mutual awareness about the skills, attitudes and knowledge of other team members. This model specifies the shared knowledge about personal preferences and tendencies of the individual members. Cannon-Bowers and colleagues (1993) suggested that, although the framework's four model types represent separate content domains, these models are also interrelated. For example, communication patterns are formalized in procedures in ATC and thus must combine knowledge from the task and the team interaction models.

Although the subdivision in four mental model types has been cited by many researchers and is conceptually well described, empirical evidence is still lacking. From a practical point of view, it is challenging to examine multiple mental models in a single study because interdependencies among knowledge elements exist (Mathieu et al., 2000). This may be the reason why most researchers choose to combine the four model types into two major content domains, namely task-related and teamrelated models (e.g. M. Lee \& Johnson, 2008; Lim \& Klein, 2006; Mathieu et al., 2005). The former integrates knowledge about the equipment and the task, whereas the latter focuses on knowledge about team members and team interaction. While the choice to compose multiple mental model types into two content domains eases studying SMMs, it is unclear whether this actually represents shared knowledge structures of a team. This is because the SMM types that are prevalent in a given team, depend on factors related to the task domain, task characteristics and team requirements (BadkeSchaub et al., 2007).

This dissertation proposes that research into SMMs requires an analysis of the knowledge structures that team members hold and the relevance to share this knowledge within the given task context. To study SMMs in ATC the analysis should start broadly and assume multiple separate mental models types as originally suggested (Cannon-Bowers et al., 1993). Moreover, the analysis should also consider an additional mental models type, namely the shared situation model that seems particularly important in ATC and other dynamic environments. Shared situation models represent the understanding of a situation or system response and apply to handling rapidly changing environments (Cooke et al., 2001; Rico, Sánchez-Manzanares, Gil, \& Gibson, 2008; Waller et al., 2004).

Subsequently, the analysis should empirically investigate whether these mental models types adequately represent team members shared knowledge structures or whether a composition of multiple types better describes their shared knowledge structures.

Therefore, this dissertation suggests that in order to understand SMMs in highly complex cognitive environments one first needs to identify the mental models relevant to team members and team performance within the environment. Specifically, this dissertation investigates the question what types of SMMs ATCOs share. This increases the understanding of the mechanisms and dynamics of SMMs in a realistic context and contributes to prevailing framework in SMMs theory. 


\section{The Properties of Shared Mental Models}

SMMs between team members in a team can be both similar and complementary (Mohammed et al., 2010) to enable them to have common expectations (Cannon-Bowers et al., 1993; Mohammed et al., 2010). This property of SMMs is called mental model similarity (Resick et al., 2010). Whether team members have more similar or more complementary mental models depends on the team goals and the task environment. For example, to perform multidisciplinary tasks, effective teams would need specialized roles, functions and complementary knowledge (Cooke, Salas, Kiekel, \& Bell, 2004). In tasks in which team members can interchange roles, highly similar mental models may be more beneficial (Banks \& Millward, 2000). Therefore, SMM theory assumes that knowledge should be similar or compatible, but not identical (e.g. Cannon-Bowers et al., 1993; Rentsch, Small, \& Hanges, 2008; Webber, Chen, Payne, Marsh, \& Zaccaro, 2000). If all knowledge among team members was exactly alike there would be no extra gain, thus no need for a group effort (Badke-Schaub et al., 2007).

SMMs in different task and team contexts should not only be distinguished by their similarity but also by their accuracy. Mental model similarity and accuracy are closely related since conceptually accurate mental models are more likely to be similar (Banks \& Millward, 2007; Edwards, Day, Arthur, \& Bell, 2006). Mental model accuracy refers to the quality of knowledge, often defined as the correctness of rules, concepts and schemas of the real world (Marks, Zaccaro, \& Mathieu, 2000). Mental model accuracy increases with task expertise, so that teams consisting of experts are more likely to hold mental models close to the real world and similar to each other (Edwards et al., 2006). In contrast, teams holding similar mental models do not necessarily have accurate mental models and in that case are unlikely to perform well. This idea becomes clear in the scenario sketched in the beginning. In the scenario, two controllers relieve their colleagues without communicating that an adjacent airport is closed. The fresh-starting controllers therefore miss important information and inaccurately assume that the adjacent airport is open. Thus, although their knowledge is similar (both assume that the adjacent airport is open), it is also inaccurate as the airport is actually closed due to severe weather. This initially leads to inefficiency in the traffic flow because mistakenly cleared aircraft have to be redirected.

Consistent empirical evidence is lacking on the acknowledged role of mental model accuracy, because research has mainly focused on mental model similarity. One reason is that typically in complex task environments multiple accurate mental models exist (Mathieu et al., 2010). In ATC for example, a number of strategies can help to avoid overload on an airport. Controllers could accelerate the amount of aircraft leaving the airport or they could temporary close the airport for inbound traffic. Both strategies are accurate, which makes it difficult to determine a reference model for mental model accuracy (Edwards et al., 2006; Mathieu et al., 2010). Nevertheless, accuracy remains an important property of the SMM construct and research has yet to establish how both properties are exactly related to team performance (Lim \& Klein, 2006). 
The experimental studies of this dissertation address both mental model similarity and accuracy with the aim to reveal a better understanding of these properties and of how they relate to team effectiveness in complex teams. For this purpose, we developed a task environment called TeamTris that allows differentiating between the two properties. In addition, TeamTris allows to index mental model similarity as consistency and not identity between team members' mental models (Smith-Jentsch, Mathieu, \& Kraiger, 2005). A mental model identity index measures the absolute values of agreement between team members to perform a task in a specific way. A mental models consistency index measures agreement of priorities to perform a task in a specific way, but does not measure whether the value of priorities are identical. A mental model consistency index is suitable in task environments in which various solutions results in successful team performance, as it is the case in TeamTris and ATC. For example, ATCOs can adjust the heading or the speed of aircraft to avoid collisions.

\section{Shared Mental Model Development}

Team members' SMMs are believed to develop through the convergence of individual knowledge into shared knowledge structures (Cannon-Bowers et al., 1993; Langan-Fox et al., 2004; Mathieu et al., 2010). This convergence process requires that team members interact with each other and repeatedly exchange information (Kennedy \& McComb, 2010; McComb, 2007). During information exchange, team members often share personally held information and knowledge, which would otherwise remain distributed among individuals.

Distributed information is particularly common in teams with team members that are less familiar with each other. In these teams, deliberate information exchange helps to converge separate mental models of individual team members into SMMs (Cooke, Gorman, Duran, et al., 2007; Gross \& Kluge, 2014; MacMillan, Entin, \& Serfaty, 2004; Uitdewilligen et al., 2010; Waller et al., 2004). As SMMs ease coordination among team members, the mental model convergence process should begin from the start of a team's life cycle (Zijlstra et al., 2012). This helps from the start to reduce uncertainties about for example the responsibilities of other team members so that team members can focus on actual task performance at an early stage (Zellmer-Bruhn, Waller, \& Ancona, 2004).

In this dissertation, it is hypothesized that organizations can help team members to develop SMMs through information exchange during knowledge sharing. Knowledge sharing is the process through which team members exchange relevant knowledge, information and expertise (De Vries \& Van den Hooff, 2006; Mesmer-Magnus \& DeChurch, 2009). More precisely, team members provide and receive explicit and tacit knowledge during knowledge sharing through, for example, communication and observation (Bartol \& Srivastava, 2002). This implies that knowledge sharing can enable the creation of a joint understanding of knowledge that was initially unshared (De Vries \& Van den Hooff, 2006). Consequently, team members will develop more similar and accurate mental models because their individual mental models converge to SMMs. 
Development of shared knowledge becomes a challenge when information exchange between team members is restricted (Stedmon et al., 2007). Such a situation occurs in ATC if team members communicate via datalink. Datalink communication restricts free information exchange as it only allows the communication of pre-defined messages, thus should hamper the development of SMMs (Andres, 2012). Knowledge sharing is specifically interesting in facilitating SMM development when team members communicate via datalink. Therefore, the effect of knowledge sharing on SMMs are studied during verbal communication and during datalink communication.

\section{Team Effectiveness and Team Adaptation}

Team members who have developed SMMs are believed to perform and adapt more effectively. The following sections therefore describe the relationship between SMMs and team outcomes. The section starts with the effects of SMMs on team effectiveness in general and subsequently details into one form of team effectiveness, namely team adaptation.

\section{Team effectiveness}

SMMs have most often been studied with the underlying assumption that mental model similarity and accuracy positively contribute to team effectiveness. Recent meta-analyses strongly support this assumption (DeChurch \& Mesmer-Magnus, 2010a, 2010b). Team effectiveness are represented by a number of outcome variables, such as team performance, decision quality, or safety, and has been assessed in various laboratory and field studies (e.g. Edwards et al., 2006; Gurtner, Tschan, Semmer, \& Nägele, 2007; Kellermanns, Floyd, Pearson, \& Spencer, 2005, 2008; Lim \& Klein, 2006; H. H. McIntyre \& Foti, 2013; Smith-Jentsch et al., 2005).

Although mental model similarity can contribute to team effectiveness, this relationship seems to depend on the type of SMM. Mathieu and colleagues (2010), for example, found that taskrelated mental model similarity revealed a positive linear relationship with team performance, whereas team-related mental model similarity did not. The authors suggest that team-related SMMs take more time to develop and that short-term laboratory studies are inadequate to capture this process. In addition, relationships are more complex than often assumed. Interactive effects of teamrelated and task-related SMM may occur when predicting team effectiveness (Mathieu et al., 2010; Smith-Jentsch et al., 2005). For example, team-related mental model similarity of 306 ATCOs moderated the relationship between task-related mental model similarity and team effectiveness (Mathieu et al., 2010). In other words, the relationship between similar task-related mental models and performance was stronger when team members also had more similar team-related mental models.

Different results have also been found for mental model similarity and accuracy (Banks \& Millward, 2007). Accurate procedural knowledge of student teams positively predicted team performance, but procedural knowledge similarity negatively affected team performance. The authors 
argued that similar procedural knowledge is only important for team members who have similar roles and task. That is, in a task with rather distinctive roles, team members' understanding of each other's responsibilities is less pivotal for performance as each team member individually contributes to the team outcome.

Although this reasoning seems plausible at first sight, several team settings may require similar procedural knowledge although team members have distinct tasks. In the control tower in the example described in the beginning, the start-up controller has different responsibilities than the ground controller. The start-up controller gives clearance to the pilot to start the engines after checking the validity of the flight route. In addition, the start-up controller actively avoids overload on the runways. The ground controller subsequently gives clearances to push back leaving aircraft from the parking position, delegates incoming aircraft to their parking position, and controls aircraft on the taxiway. Although these tasks are different, they are interdependent because the ground controllers cannot do their work without the preliminary activities of the start-up controller. A good understanding of the procedures of either task helps a controller to anticipate and coordinate actions more easily.

The multifaceted findings show the complexity of the SMM construct and emphasize the importance of differentiating between content domains (i.e. task-related and team-related) and properties (i.e. accuracy and similarity) of SMMs. In order to capture the SMM construct as a whole, this dissertation assesses different types of mental models, mental model similarity, and mental model accuracy in relation to team effectiveness.

\section{Team adaptation}

One specific aspect of team effectiveness is team adaptation to novel and unexpected situations. The role of SMMs is particularly interesting in such a situation as this situtaion limits open communication, strategizing and planning and team members must rely on a similar interpretation of environmental cues (LePine, 2005). The described scenario in the beginning of this chapter includes several situations where adaptive team performance is required. For example, ATCOs have to adapt quickly to the new plan to handle the approaching snow cloud and they have to adjust their planning when the adjacent airport is closed and additional aircraft need landing clearances. Although these are relatively routine changes, team members may only apply the same strategies and task procedures if they have a similar understanding of the situation.

SMMs thus enable team members to adapt effectively by providing a common and structured understanding of the new situation and facilitating the development of a common strategy (Burke et al., 2006). Although it makes sense that team members must adapt their mental models in terms of both accuracy and similarly, Marks and colleagues (2000) only found a significant relationship between mental model similarity and team adaptation. It seems that initial mental model accuracy is less important for adaptive team performance. The authors infer that similar mental models may in 
turn facilitate the development of accurate mental models in novel situations. However, they also indicate that this interpretation may only be true under certain conditions, e.g. for inexperienced teams in their early developmental phases with immature SMMs (Marks et al., 2000). But also experienced team members may still be inexperienced on tasks that rarely occur, such as emergency situations in ATC (Malakis, Kontogiannis, \& Kirwan, 2010a; Malakis et al., 2010b). It is possible, that these teams have inaccurate although similar mental models when they encounter a unique situation.

The positive effect of both mental model similarity and accuracy on team adaptation was demonstrated for a less dynamic and complex task in a laboratory experiment (Randall, Resick, \& DeChurch, 2011). Project student teams participated in decision-making task on city management that first required managing the growth of a new city and then suddenly the revitalization of a developed city. Project teams with highly similar and accurate mental models about task strategy easily adapted their strategies. They also made more effective decisions in novel situations than teams with less similar and accurate mental models. Mental model accuracy contributed to adaptive team performance by helping team members to generate and evaluate the effectiveness of possible strategies in new situations. Mental model similarity enables team members to agree on one strategy and coordinate the implementation (Randall et al., 2011).

These findings support the cyclic model of team adaptation (Burke et al., 2006) that emphasizes the importance of SMMs in three subsequent phases of the adaptive process. This means that SMMs help team members to assess the new situation in a similar way, formulate a common plan of how to approach the new situation and coordinate the execution of the plan successfully. In addition, the interactive process between team members when adapting to a change further reinforces their SMM. This close relationship between SMMs and team adaptation implies that adaptive team performance is not possible in the absence of SMMs (Burke et al., 2006). Team members adapt proactively when they have a similar understanding of the equipment, the task and the roles and responsibilities of other team members.

Despite the suggested positive effects of SMMs on team adaptation, there are also some concerns. In profoundly changing situations mental models may tend to alter too slow and rest upon incomplete false information (Uitdewilligen et al., 2010). This may result in a mismatch between mental models and the environment and a decrease of mental model accuracy. Any delay in successful knowledge updates may consequently lead to a performance drop (Weick, 1990). This may hold true especially for initially successful teams who suddenly face a rapid change in the environment. These teams have developed stable and effective SMMs over a prolonged period of time and this actually hampers them from quickly adapting to a sudden unforeseen change (Audia, Locke, $\&$ Smith, 2000). This thought is in line with and extends the established idea from cognitive psychology that automated behaviour is hard to change (Sternberg, 2008).

In contrast to ideas of the cyclic model of team adaptation (Burke et al., 2006), Uitdewilligen and colleagues (2010) argue that SMMs may be detrimental in the first two phases of the adaptive 
process. First, during situation assessment (phase 1), the false but common perspective and similar interpretation of cues may make team members inattentive of the actual changes in the environment. Second, during plan formulation (phase 2) creative processing may be hampered through similar perspectives leading to less new and diverse ideas (e.g. Jehn, Northcraft, \& Neale, 1999).

In sum, two notions exists on the role of SMMs regarding a team's ability to adapt to heavily changing situations and it is interesting to further investigate these two notions. An investigation is also essential to work environments such as ATC because novel, unforeseen and unpredictable situations frequently occur and it is desirable that team members adapt adequately and quickly. The current research tests whether mental model accuracy and similarity are influential in adaptive performance and thereby contributes to the literature of team adaptation.

\section{Communication as Indicator and Facilitator of Shared Mental Models}

Communication is an indicator of SMMs as team members with effective SMMs communicate more effectively. Communication analysis therefore offers an indirect and additional measure of SMMs that is appraised during task execution. During task execution effective communication becomes apparent in a number of team behaviours (Van Avermaete \& Kruijsen, 1998). Three team behaviours are therefore associated with the presence of SMMs and lead to enhanced team performance (Salas, Rosen, et al., 2007).

The first team behaviour associated with SMMs is closed-loop communication. This is a sender-receiver pattern in which the information that is transferred by the sender is acknowledged and understood by the receiver and is finally cross-checked by the sender again (R. M. McIntyre \& Salas, 1995). Effective communication during this process is concise, standardized and implicit especially in high workload situations. The second team behaviour associated with SMMs is mutual performance monitoring, which is the awareness of the performance and workload of other team members. By noticing other's behaviour and level of stress, relevant information can be offered in a timely and effective manner, even before it is requested. The scenario in the beginning of this chapter describes an illustrative example of mutual performance monitoring. When the assistant controller realises that the supervisor misses relevant information regarding the procedures of the landing of an A380 aircraft, the assistant immediately looks up the information in the manual and provides the information to the supervisor without request. The third team behaviour is adaptive and supportive behaviour, which comprises helping out team members in difficult situations or perceiving abnormalities in other's behaviour. This behaviour enables team members to disperse workload dynamically and creates an open atmosphere to offer and ask for assistance (Porter et al., 2003).

SMMs not only affect team behaviours but, in turn, are influenced by these behaviours (Marks, Mathieu, \& Zaccaro, 2001). In other words, SMMs are reciprocally correlated to team behaviours (DeChurch \& Mesmer-Magnus, 2010b) and specifically communication contributes to the emergence and maintenance of shared knowledge (Burke et al., 2006). As discussed earlier, 


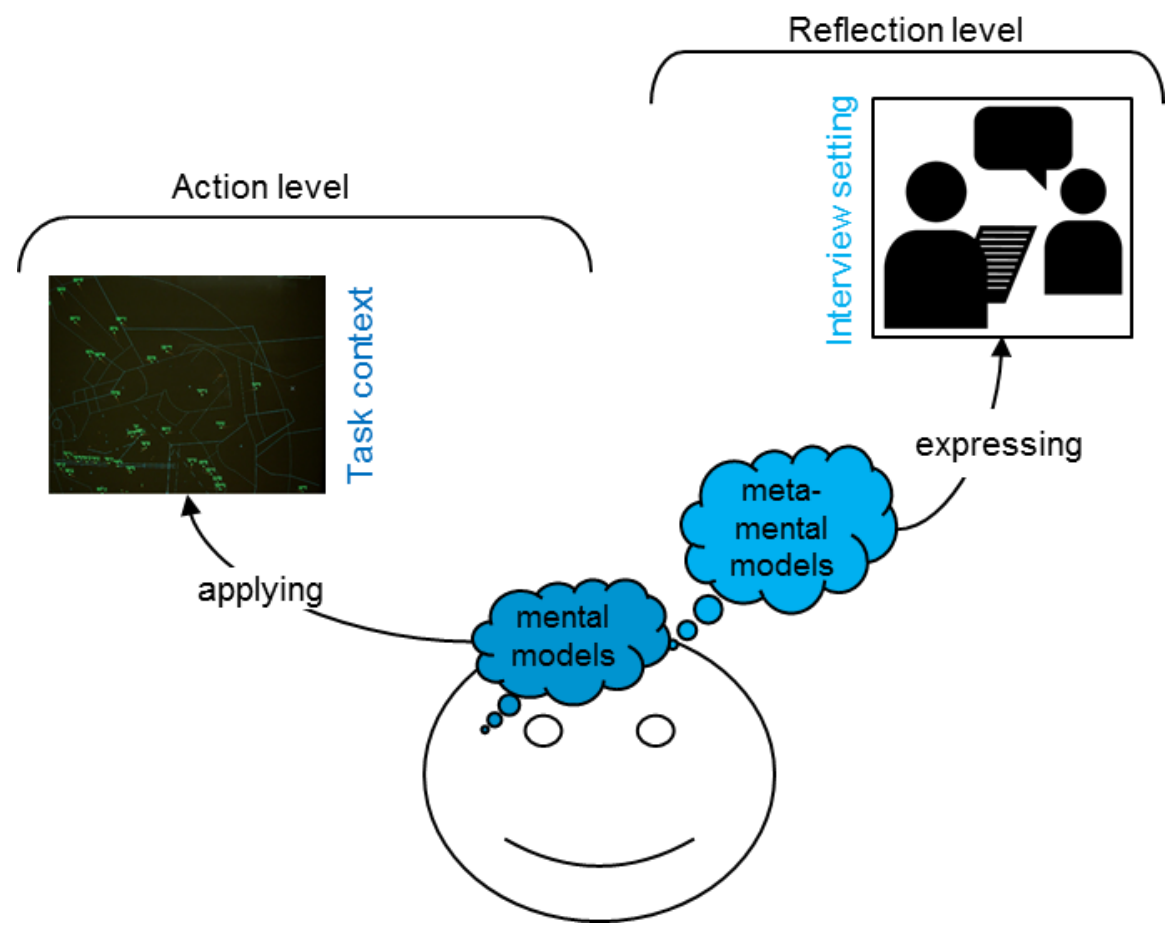

Figure 1.1. The Two-Level Approach of SMMs

information exchange between team members of newly formed teams helps to align individual mental models (Zijlstra et al., 2012). During this mental model convergence process overt communication is crucial in reaching cognitive consensus (Andres, 2012; McComb, 2007). Further study is required to understand this apparent positive effect of communication on SMMs that only a few studies have examined previously (e.g. Jo, 2012; MacMillan et al., 2004; Wilson, Salas, Priest, \& Andrews, 2007). Therefore, communication plays an essential role in this dissertation. Communication is used as an indicator of team behaviours associated with SMMs. In addition, the direct relationship between communication and SMM development is investigated. Particularly, it is tested whether communication between team members contributes to SMM development.

\section{The Two-Level Approach of Shared Mental Models}

This section introduces the Two-Level Approach of SMMs, which clarifies how the concept of SMMs is approached in the subsequent studies of this dissertation. Figure 1.1 illustrates the model including the main idea is that studying the reflection on knowledge by experts reveals different results than studying the use of knowledge in a dynamic task setting. The model is based on ideas of declarative versus procedural knowledge (Anderson, 1981) and on observations and conversations with ATCOs in the field.

The various circumstances in which ATCOs apply their knowledge are innumerable because every traffic situation is unique due to the influence of numerous factors, such as weather, capacity limitation and aircraft performance. These complex situations are inherent to ATC, but applies also to other dynamic work environments. In these often quickly changing environments, experts use their 
experience to quickly apply knowledge under different circumstances (Oprins, Burggraaff, \& Van Weerdenburg, 2006). This quick use of knowledge is possible because they have internalised their knowledge and use many knowledge aspects intuitively and automatically (Hacker, 2003; Salas, Cannon Bowers, \& Johnston, 1997). This type of knowledge is also referred to as tacit or procedural knowledge, which is typically not easy to articulate (Anderson, 1981). Consequently, if individuals are asked to describe their knowledge structures they may probably refer to declarative knowledge that is readily accessible rather than being able to describe the tacit knowledge that they actually apply during task execution.

In order to account for the discrepancy between formalised declarative knowledge and tacit procedural knowledge, I put forward the Two-Level Approach of SMMs. According to this approach, SMMs must be examined at two different levels: (1) the reflection level and (2) the action level. The reflection level takes the broad picture of a profession into account. It addresses declarative knowledge that has formally been learned, and gives an impression of what an individual believes is important knowledge to execute the task or work together in a team. The reflection level follows the idea of metacognition (Hinsz, 2004) as it comprises 'knowledge about knowledge'. This metaknowledge, or meta-mental model, can be assessed by asking people to describe their profession and what they communicate to colleagues, and explain what knowledge they need to perform their work (e.g. in an interview setting). This type of language use is also present in job descriptions and procedural manuals that are therefore a basis to understand SMMs on the reflection level.

Knowledge on the action level is situation specific and sensitive to the task context and the individuals involved. This level captures how an individual actually applies procedural knowledge in multiple circumstances depending on both external and internal factors. This type of knowledge is often difficult to verbalize and make explicit. In order to get access to the mental models on the action level, scenario-based elicitation of knowledge is required, to include a realistic task context.

The idea that actions can be purposefully carried out with little awareness of the required knowledge emerged in the early 70's (Hoffman, Shadbolt, Burton, \& Klein, 1995). Team processes such as communication and coordination are the key elements to investigate applied knowledge and can best be assessed via observations rather than by reports (Cooke, Gorman, Duran, et al., 2007). The notion of the action level corresponds with the view that knowledge elements are used dynamically depending on situational parameters (Cannon-Bowers et al., 1993). Hacker's Action Theory (1978, 1986) rests upon similar ideas. Hacker differentiates between "Aufgabe" ${ }^{3}$ - what ought to be done/ what is said to be done - and "Handlung" - what is done. Knowledge used to describe a Aufgabe or Handlung may be very different varying by situation and person because knowledge is often context specific depending on goals, situational cues or partners (Hacker, 2003).

\footnotetext{
3 "Aufgabe" can be translated with "task"

4 "Handlung" can be translated with "activity"
} 
Most of the research done into SMMs focuses on the reflection level and rarely investigates SMMs during task execution or with regard to specific controlled situations. For example, in studies that apply pair-wise comparison ratings, team members evaluate the similarity of words that are presented without a context. The similarity of these ratings among team members is used as an index of their SMMs. Although such methods led to a substantial body of theoretical knowledge of SMMs, additional research is required to study how team members actually apply their knowledge during task execution. "Implicit, unarticulated pieces of knowledge [...] cannot be elicited by questioning alone" (Hacker, 2003, p. 115), and therefore, measurement of the action level requires on-task methods, such as the appraisal of behavioural markers, communication analysis, and scenario-based assessment.

The Two-Level Approach of SMMs determines how SMMs are appraised in the various studies in this dissertation. These studies address both the reflection and action level to reveal conceptual and applied aspects of SMMs. The following section describes the research aims and gives an outline of the studies reported in this dissertation.

\section{Research Aims and Outline of the Dissertation}

The overall purpose of this dissertation is to increase the understanding of SMMs in complex teams and specifically teams of ATCOs. This dissertation studies what types of mental models are relevant in ATC and how SMMs may contribute to safe and efficient air transportation. Thus, it specifically focuses on teams in ATC, but where possible it results are also generalized to other complex teams.

This dissertation has three research aims with related research questions. The first aim is to refine the SMM construct in an operational context, namely in the domain of ATC. $\mathrm{We}^{5}$ study what knowledge ATCOs share to perform effectively as a team and test whether exiting typologies of SMMs (Cannon-Bowers et al., 1993; Mathieu et al., 2005) adequately describe the types of SMMs relevant in ATC. This investigation must help to clarify whether conceptual ideas on the types of SMMs found in the literature apply to specific and complex teams in the field. In addition, this investigation attempts to contribute to the understanding of teamwork between ATCOs through studying their SMMs.

The second research aim is to reveal the role of knowledge sharing in developing SMMs. Specifically, we question whether guiding team members in knowledge sharing helps them to develop more similar and accurate mental models. To answer this question, we combine the literature on mental model convergence (McComb, 2007) and knowledge sharing (e.g. Wang \& Noe, 2010). This increases our understanding on how the process of mutually exchanging information contributes to the convergence of individual mental models into SMMs. Moreover, this expands knowledge about how organizations can support team members in SMM development and eventually improve team performance

\footnotetext{
${ }^{5}$ The studies described in this dissertation were carried out with several co-authors and I use 'we' when I refer to the co-authors and myself.
} 
Finally, the third research aim focuses on investigating the relationship between SMMs, communication and adaptive team performance in response to an unforeseen event. We study the question if SMMs and communication positively affect team members' ability to adapt to a substantial change in the task environment. To answer this question, we test aspects of a conceptual model of team adaptation (Burke et al., 2006) and we test whether phases of adaptive behaviour found in individuals also apply to teams (Lang \& Bliese, 2009). This dissertation consists of five chapters that address the research aims presented and approach SMMs on both the reflection and action level.

Chapter 2 considers the first research aim, refining the SMM construct in an operational context. The chapter describes ATCOs' mental models, derived from a cognitive task analysis. The analysis establishes the structure and functionality of shared knowledge, and considers the contexts in which experts apply knowledge. This results in the presentation of a domain-specific framework of SMMs. The framework describes the knowledge structures that individual ATCOs should possess and teams of controllers should share. It establishes the conceptual basis for the subsequent studies of this dissertation. Chapter 2 approaches knowledge on the reflection level as the results are primarily based on interviews and observations.

Chapter 3 builds on the SMM framework developed in this first work and further elaborates on the first research aim. We carry out a card sorting study to investigate the distinct validity of the different mental model types identified in the cognitive task analysis. We analyse the data of 100 ATCOs by means of multidimensional scaling and permutation tests. This then allows the comparison of mental model similarity of ATCOs of two different air navigation service providers, as well as with different levels of expertise. Although a card sorting task captures some unconscious relationships of knowledge elements, this study approaches SMMs on the reflection level since the elicitation is independent of specific situations.

Chapter 4 addresses the second research aim, revealing the role of knowledge sharing in developing SMMs. In this study, student teams are guided to share task-related and team-related knowledge prior to task performance. We then investigate whether guided knowledge sharing facilitates the development of SMMs and whether SMMs affect team effectiveness. We also assess if knowledge sharing increases communication among team members and whether this further improves their SMMs. Chapter 4 introduces the task paradigm used in the experimental studies of this dissertation, called TeamTris (Van der Pal \& Justen, 2011). We developed TeamTris specifically to simulate the cognitive processes and tasks of ATCOs and other dynamic teams. The task is simple enough to be performed by unexperienced participants and allows SMMs to be studied during task performance. The studies presented in Chapter 4 - Chapter 6 use TeamTris, which allows SMMs to be assessed on the action level as we can observe and measure how team members apply their knowledge.

Chapter 5 discusses a specific aspect of the second research aim, namely the impact of knowledge sharing on the development of SMMs and on associated team behaviours during datalink 
Chapter 1

communication. Datalink is a communication technology, which is becoming increasingly available in ATC. The study presented assumes that information exchange in datalink environments is limited to pre-defined messages and thus hampers the development of SMMs. We test the hypotheses that providing additional opportunities of guided knowledge sharing between task trials stimulates the development of team members' SMMs, closed-loop communication and backing-up behaviour. This should in turn positively affect team performance. As the study looks at teams communicating via datalink, it is particularly interesting to ATC but also gives insights into the development of SMMs in other complex teams working in technology-driven communication environments.

Chapter 6 considers the third research aim - investigating the relationship between SMMs and adaptive team performance after an unforeseen event. We combine the literature on adaptation and SMMs, and examine the role of team members' shared knowledge ${ }^{6}$ and standardised communication protocols in adaptive team performance. Particularly, we assume that mental model similarity, mental model accuracy and standardized communication help teams to adapt effectively to a change. We test these hypotheses by studying student teams who perform TeamTris and experience an unexpected partial system breakdown after three gaming trials. By applying discontinuous growth modelling, we can assess and visualise differences between teams during the two phases of adaptation, which are transition adaptation and reacquisition adaptation.

Chapter 7 establishes the overall conclusion of this dissertation and discusses the main findings of the different studies. It provides an overview of the theoretical and practical implications and discusses the possibilities for future research.

\footnotetext{
${ }^{6}$ Note, that primarily the term 'shared knowledge' is used in this chapter because it is a more common in the adaptation literature. However, 'shared knowledge' is interchangeable with the term 'shared mental models'.
} 


\title{
Chapter 2
}

\section{Identifying shared mental models within teams of air traffic controllers: A cognitive task analysis}

\begin{abstract}
Notions about shared knowledge suggest that team members share multiple types of mental models and the present study tests this theoretical assumption in the domain of air traffic control. This is important because shared mental models contribute to efficient teamwork but the often abstract nature of the construct prevents its successful application in the field. We identified knowledge elements that are applied and shared by air traffic controllers through a cognitive task analysis comprising three methods of qualitative data collection. First, preliminary conceptual modelling served to build an initial and general structure of the air traffic control task. This contributed to the next two research steps, which elicited shared knowledge among air traffic controllers through interviews and observations. Data from these investigations yielded a framework for shared mental models in air traffic control teams. This study shows that controllers should possess and share knowledge pertaining to the equipment, the task, the team, team interaction and the situation. It is discusses how this insight differs from existing typologies and how it can contribute to training design and team performance assessment in air traffic control.
\end{abstract}





\section{Introduction}

Air traffic controllers (ATCOs) usually cooperate in teams as their work is too demanding to permit individual tasks to be effective or safe (Malakis et al., 2010b; Mathieu et al., 2010). Successful team performance is only possible when task roles and knowledge are both dispersed and shared among controllers (Cannon-Bowers et al., 1993; Fiore et al., 2010). Dispersed knowledge supports the different task roles that the individual team members need to fulfil, and shared knowledge enables efficient interaction among team members to lead to effective team performance (e.g. Cannon-Bowers et al., 1993; Smith-Jentsch et al., 2005). Through a better understanding of ATCOs' knowledge structures it may therefore be possible to facilitate teamwork.

A general notion to understand controllers' knowledge structures is the construct of shared mental models (SMMs). SMMs are mental representations of task- and team-relevant aspects that are shared among team members (Mohammed \& Dumville, 2001; Sander, van Doorn, van der Pal, \& Zijlstra, 2015). However, this construct needs refining in air traffic control (ATC) since it rarely describes teamwork in context, thus has only limited applied relevance (Smith-Jentsch et al., 2005). The aim of the present study therefore is to appraise the details of shared knowledge structures of ATCOs. Such a study is important because ATC teams need to coordinate seamlessly and perform efficiently, and both training and expert performance may gain from a better understanding of controllers' SMMs (Malakis et al., 2010b; Mathieu et al., 2010).

The present study investigates three research questions that will be answered on the basis of a cognitive task analysis composed of on a number of applied qualitative data collection methods (Militello \& Hutton, 1998; Wei \& Salvendy, 2004; Zachary, Ryder, \& Hicinbothom, 1998). These methods will identify and chart the types of knowledge that an ATCO needs and shares with fellow team members. This will in fact provide insights into the concrete behaviours, knowledge and team processes that team members should possess, and thus provide a detailed overview of SMMs in ATC teams (Mohammed, et al., 2010). Such a cognitive approach should enhance the practical relevance of the construct of SMM and should lead to tailored recommendations to improve teamwork training in ATC.

The next section first describes briefly the background of SMM theory and formulates the specific research questions addressed in this study.

\section{Shared Mental Model Typologies}

SMMs are knowledge structures of team members that enable them to interpret and explain the task and each other's behaviour in a similar and accurate manner (Cannon-Bowers \& Salas, 2001). It is believed that multiple types of mental models can be distinguished (Mohammed et al., 2010). A widely accepted typology of SMMs suggests four distinctive types of mental models (Cannon-Bowers et al., 1993) and these may also be applicable to ATC teams. Accordingly, team members should have 
Chapter 2

a good understanding of the system and equipment they interact with (equipment model). Second, they must share knowledge about their joint task and the job they accomplish (task model). This means that they should have a common understanding about task procedures, rules, and strategies and interpret environmental constraints regarding task execution in a similar way. A third model pertains to mutual understanding of the competencies of fellow team members and how a member's own task contribution affects the performance of the other members (team model). Finally, team members should have the same knowledge about how to interact with each other and understand each other's information needs adequately (team interaction mental model).

These four types of SMMs are often trimmed into two knowledge domains in studies of team knowledge of ATCOs, namely to a task-related mental model and a team-related mental model type (Mathieu et al., 2000). The first pertains to knowledge about the equipment and the task, while the latter entails knowledge about the team and team interaction. This distinction has been adopted by many researchers.

However, neither the first typology nor the more condensed typology has been adequately evaluated in the field, which often restrains practitioners to use the concept to improve team performance. A systematic study into SMMs in the field, here ATC, should enrich the theoretical understanding of the concept and provide detail to the separate types of SMMs (Mohammed et al., 2010; Smith-Jentsch et al., 2001).

\section{Research Questions}

To investigate SMMs in ATC, this study focusses on three research questions that each addresses a separate aspect of shared knowledge assumed in SMM theory, namely the structure of knowledge, the functionality of knowledge sharing among team members and the role of the context on shared knowledge (Cannon-Bowers et al., 1993; DeChurch \& Mesmer-Magnus, 2010b). By studying the interrelationship of individual knowledge structures, i.e. explicate the relationship between team cognitive elements (DeChurch \& Mesmer-Magnus, 2010b), we address both their content and organisation (Kraiger \& Wenzel, 1997) and are able to draw conclusions on the various mental model types that exist in ATC. The first research question therefore is: How is the individual knowledge of ATCOs organized in separate types of mental models?

The second research question deals with the functional relevance of sharing separate types of mental models in specific team contexts. Notions on SMMs suggest that shared knowledge within a team is likely to be task-dependent (Cannon-Bowers et al., 1993). For example, team members who work in highly automated environments presumably benefit from a similar understanding of system functioning and their interaction with the system. On the other hand, team members who directly interact with each other, rather than via a system, may work more efficiently if they have a similar understanding of their information needs. This means that the characteristics of the task environment 
determine whether sharing specific knowledge is functional. The second research question therefore is: What is the functional relevance of sharing separate types of mental models in ATC?

The third research question addresses the context (or situation) and refers to whether shared knowledge is applicable in a number of situations that ATC teams are commonly exposed to (CannonBowers et al., 1993; Kozlowski, Watola, Jensen, Kim, \& Botero, 2009). They often face unusual occurrences and situations that are associated with uncertainties (Malakis et al., 2010a). Such unique situations may require different combinations of mental models to apply existing knowledge in a flexible way. This means that this final research issue requires investigating the influence of the context on individual or SMMs. The third research question is: How do the identified mental model types in ATC apply in various situations?

The research questions will be answered on the basis of a cognitive task analysis consisting of three subsequent qualitative data collection methods. The incremental findings from these methods will be collated into a structured overview that identifies the most prevalent and shared knowledge elements in ATC teams. The next section first describes the difficulties in eliciting (shared) knowledge structures in teams that operate in dynamic task environments and how cognitive task analysis can help to overcome these difficulties.

\section{Cognitive Task Analysis to Elicit Shared Knowledge in ATC Teams}

Gaining access to shared knowledge in ATC teams is difficult because these teams perform in a highly complex and dynamic environment in which the use of knowledge changes rapidly (Malakis et al., 2010b). The present study approaches these difficulties by applying a cognitive task analysis (CTA), which enables the understanding of the cognitive requirements of individuals who interact in complex environments (Crandall, Klein, \& Hoffman, 2006). CTA is often applied to provide a systematic cognitive overview of knowledge structures, processes and strategies as they occur in natural settings (Craig et al., 2013; Klein \& Militello, 2001; Mohammed et al., 2010; Seamster, Redding, \& Kaempf, 1997). The method has been applied successfully in various dynamic environments including aviation (Hess, MacMillan, Serfaty, \& Elliot, 1999; Niessen, Leuchter, \& Eyferth, 1998; Seamster, Redding, Cannon, Ryder, \& Purcell, 1993).

CTA mostly employs one or more data collection techniques to obtain knowledge from operational experts (Schraagen, Chipman, \& Shalin, 2000). In fact, a combination of techniques (mixed methods) is recommended to increase the validity of the results (Crandall et al., 2006). The present study applies three methods in a stepwise manner, namely preliminary conceptual modelling of the ATC task, semi-structured interviews and lastly observations. This mixed-method approach is meant to compare existing notions of SMMs (Cannon-Bowers et al., 1993; Mathieu et al., 2000; Mohammed et al., 2010) with the knowledge structures that are actually shared among ATCOs. Such a comparison should both demonstrate how and when sharing knowledge in ATC is functional and add more detail to SMM theory. 


\section{Data collection}

\section{Preliminary Conceptual Modelling: An initial overview of the ATC Task}

The first step was to create an initial overview of the ATC task including the illustration of the relevance of knowledge in the ATC field. The aim was to gain a thorough understanding of both the composition of task elements and the process of how individual knowledge structures evolve into shared knowledge structures among team members. This helped to understand the role of SMMs in the overall ATC task and formed the basis for subsequent data collection by giving directions towards the aspects that needed to be addressed during the analysis.

The International Civil Aviation Organization (ICAO) defines ATC as follows: “.... a service provided for the purpose of preventing collision between aircraft, and on the manoeuvring area between aircraft and obstructions; and expediting and maintaining an orderly flow of air traffic." (ICAO, 1996, pp. I-3) This definition captures the main objective of all ATCOs, which should motivate and regulate their work. It includes continuous monitoring and evaluating of aircraft behaviour that are necessary to identify potential safety issues or bottlenecks in air traffic. An example of such a safety issue may be the deviating behaviour of a specific aircraft in terms of distance toward other aircraft.

The overall objective of maintaining safe air traffic breaks down into specific goals, such as to keep aircraft physically separated from each other (Malakis et al., 2010a). This necessarily results into smaller sub-goals, such as adjusting the planning to ensure safe separation. A sub-goal in turn initiates concrete tasks or activities, such as redirecting the aircraft or to increase aircraft speed. This representation of the complex task environment of an ATCO described agrees well with Hacker's cognitive oriented action-regulation theory (Hacker, 2003) that distinguishes several levels of goals in a task environment. Moreover, it is corroborated by the empirical literature that successful execution of (sub-)tasks requires that individuals show specific competencies and demonstrate knowledge, skills and attitudes (Abma, Van Bavelgem, Cromie, Liston, \& Ward, 2004).

Generally speaking, the required knowledge is believed to be structured and organized in mental models that describe, explain, and predict the behaviour of the system that a controller is working with (Rouse \& Morris, 1986). Competencies such as communication and coordination help ATCOs to share these mental models with fellow team members, thus contribute to the developments of SMMs (Kennedy \& McComb, 2010; Langan-Fox, Sankey, \& Canty, 2009; Mohammed et al., 2010). A particular interest of the present study is therefore the communication and coordination processes between controllers. 


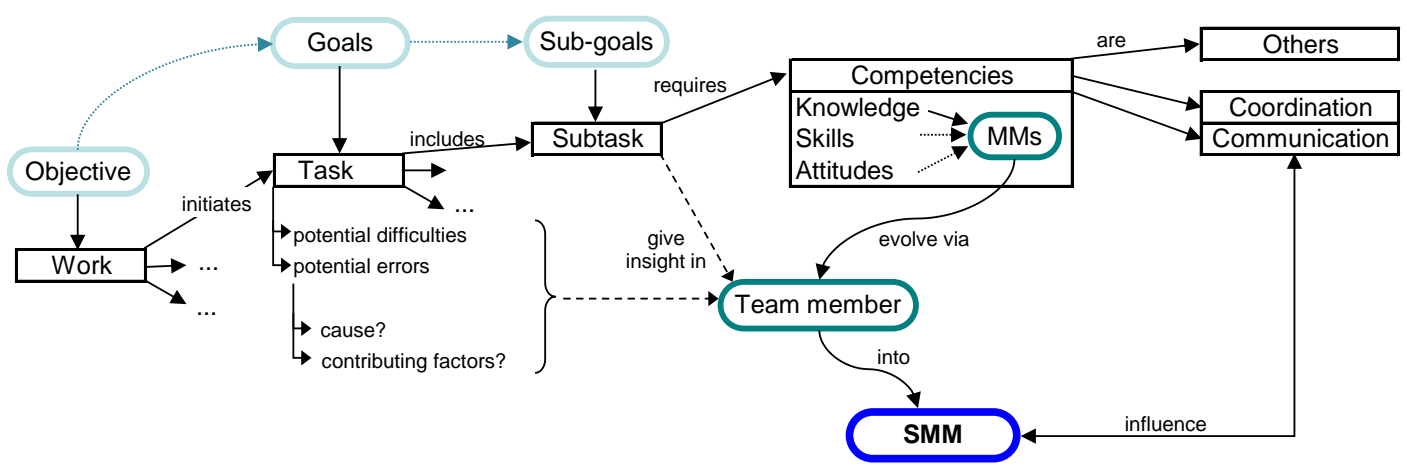

Figure 2.1. Graphical presentation team member's position within a work environment. The representation distinguishes levels of goals, the individual competencies and the possible relationships with other team members.

Figure 2.1 summarizes in a conceptual model the described competencies of individual ATCOs in light of the main goals they face. The figure also shows that individual mental models of team members may converge into shared knowledge structures. The different elements depicted in Figure 2.1 are directly or indirectly related to knowledge structures of team members and form the starting point of a more complete analysis of SMMs in ATC.

The subsequent interviews and observations were carried out to both follow up on and specify the overview so far. These data collection methods were meant to access shared knowledge that is specific to the task and to the context in which ATC task takes place (Schraagen et al., 2000).

\section{Semi-Structured Interviews}

The second data collection method comprised a number of semi-structured expert interviews. This method was meant to find answers to more specific questions that emerged from the modelling study phase. A number of interview sessions therefore applied a thematic structure of predefined questions, but also allowed flexible elaboration on other topics that the expert participants found relevant (Crandall et al., 2006; Lindlof \& Taylor, 2011). The results aimed to give a detailed overview of the knowledge structures of the ATCOs as individuals but also as part of a team. For this purpose, the interviews included an assessment of controllers competencies as these pertain to knowledge, skills and attitudes (Abma et al., 2004). In order to understand when and why knowledge is applied, interviews aimed to reveal the actual tasks and goals that a team of ATCOs faces, and how these tasks are triggered in different circumstances.

Participants and procedure. Participants of the interviews were all expert ATCOs but varied in the number of years of work experience. Since not all participants were comfortable with data recording, two researchers took notes while one researcher guided the interview.

In total, three interview sessions were conducted at the Dutch Air Traffic Control. The first three-hour session was a focus group interview with one active controller and two recently retired 
controllers with experience as radar controller, supervisor and operational expert. All were male and had an average working experience of 25 years $(S D=10.69)$. The focus group interview allowed discussion among interviewees, which enabled an immediate comparison between various perspectives on for example, effective (team) task strategies or the impact of trust on team functioning (cf. Schraagen et al., 2000). In other words, the approach was selected to increase the understanding on the aspects controllers strongly agreed on and also on those aspects that were subject to interpretation as based on individual opinions and attitudes.

Two additional individual interviews of two hours were conducted with two male controllers. One was a radar controller with one year of work experience as both planner and radar controller. The other had 13 years of experience including six years of experience as a supervisor. This latter interview focused exclusively on the requirements of the supervision function and thus offered an additional perspective. Both interviewees were male and each session took about two hours.

Interview scheme. All interviews were performed according to a predefined interview scheme. The interview scheme (Appendix II.I) was developed on the basis of the overview that resulted from the preliminary conceptual modelling (Figure 2.1). Each interview addressed six aspects that captured individual knowledge, shared knowledge and task context according to the earlier formulated research questions.

The first three aspects covered individual task and equipment knowledge and were approached via a scenario-based interview technique that has been applied in a range of aviationrelated studies (e.g. Van der Pal, 2005). This technique aimed to reveal authentic aspects of operational conditions and competencies that go beyond the basic skills, knowledge levels and formal tasking. For that purpose, the three operational experts that were part of the interview panel were encouraged to consider the ATC task from a number of perspectives (e.g. planner or radar controller). They answered questions about both routine and non-routine working days, and were asked to describe the situations that make ATC tasks particularly difficult or complex.

The remaining three aspects covered by the interview scheme had a direct relevance to teamwork and shared cognition. The aim was to reveal team competencies, interrelation of different team members, and how, when and what knowledge controllers need to share.

\section{Observations}

The third data collection method included a number of observations of ATCOs at work and was meant to focus primarily on knowledge application and knowledge sharing during task performance (Crandall et al., 2006). The goal was to determine specific knowledge requirements on task procedures and potential automatic processes within a team. These included interactions with the workstation and with other team members, but also pertained to how ATCOs handle the (changing) environmental constraints in which they have to operate. These cognitive aspects were assumed to become manifest in observed behaviour, such as communication, coordination, and teamwork 
behaviour (cf. McComb, 2007). Communication was evaluated on four separate aspects: (a) message content and timing, (b) the communication medium, e.g. radio, telephone or datalink messaging, (c) the type of communication, i.e. verbal or non-verbal, and (d) the individuals involved, i.e. sender and receiver.

On-Site observations. Overall, six controllers, different from the ones who were interviewed, were observed for a total of 10 hours. The observation sessions took place at an Area Control Centre and focused on three main functions: the planner controller, the radar controller and the supervisor. Data was collected during daily shifts including peak hours and while no unusual events occurred. Some restricted interaction between observers and the observed controllers was possible without interrupting the natural flow of activities, as controllers often spontaneously verbalized their activities and decisions.

Observations of crisis management training. An additional observation took place during two 45 minute sessions of crisis management training of ATC personnel. The purpose was to study knowledge application and sharing in several high workload situations (Waller et al., 2004) in which controllers had to face unforeseen situations. Each 45-minute training session simulated several non-routine events, such as loss of contact with an aircraft, hijack, and a crash at an adjacent airport. A team consisted of a supervisor, the flow management position, four area controllers and two controller assistants. The observation focused on information exchange, teamwork behaviour and coordinating activities.

\section{Coding Criteria}

To account for the thematic approach via research questions, the notes taken during interviews and observations were transcribed and subsequently reviewed by the participating controllers on accuracy and completeness. Two coding schemes were used to organize the data (Auerbach \& Silverstein, 2003; Schraagen et al., 2000). The coding schemes were based both on the research question and SMM theory, but also partly emerged from the data at hand (Auerbach \& Silverstein, 2003). This approach was applied to ensure a broad coverage of knowledge aspects pertaining to individual and shared knowledge structures. This means that a first coding scheme helped to structure the data according to the research questions and distinguished whether data pertained to knowledge structures (first research question), the functionality of sharing knowledge (second research question), or the dynamic application of knowledge (third research question). For example, when the data contained elements like "sharing", "being on the same page" or "similar understanding" these data elements were coded as belonging to the second research question. Also, when controllers mentioned the context or the situation as an influential factor of how they behave and apply knowledge theses data elements were marked as pertaining to the third research question.

The second coding scheme aimed to identify the separate mental model types in ATC. Based on the notion of SMMs described we assumed four general categories of mental models, namely 
Chapter 2

equipment, task, team and team interaction mental models (Cannon-Bowers et al., 1993). For example, data that was related to the automated system (e.g. description of the radar screen) was categorized as equipment knowledge and data that described the collaboration among controllers (e.g. cross-checking information) was allocated to the team interaction category. Information that was neither related to equipment, task, team, or team interaction mental models was initially coded "not otherwise specified". This information was often situation dependent and could pertain to the location of an aircraft or described weather forecasts. These knowledge elements formed a new mental model type, namely the situational mental model.

\section{Results}

While data collection through interviews and observations took place at subsequent moments in time results are integrated and presented according to the three research questions. First, the identified types of mental models and their functionality to share theses are described (1st and 2nd research questions). Second, the application of shared knowledge in varying and changing situations is outlined (3rd research question).

\section{Types of Mental Models and the Functionality of Sharing}

This section reports on the key findings from the interviews and observations to answer the first two research questions. These findings therefore cover the structural organisation of knowledge in mental model types and the functional relevance of sharing these types in the ATC task environment. We identified five prevalent mental model types that are represented by an individual ATCO, namely the (1) equipment, (2) task, (3) team interaction, (4) team, and (5) situational mental model. Per mental model type the required knowledge content and practical examples are specified in the text and in the Table 2.1 to Table 2.5.

The equipment mental model. Table 2.1 provides a concise overview of the findings regarding a controller's knowledge of equipment. Three general knowledge elements basically pertain to this knowledge type.

ATC specific systems. The interviews and observations showed that a team of ATCOs depends heavily on technical equipment, such as radar, radio, flight information and planning software. Controllers use regularly updated system information of the current and expected traffic situations in the near future to form an accurate mental picture of the airspace. The algorithms of these automated systems are not necessarily transparent, but controllers reported a good understanding of the data provided by the system. Their system understanding is sufficient for subsequent decision making. Even though ATCOs are heavily supported by the system, they remain in control of making the final decisions.

Role of the systems. Interviewed controllers stated that they perceive the equipment as indispensable for their work, but also believe that it is not directly related to teamwork. This finding 
Table 2.1

The Equipment Mental Model

\begin{tabular}{ll}
\hline Definition: knowledge about role and limitations of the equipment \\
\hline ATC specific systems & $\begin{array}{l}\text { ATC specific systems, such as radar system, the radio, and flight data } \\
\text { processing }\end{array}$ \\
$-\quad \begin{array}{l}\text { provide information of the past, the present and future states of } \\
\text { aircraft }\end{array}$ \\
enable communication with the cockpit and other ATCOs \\
mole of the systems \\
$\begin{array}{l}\text { ATC systems are considered as being indispensable since they support the } \\
\text { ATCO in controlling air traffic, by providing information and facilitating } \\
\text { communication. They simplify work. }\end{array}$ \\
$\begin{array}{l}\text { ATCOs must rely on systems and sometimes feel they give up } \\
\text { responsibility without 'being in the loop'a. This lack of trust results in the } \\
\text { creation of more safety buffers, which in turn increase the level of } \\
\text { workload. Deskilling may be a consequence of automation. }\end{array}$
\end{tabular}

${ }^{\text {a }}$ being in the loop is an expression often used in ATC and refers to a controller's understanding of the automated system. ATCO = air traffic controller.

was surprising since the radar screen, for example, facilitates information sharing between team members. This perception of the systems seemed to be reasonably consistent among controllers interviewed, implying that they have a similar understanding about the equipment mental model. Some supportive systems are function-specific. For example, the Central Flow Management Unit (CFMU) is only relevant for the supervisor in order to plan the broader traffic flow. Controllers said that they typically know little about the CFMU but yet have a shared understanding about the general usage and functionality of it.

Limits of the system. As any technical system, the ATC system may fail and has limits, for example in the provision of information. Training and experience help controllers to remain vigilant of these limits and sensitive to incorrect data or any equipment malfunction. Controllers who do not fully trust the system will apply backup procedures and often at the expense of a considerably higher workload. On the other hand, controllers who leave all the work to the system may become unable to 'manually' solve a problem in case of system failure (deskilling). The right amount of trust in the system plays therefore a crucial role in the interaction between a controller and the system.

In sum, the knowledge elements of the equipment mental model are consistent with descriptions found in the literature (e.g. Cannon-Bowers et al., 1993). While this mental model type is often disregarded in research as a distinctive mental model type, it appears to be an important separate knowledge type in system-driven environments such as ATC. With other words, the functionality to shared equipment knowledge is high in ATC as the system is the common work environment of all controllers.

The task mental model. The ATC task is strongly determined by formal procedures and legal regulations, which constitute the so-called task mental model. Interviewed controllers stated that accurate and similar knowledge about task procedures, rules, and policies is essential for their work. 
Chapter 2

Table 2.2

The Task Mental Model

Definition: knowledge about task procedures and strategies under varying circumstances

Task procedures Task procedures are formally defined by safety regulations. For example, the military must be contacted if loss of contact with an aircraft lasts for more than two minutes.

Generic guidelines Guidelines are informally described procedures and methods. Examples are procedures for strip management or how to handle situations such as handover, briefings and emergencies.

Likely contingencies/ Likely emergencies can arise through external circumstances (e.g. unusual occurrences thunderstorm), due to ATC (e.g. loss of control), or via third parties (e.g. pilot ignores clearance instructions)

Likely scenarios Likely scenarios include predictable situations (e.g. daily morning peak) and expected events (e.g. bunch traffic) that, compared to likely contingencies, do not constitute a risk.

Task strategies Task strategies aim to increase safety and efficiency and are based on:

- operational requirements (e.g. increase separations between aircraft if approach adjusts approach times)

- mental states (e.g. use your gut feeling, i.e. first thought is often right)

- $\quad$ team composition (e.g. split sectors according to your personnel)

- $\quad$ experience (e.g. fall back on your skills in unknown situations)

Environmental constraints

Environmental constraints occur by changing weather or traffic volume. They lead to uncertainty and increase the interdependencies among team players.

Organisational Organisational culture specifies convention, beliefs and opinions. For influences example, whether male and females are considered as equally competent is influenced by the organisational culture.

Cultural influences Cultural differences based on nationality affect factors such as hierarchies, punctuality and conscientiousness.

Therefore, a task mental model (see Table 2.2) was perceived as an important distinctive type to be included in the SMM framework.

Task procedures. The task mental model contains knowledge about specific procedures of how to accomplish the task successfully and under different circumstances or environmental constraints (see also "likely contingencies" and "likely scenarios" in Table 2.2). An example of a fundamental procedure is: Aircraft separation criteria require a minimum horizontal separation between aircraft of 5 nautical miles and a vertical separation of 1000 feet. This procedure has an immediate effect on traffic capacity, sector planning and tactical task execution, and thus affects decision making at all positions in the ATC team.

Guidelines. Many guidelines have been established to facilitate consistent performance and support solutions in a number of situations. However, the controllers interviewed maintained that it is impossible to define strict procedures to cover all situations or scenarios in the complex task that is faced by an ATC team. Indeed observations confirmed this statement. It was stressed that guidelines 
are only used adequately and successfully when controllers have both correct and shared knowledge about these guidelines. For example, during a shift change, controllers are expected to convey relevant information to their successors. Whether this happens may depend on the time constraints during the shift change, and on a controller's ability to evaluate information as relevant.

Task strategies. Task strategies are another aspect of the task mental model type. Task strategies are not officially defined and regulated. Controllers interviewed reported that they develop personal strategies and base them on the experience they gain during training sessions and operational situations. Some strategies are straightforward and quickly acquired by trainees. An example provided was sequencing of a slow aircraft behind a faster aircraft. However, some strategies only apply to particular situations and require considerable task experience combined with experience to interact with team members. Interviews and observations revealed that knowledge that is less formalised is harder to acquire correctly and is less likely to become shared knowledge among ATCOs.

Organisational and cultural influences. Interviewees stressed that task execution is further influenced by the organisational culture in which the controller operates. For example, a high commitment to safety (safety culture) affects how tasks are accomplished and which strategies are applied. Similarly, a learning-oriented environment and a culture in which mistakes are acknowledged (error-management) and not punished (justice culture) may facilitate high task performance. In addition, knowing the cultural background of fellow team members increases the understanding for each other's behaviour (e.g. the acceptance of hierarchies). This should be openly discusses between team members.

In sum, the knowledge elements related to task execution in ATC agree in general with earlier definitions of the task mental model (Cannon-Bowers et al., 1993). However, the task mental model in ATC stresses strict task procedures as an important element. Failing to comply with procedures or violating or ignoring strict rules is viewed as an extreme safety risk. A second important difference with other team environments concerns task knowledge aspects specifically shaped by organisational and cultural influences. These site-specific knowledge elements are viewed as crucial for task execution and thus need to be added to the ATC specific task mental model.

The team mental model. The interview sessions revealed that accurate and shared knowledge about the skills and attitudes of fellow team members are viewed as considerably important in ATC. A good understanding of each other's competencies enables the team to work together smoothly, even in stressful situations such as during peak times or bad weather conditions.

Controllers' competencies. The team mental model (see Table 2.3) increases the insight that individual controllers have into how their fellow team members function. In general, ATCOs expect that their colleagues are well-trained, and act professionally and according to safety regulations. However, the interviewed controllers acknowledged the existence of individual differences in knowledge, skills and attitudes (KSAs). In fact, team knowledge seems to facilitate that these 
Chapter 2

Table 2.3

The Team Mental Model

\begin{tabular}{ll}
\hline Definition: knowledge about team members' functioning \\
$\begin{array}{l}\text { Controllers' } \\
\text { knowledge, skills \& } \\
\text { attitudes }\end{array}$ & $\begin{array}{l}\text { Controllers expect that every colleague is competent and fulfils the } \\
\text { knowledge requirements. Experience is essential for creative strategies in } \\
\text { complex situations. Attitudes play a big role in how you deal with others } \\
\text { (e.g. Germans adhere to rules) }\end{array}$ \\
Controllers' preferences & $\begin{array}{l}\text { - Personal (e.g. prefer busy shifts and get easily bored during quiet } \\
\text { periods) } \\
\text { - Operational (e.g. prefer direct routing) }\end{array}$ \\
Insight into each other & $\begin{array}{l}\text { Insight into each other (e.g. "planner tries to look into executive } \\
\text { controller's head") diminishes workload via the anticipation of } \\
\text { information needs of team members. This is more difficult for team } \\
\text { members at a separate location or ATC centre. }\end{array}$ \\
Mutual trust & $\begin{array}{l}\text { Trust - 'elbow coordination' - is fundamental for teamwork. It has } \\
\text { positive effects on qualitative and time-efficient information sharing, and } \\
\text { trust creates a safe working atmosphere. A lack in trust results in more } \\
\text { individual and more demanding work. }\end{array}$ \\
\hline
\end{tabular}

differences are recognised, and this understanding develops through mutual work experience and personal relationships. Knowledge about the differences between controllers makes it possible to produce flexible adaptations of how tasks are distributed during a work period, but the perceived differences between ATCOs also often affect the amount of experienced workload.

Controllers' preferences and insight in each other. The interviews showed that in addition to knowledge, skills and attitudes, also knowledge about personal and operational preferences of team members play a role in teamwork in ATC. For example, knowing a colleague's preference for a more pro-active working style implies that this colleague also favours one strategy above another. The controllers interviewed emphasised that knowing each other's preferences is considered crucial team knowledge, as it means that one has insight into the other's thinking and that such knowledge leads to a better mutual understanding. For example, the planner may try to "look into the head" of the radar controller to ease the radar controller's work by taking over some radio communication or plan more accurately in complex situations. This knowledge may pertain to an immediate colleague controller but can also involve aircraft pilots. Recognising a pilot's mind-set during an emergency will prompt a controller to speak calmly and slowly to comfort the pilot and ensure safety.

ATCOs' knowledge of the differences in working attitude between teams of separate control centres also may facilitate ATC. The controllers interviewed mentioned examples that involved the cooperation with adjacent control centres. From the perspective of the controllers, ATCOs of other centres are sometimes less flexible and more committed to standard procedures. This knowledge 
affects how such a controller transfers traffic to another sector. Although differences in attitudes may even cause disagreements, controllers emphasised that safety and professionalism always have the highest priority.

Mutual trust. In some situations it is not possible to have instantaneous knowledge about team members' skills, attitudes and preferences. In such situations, mutual trust is a crucial element of the team mental model. Interviewed controllers described this as "elbow coordination" - as they blindly trust and rely on each other during teamwork. A lack of trust may instead result in individualistic work, more demanding back-up behaviour and consequently a higher workload.

In sum, the team mental model that applies to ATCOs differs in several ways from a generic team mental model type (Cannon-Bowers et al., 1993). The present team model makes a difference between a controller's personal and operational preferences. While operational preferences are mostly more openly shared, personal preferences refer to a rather hidden working style that is only shared after some teamwork experience. The team mental model type also excludes 'team member tendencies' as a specific knowledge element because it was not possible to differentiate this knowledge to knowledge pertaining to team members' preferences. Two knowledge elements, 'insight into each other' and 'mutual trust' appear to be essential in the team model for ATC. These two elements were repeatedly mentioned as such during the interview and were therefore included in the present team mental model type.

The team interaction mental model. The team interaction mental model (see Table 2.4) consists of knowledge about communication patterns and coordination practices. The interaction model refers to a controller's role in a team and the interaction within and between teams. It typically differs from the team mental model, which relates to knowledge on the other team members' competencies. The team interaction model seems to play an important additional role in ATC. The ATCOs interviewed maintained that the knowledge about how to interact with other team members, and with cockpit crews and other ATC centres strongly determines the efficiency of air traffic management system.

Roles and interdependencies. ATCOs interviewed stressed that both the exposure to the task and working in a team are necessary to increase the understanding of team interaction knowledge. They learn how to fulfil and adopt their role effectively, and gain a better understanding of the strict interdependencies with the other roles. For example, the performance of the executive controller partly depends on the quality of the planning of the planner controller.

Information exchange. The team interaction mental model further captures knowledge on how to communicate within the team, and contains details on where information comes from (information sources), via which medium it is communicated (communication channels) and between who information is communicated (information flow). For example, there are specific rules and procedures to use radiotelephony for giving clearances to take off or land an aircraft, and for transfer of control of an aircraft to a colleague or to an adjacent control centre. The controllers interviewed reported that 
Chapter 2

Table 2.4

The Team Interaction Mental Model

Definition: knowledge about coordination and communication among team members

Roles \&

Responsibilities

Pertains to the tasks and responsibilities of all functions. For example, the radar controller services all general air traffic flights. A controller's experience and personal competencies determine how the individual role is fulfilled.

Role interdependency High interdependency exists between ATC roles. For example, the planner controller filters information for the radar controller. "You must do it together".

Information source Information can be extracted from two sources:

- The system (e.g. flight information from radar screen)

- Other team members (e.g. ask experienced colleague for advice)

In general the credo is: "Use all information sources that are available"

Information flow Information flow is often formalised (e.g. announcement of start of morning peak: tower $\rightarrow$ approach $\rightarrow$ area control). The 'how' determines the success and efficiency of information transfer (e.g. speed, intonation, timing)

Communication Most information is transferred verbally (via radio, telephone or personal medium contact) or non-verbally (e.g. indicate something on screen). Textual communication, i.e. datalink, is becoming increasingly prevalent.

Giving and receiving Feedback is provided and received within a team of ATCOs on two feedback levels

- Operational (e.g. debriefing after incident, meteorologist predicts storm, ATC warns pilot, and pilots confirms turbulences)

- Personal (e.g. supervisor report on manning during the hand-over to the other supervisor to give him/her a picture of the level of competencies)

they are heavily trained to abide by these rules and procedures and expect that all controllers nurture a common understanding about these aspects. In fact, communication failure is one of the most frequent causes of incidents in aviation (Stedmon et al., 2007) and controllers believe that the reason for communication failure lies in the inaccurate application of procedures and is rarely due to a lack of knowledge.

Information flow. Figure 2.2 represents a description by the controllers interviewed of how the several roles in ATC are often mutually dependent and how information flows within an area control team. All team members have access to task specific information from the system.

Consequently, they have different overviews of the current traffic situation due to the hierarchical order of the functions they fulfil within the team. For example, the coordination between area control and approach is established by two individuals, namely the supervisor area control centre (SUP ACC) and the supervisor approach (SUP APP) who is responsible for landings. Both these supervisors have more general updated traffic knowledge than the individual controllers. The SUP ACC receives information about the traffic capacity from the flow management position (FMP) and leads the area 


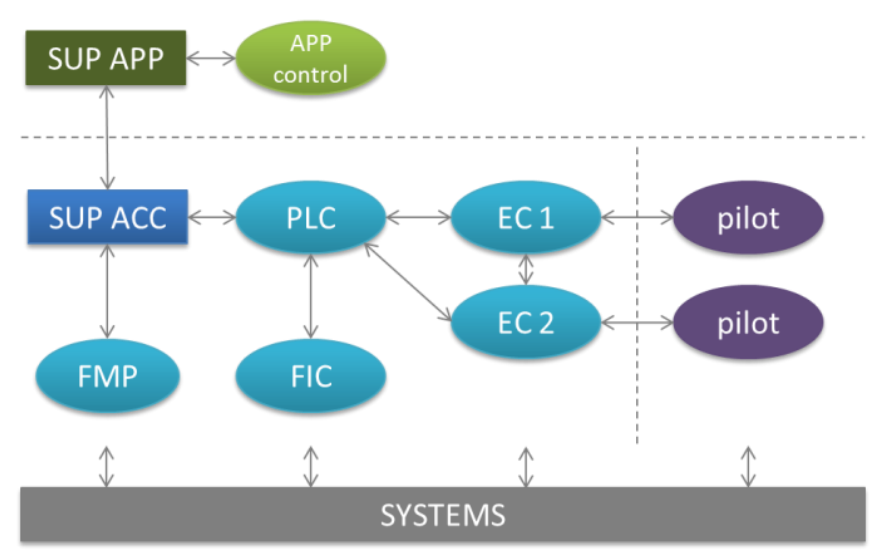

Figure 2.2. Role interdepencies and information flow in an area control team SUP $=$ supervisor; APP $=$ approach $; \mathrm{ACC}=$ area control centre; FMP $=$ flow management position; $\mathrm{PLC}=$ planner controller; FIC $=$ flight information centre; $\mathrm{EC}=$ executive controller.

control (ACC) team consisting of planner (PLC), executive controllers (EC) and flight information centre (FIC) controller. Controllers focus on individual traffic and indicate when their workload starts to increase, as this may become a safety issue. The planner controller filters information and plans the right amount of traffic for the executive controllers, who in turn give clearances to the pilots to transfer to the approach area. Pilots can also request specific routes in which case a controller will check, and confirm and activate, or decline such an option.

Communication style and feedback. The controllers interviewed emphasized that the communication style and even the actual voice and intonation, are important elements in information exchange. For example, the quality of a message may be impeded when a number of fast subsequent or concurrent verbal messages have to be understood. In such a situation, the receiver of a message has to repeat the message (a so-called read-back) to provide feedback to the sender that the message was understood. Providing feedback is part of the team interaction mental model as controllers repeatedly mentioned it as being essential to successful team performance. The controllers stressed that giving and receiving feedback improves mutual understanding of each other's actions and positively affects individual and team behaviour.

In sum, the team interaction mental model type mainly differs from existing typologies due the addition of two knowledge elements, namely information flow and giving and receiving feedback. These elements are particularly important in ATC since the reliability of information is crucial for safety. Due to a formalized information flow and the read-back procedures, controllers can actively ensure that no information becomes lost or is misunderstood.

The situational mental model. The situational mental model (see Table 2.5) emerged from the data collection and appears to be specific to the domain of ATC. This model reflects the specific dynamics in an ATC situation and as such adds to conventional typologies in the literature (CannonBowers et al., 1993). Situational knowledge may constitute a combination of several mental models. It 
Chapter 2

Table 2.5

The Situational Mental Model

\begin{tabular}{ll}
\hline Definition: knowledge about situation assessment and sense of time \\
\hline Situation assessment & $\begin{array}{l}\text { Both earlier (by experience or handover) and current (radar image) } \\
\text { information contribute to an accurate and up-to-date representation of the } \\
\text { airspace. This includes for example, knowing where an aircraft is located. }\end{array}$ \\
$\begin{array}{l}\text { Aircraft related } \\
\text { information }\end{array}$ & $\begin{array}{l}\text { Knowing current aircraft performance (e.g. maximum speed) and with } \\
\text { actual aircraft information (e.g. heading). }\end{array}$ \\
$\begin{array}{l}\text { Information sharing at } \\
\text { right point in time }\end{array}$ & $\begin{array}{l}\text { The timing of information sharing is crucial. For example, information } \\
\text { should be only transferred when receiver has enough available mental } \\
\text { capacity to process the message properly. }\end{array}$ \\
\hline
\end{tabular}

is based on static knowledge that becomes part of an applied strategy as dictated by the situation. A controller interviewed clarified that a sequencing strategy is meant to string together a number of aircraft in a holding pattern and as such is based on unchanging knowledge about these aircraft but is also highly situation specific. For example, a Fokker 100 airplane has a less powerful engine and would usually be second in a holding sequence after an Airbus A320. This strategy changes when an emerging heavy headwind more strongly affects the much bigger A320 than the small Fokker 100. In this situation the Fokker would be positioned in front of the airbus in the sequence.

Situation assessment and aircraft related information. This situation mental model contains highly transient information that assists a controller to keep an updated mental picture of the traffic situation. The knowledge included in the situational mental model enables an ATCO to make adequate decisions as it helps the early recognition and interpretation of cues that indicate situation changes. The situation model needs to be quite specific on knowledge content and on how to assess situations effectively, and therefore extends the earlier notions that were limited to situational awareness (Endsley, 1995). Concrete examples of these knowledge contents are the location, heading and speed of a specific flight at a specific moment in time, the various runways in use, or the current weather situation that may affect a safe approach.

Time-critical information. Another important element of the situation mental model is the understanding of time-dependent information sharing. Timing refers to whether and when information should be exchanged with team members. Timed information sharing is therefore critical and an inadequate timing can have serious consequences for both safety and efficiency. A controller may decide whether information should be shared depending on the receiver's level of workload and processing skills, or on the relevance of the information to be conveyed.

Interdependency with other models. Situational knowledge may constitute a combination of several mental models. It is based on static knowledge that becomes part of an applied strategy as dictated by the situation. An controller interviewed clarified that a sequencing strategy is meant to string together a number of aircraft in a holding pattern and as such is based on unchanging knowledge about these aircraft but is also highly situation specific. 
In sum, the situational mental model combines knowledge of common aircraft performance, current avionics and an update of the situation, which all intervene to lead to adequate and often situation-specific decisions. It thus has a unique relevance to the ATC environment due to its dynamic and time-restricted nature of air traffic. Moreover, it is crucial that team members share this knowledge.

\section{Applying Shared Mental Models in Varying Air Traffic Control Contexts}

This section delineates the findings pertaining to the third research question. It describes the applicability of acquired knowledge structures in changing situations (also non-nominal situations) and different team constellations. Since ATC is highly dynamic, it is important to be complete in covering the variety of situations that ATCOs may encounter when applying their knowledge, because the context can influence shared knowledge structures (Kozlowski, et al., 2009). Particularly, field observations in both normal and non-routine situations contributed to answering the third research questions as they comprised situations that were difficult to verbalize otherwise and made interviewing less appropriate.

Variety and Dynamic Application of Mental Models. A notable challenge of the ATC task is the flexible behaviour required by the dynamic performance demands. The number of possible situations in ATC is quite large and every situation requires flexible and adaptive application of knowledge. The observations showed that the dynamic nature of the ATC task is due to the combination of both quick and relatively slower alterations in airspace. Quick changes may include the rapidly varying constellation of the aircraft that are present in the airspace and includes new aircraft entering and leaving an airspace sector constantly. Slower changes may involve altering sector borders due to temporarily active military areas, or the introduction of new runways at the airport.

In addition, knowledge application and teamwork processes need to be adapted to varying team members as a consequence of flexible teams with altering team composition as is often the case in ATC. Strategies, communication patterns, and work methods must be adapted to both airspace capacity and team members. Observation during crisis management training revealed that different teams showed different behaviours, decisions, communication patterns, or usage of resources despite that the same scenarios were run. However, no team was necessarily more successful than the other, as noted in the debriefings. This illustrates that knowledge can be broadly applied and that behaviour must be adapted to decisions made in particular situations. To fulfil these demands, it is assumed that mental models are constantly updated, adjusted, and aligned with others' mental models in these training situations and eventually will result in accurate and similar SMMs.

This finding emphasised the difficulty to capture team cognition. An analysis of cognition is never complete and it remains a challenge to capture in detail all facets of a complex tasks. In this study, we tried to be as complete as possible by applying a multi-method approach that elicits individual and team knowledge through different methods and in various circumstances. 

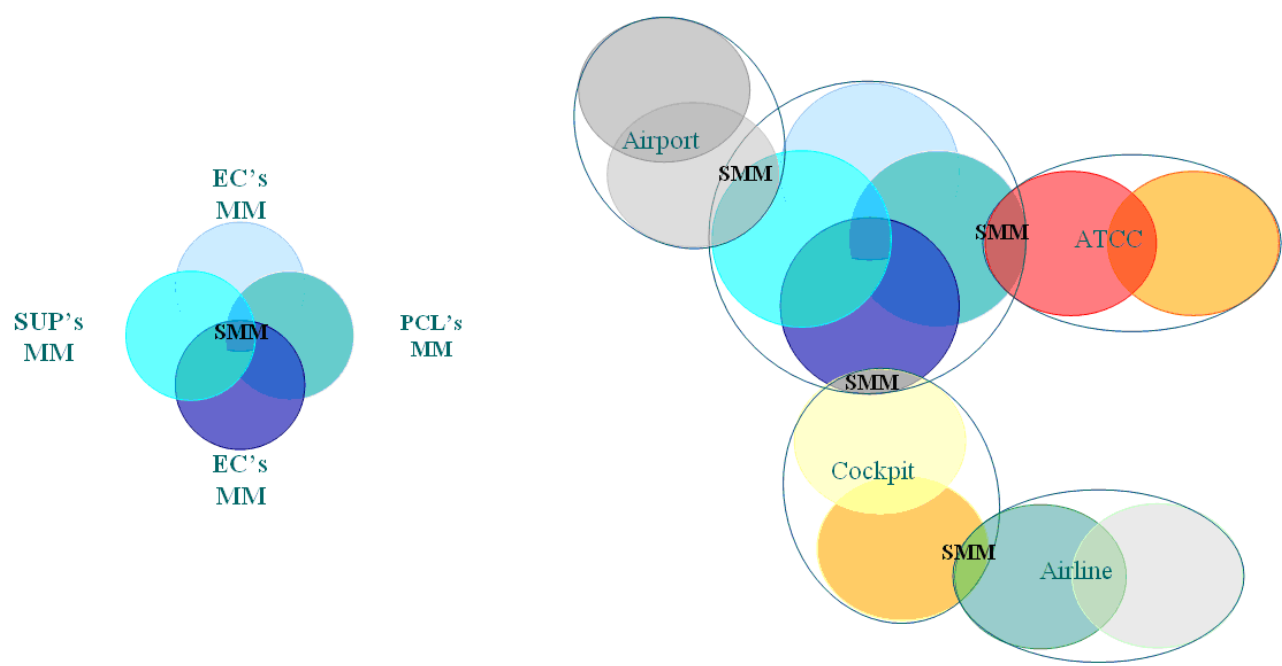

Figure 2.3. Schema of inter- and intra-team relations

$\mathrm{MM}=$ mental model $; \mathrm{SMM}=$ shared mental model; $\mathrm{SUP}=$ supervisor $; \mathrm{EC}=$ executive controller; $\mathrm{PCL}=$ planner controller, $\mathrm{ATCC}=$ air traffic control centre

Mental Models in Non-Routine Situations. The challenge of non-routine situations is that they can rapidly escalate if not handled properly. Often time pressure is high and consequences of an error are severe in these situations. In non-routine situations it is particularly important to have accurate and similar mental models to ensure smooth and efficient teamwork (see also Malakis, et al., 2010b). Controllers observed found it more difficult to understand system's state and predict its behaviour in uncertain situations. It appeared that the simulated situations could be resolved if team members displayed quick reactions, implicit communication and intuitive coordination.

According to the interview data, non-routine events demand high amounts of mental capacity to control traffic safely. Mutual understanding of the task and each other's behaviour is of high importance. Also during crisis training, performance was perceived as successful when the supervisor and the assistant "complemented each other but still fulfilled their own roles and had a distinct task distribution" - as one of the supervisors stated. Moreover, frequently used knowledge not always applied to non-routine situations. Consequently, existing knowledge needed to be adapted to match the requirements of handling a unique situation. This adaptation process further highlights the need for flexibility of mental models.

Intra- and Inter-Team Mental Models. In ATC, sharing similar mental models is not restricted to the individual teams of ATCOs but knowledge must also be shared with other teams within the air traffic management system, such as cockpit crews or ATC personnel at adjacent control centres. Figure 2.3 depicts a schematic overview of inter- and intra-team interdependencies in ATC. The left side of the graph represents a typical team of ATCOs consisting of a supervisor (SUP), two executive controllers (EC) and one planner controller (PCL). The knowledge of an individual team member is represented by a circle and the overlap of circles signifies SMMs within that team. The 
right side of the figure represents additional teams in the air traffic management system, such as a cockpit crew (orange). These teams share knowledge among team members, but also share knowledge with other ATC teams or with an airline team (grey).

The controllers interviewed find it crucial to share an understanding of the goals and tasks distribution when teams are operating at different locations. A controller explained: "The cockpit crew may favour flying efficient and direct routes, but our primary responsibility is to ensure safety and efficiency of the entire system. This may mean that individual clearances not always match the specific preferences of a cockpit crew, but pilots also understand that ATC makes decisions in view of the entire airspace." This implies that insight into each other's responsibilities and goals facilitates mutual understanding of behaviour.

\section{Discussion}

The present study provides an overview of the knowledge structures of ATCOs that capture a distinct number of SMMs. Such an overview of domain-specific knowledge is important for two reasons. First, a detailed understanding of shared knowledge in ATC may help to improve team performance and may lead effective training design in this domain (Malakis et al., 2010b). Second, it contributes to theory development as most SMM typologies are based on theoretical assumptions and await empirical confirmation (Mohammed et al., 2010; Smith-Jentsch et al., 2005). The present results show that SMM theory is adequate as a starting point to study shared knowledge in an operational context. SMM theory describes multiple types of SMM that team members potentially share. However, what knowledge specific teams must actually share remains unclear and requires empirical investigation. SMM theory is therefore limited in its application to an operational context and this study helps to increase this practical applicability.

This study applied a CTA that was based on three qualitative data collection methods, namely a preliminary conceptual modelling, expert interviews and observation sessions of ATC teams in different situations. Based on contemporary notions of the characteristics of SMMs (Cannon-Bowers et al., 1993; Kozlowski et al., 2009), three research questions were addressed to capture knowledge structures, the functionality of knowledge sharing and the usage of knowledge in the dynamic context of ATC. Knowledge structure referred to how knowledge of ATCOs is organised in separate types of mental models (1st research questions). The functionality of knowledge sharing focuses on when it is relevant in ATC to share knowledge (2nd research question). Finally, it was investigated how knowledge is used and shared within the domain-specific dynamic contexts of ATC (3rd research question).

We developed a domain-specific framework of SMMs as based on the incremental knowledge gathering along the three collection methods. The framework differentiates five types of SMMs that appeared to be relevant to teams of ATCOs. These types of SMMs are (1) Equipment Mental Model, (2) Task Mental Model, (3) Team Interaction Mental Model, (4) Team Mental Model, 
and (5) Situational Mental Model. This framework is both similar to and different from earlier proposed typologies (Cannon-Bowers et al., 1993; Smith-Jentsch et al., 2005). Similarities involve the distinction between SMM types, but these similarities remain general and necessarily domain aspecific. The differences readily emerge when the mental model types become more detailed via the domain-specific knowledge analysis in ATC.

The most striking finding concerned knowledge as part of the situational mental model. It pertains to knowledge that is specific to a situation and is thus often highly transient. Situational shared knowledge is rarely considered in the literature as it only fits highly dynamic team environments (e.g. Waller et al., 2004). The situational mental model type proved to be an essential contributor to teamwork in ATC. It helps controllers to provide time-critical information to fellow team members at the right point in time and assess dynamic situations in a similar way. This presumably positively affects ATCOs' levels of workload and their team performance (Salas, Rosen, et al., 2007). Training concepts for ATCOs should include practical exercises to acquire and share situational mental models.

An important basic shared knowledge structure of ATCOs is the equipment mental model. This type is often disregarded in other domains (e.g. Kahneman, 1973; Peterson, Mitchell, Thompson, $\&$ Burr, 2000) and never mentioned in more general descriptions of shared knowledge structures (Mathieu et al., 2010; Smith-Jentsch et al., 2001). ATCOs interviewed emphasized that the systemdriven environment they are working in requires a similar and accurate understanding of the role, usage and limits of the system. Similar as trust among team members, trust in the system is vitally important. Although equipment knowledge is mainly theoretical from nature, hands-on experience with the system may contribute to a better common understanding of the system among controllers.

This investigation also provided some additional ATC-specific insights into the mental model types pertaining to the task, the team and team interaction. The task mental model comprises knowledge about rules and procedures and is particularly important in the safety-sensitive ATC environment. This makes sense as the controllers have to function in a highly dynamic and procedureoriented environment (Langan-Fox et al., 2009; Neal et al., 2011). Ignoring or failing to abide to procedures and rules is a high risk to safety in this environment. It is essential that team members trust their colleagues in regards to their knowledge and the correct application of procedures. Knowledge about rules and procedures should strongly be shared in ATC to ensure safety in air traffic (cf. Malakis et al., 2010a)

On the basis of interview data, we also expanded the task mental model type with knowledge elements pertaining to organisational and cultural influences. The interviewed controllers repeatedly reported that cultural conventions often determine the interpretation of a situation and may therefore also affect how a team executes a designated task. Teams of controller that work in the same organisation, and are subjected to the same culture in which they have to operate, may therefore also have more similar shared knowledge (Cannon \& Edmondson, 2001). Future research should look 
more closely into this issue as shared cultural knowledge and conventions are probably not specific to ATC and should be relevant to understand teamwork in other environments.

Also the team mental model in ATC differs in a number of ways from existing knowledge typologies (Cannon-Bowers et al., 1993; Smith-Jentsch et al., 2005). Earlier typologies stress that team member preferences are part of shared team knowledge (Cannon-Bowers et al., 1993). The present study expands this notion by differentiating between personal preferences and operational preferences. Personal preferences merely affect the individual working style, whereas a controller's operational preferences directly affect the operation and the effectiveness of the entire team. In effect, knowing about the preferences of fellow controllers makes it possible to anticipate on their choices and behaviours (Salas, Rosen, et al., 2007). As the development of this knowledge is a matter of experience with other team members, ATCOs should frequently train in a team context.

Two additional knowledge elements were found to be important in the team mental model of ATC, namely insight in each other and mutual trust. These elements appear to be specific to ATC. They help team members to work together in a demanding and in a time-sensitive environment, but without the explicit need for communication. These knowledge elements are typically absent in earlier suggested SMM typologies (Cannon-Bowers et al., 1993) and future research should verify if these knowledge elements are applicable in other team environments.

Finally, the earlier reported team interaction mental model type was added by two knowledge elements, namely information flow and providing/receiving of feedback. The flow of information is formalised and often strictly regulated in ATC to ensure both rapid and transparent communication (Malakis et al., 2010a). Interviewed controllers confirmed that accurate and shared knowledge about the information flow is essential for smooth teamwork. In addition, giving and receiving of feedback is indeed an emphasized element in ATC, and has not been part of earlier descriptions of SMM types (e.g. Mathieu et al., 2010). The emphasis on feedback increases the understanding of controllers of how their actions affect their team members (Salas, Rosen, et al., 2007). This shared understanding is crucial due to the high interdependency within ATC teams.

The above additions and refinements of earlier shared knowledge typologies refer to structural and functional knowledge aspects. The current analyses also show that shared knowledge structures may be shaped by situational influences within the ATC context. Interviews and observation revealed three main findings. First, shared knowledge in ATC is often not static. Interviewed controllers emphasized that a situation rarely remains the same. Therefore is an understanding of the current situation only possible when knowledge is regularly updated, and is adjusted, aligned and shared with fellow team members (Uitdewilligen et al., 2013).

Second, non-routine situations are often challenging as they pose a high demand on updating of (shared) knowledge, which is necessary because learned strategies and conventions mostly do not apply in these circumstances (see also Sander et al., 2015). Such a situation makes it more difficult to predict team member intentions and behaviour. At the same time, a shared understanding is extremely 
Chapter 2

important in these non-nominal situations, as this makes the situation less demanding (Waller et al., 2004) and makes it possible to spend more mental resources on role-specific tasks (cf. Malakis et al., 2010b).

A third finding that refers to context-specific shared knowledge in ATC, is that shared knowledge structures differ throughout the entire ATM system. These differences make functional sense, and different teams only share the necessary amount of information to ensure effective teamwork and a safe airspace.

\section{Practical Implications}

The present study has a number of important practical implications. The findings can contribute to efficient teamwork in ATC and confirm the applied value of studying SMMs in real team environments. The suggested framework can be useful to evaluate existing training programs on completeness and developing new ideas to further improve teamwork between ATCOs.

The customised mental model types defined in this study may help to define training content and set up lessons with illustrative and meaningful examples specific to ATC. Indeed, the results typically emphasize the need for various task contexts and situations in training, to make it possible to train the flexible application of shared knowledge. A recent example of such an approach is a computer-based training that provide concrete examples of teamwork to improve mental models of submarine attack teams (Smith-Jentsch et al., 2001).

The suggested framework may also be used to diagnose performance issues and explain performance differences between teams in ATC. The framework can show potential shortcomings of team members who fail to anticipate information needs. Additional training may remedy such an issue as these ineffective team members may have insufficient insight into the roles of fellow team members or may have a deviating perception of task goals.

\section{Potential Benefits and Limitations of the Present Approach}

The mixed method approach applied in the present research was appropriate as a basis for a CTA. Preliminary conceptual modelling, interviews and observations were the basis to answer three research questions on the use of shared knowledge in ATC in terms of structure, function and context. This approach started with a conceptual model of the competencies of individual ATCOs in light of the main goals, and work from there to appraise both verbal reports and observed behaviour. Interviews made it possible to infer knowledge structures from the report by experts in the field and observations gave additional insights of how ATC should work in unforeseen situations.

Even though CTA is considered as an effective approach to study SMMs in complex cognitive settings (Mohammed et al., 2010), the qualitative methods of data collection on which the task analysis it based are thought to have certain limitations such as subjectivity. In the present study 
we tried to minimize these limitations through using three different data collection methods. Also, it is important to realize that participants were experienced experts in the field.

The reader should note that only area control ATCOs participated in both interviews and observations. While basic competencies and knowledge are comparable to other functions in ATC, a more complete picture may be gained to include for example approach control in future studies. As a comparison, most other research on cognitive aspects in ATC, either focus on en-route control or have not specified the target group at all (e.g. EATMP, 1999; Niessen \& Eyferth, 2001; Seamster et al., 1993).

The resulting framework identifies (shared) mental models of ATCOs, but this general outline is unable to specify the vast amount of knowledge that is actually shared among controllers. The present outcomes were neither able to specify how knowledge accuracy and similarity relates to team performance in ATC. These relationships should be part of subsequent studies.

To conclude, this study developed a typology that captures domain-specific mental models of ATCOs. This gives insights into their behaviours, knowledge and team processes and can be applied to evaluate team performance and play a part in the development of team training. The results showed the usefulness of mixed methods as a basis for a CTA, and provide empirical support that a number of separate knowledge representations (mental models) provide an adequate description for the general knowledge base of ATCOs. Results implied high flexibility of mental models when they require frequent knowledge updates in a dynamic task environment. Subsequent research should focus on the accuracy and similarity of the mental models among team members and how they affect team performance.

\section{Acknowledgements}

This work was only made possible through the efforts of the contacts at The Dutch Air Traffic Control and the cooperation of the air traffic controllers. We would like to thank all individuals who have been involved in this study. 


\section{Appendix II.I}

\section{Semi-structured interview scheme}

1. Describe a usual working day

What kind of tasks do you carry out?

What activities / sub-tasks are required to carry out these tasks?

How is a task initiated?

What goals are related to these tasks?

What are the most important competencies to carry out your work?

Which tools do you use?

2. Describe a non-nominal or a very complex working day / situation

What was exactly different on this day?

Which competencies were particularly required?

... relate these answers to the previous statements about a usual working day...

3. What makes your work complex?

Which kind of error(s) can occur (due to the complexity)?

What are the possible consequences?

How can you correct mistakes?

4. Teamwork

How would you describe good teamwork?

Who is part of your team?

What are team competencies?

What role do attitudes play in teamwork? Which attitudes are important?

Are you familiar with the KSAs of your team members?

5. Organisation of knowledge

Which knowledge is required based on the situation described?

Which knowledge do others have, that you do not have?

Is it more important that knowledge is distributed or shared among team members?

Why?

6. Information management

What kind of information do you need to carry out your task?

Who is involved in your work?

HMI (role of HMI as a medium)?

How do you communicate with others? Via which channels?

How do you decide when to provide information to others?

Note. KSAs = Knowledge, skills, attitudes; HMI = Human-machine interface 


\section{Chapter 3}

\section{A card sorting study to investigate shared mental models in air traffic control teams as a function of organizational context and work experience}

The aim of this study was to validate the framework of shared mental models developed in Chapter 2 and investigate existing typologies of shared mental models in teams of air traffic controllers. We investigated whether the similarity of air traffic controllers' mental models is affected by the organizational context and whether this relationship is mediated by the level of work experience. One hundred air traffic controllers participated in a card sorting study. Results showed that air traffic controllers share three types of mental models pertaining to equipment, task and team. Similarity of mental models of task and equipment differed as a function of organizational context but only in air traffic controllers with less than 10 years of work experience. 



\section{Introduction}

Working together in teams is essential in many contemporary work environments. These environments are often too demanding and complex to be successfully handled by individuals (Salas, Rosen, et al., 2007). Individuals therefore need both idiosyncratic knowledge of their specific task and shared knowledge with their fellow team members (Cooke, Gorman, \& Winner, 2007). This latter shared knowledge is often viewed as converged individual mental models and is referred to as shared mental models (SMM; McComb, 2007).

SMMs are organized knowledge structures that help team members to coordinate their behaviours and understand their task in a similar way (Cannon-Bowers et al., 1993; DeChurch \& Mesmer-Magnus, 2010a). The relevance of SMMs depends on the tasks and functions a team has to perform (Cannon-Bowers et al., 1993) but it is yet unclear which types of SMMs are relevant for which kind of team. Several typologies of SMMs have been suggested (e.g. Cannon-Bowers et al., 1993; Uitdewilligen et al., 2010), but few researchers have tested the accuracy and utility of these typologies for specific teams in the field. In Chapter 2, we also suggested a typology (framework) of SMMs, which awaits further validation. Another shortcoming in SMM research is that little is known about the factors that cause the development of the various SMM types among team members (Mohammed et al., 2010). As SMMs enhance team effectiveness (DeChurch \& Mesmer-Magnus, 2010b), it is important to learn more about both the types of SMM in real teams and the determinants of these SMM types.

This study has two objectives. The first objective to improve the understanding of the largely theoretically described SMM concept due to a domain-specific investigation of the types of SMMs in teams of air traffic controllers (ATCOs). We focus specifically on ATCO teams because team knowledge is essential for their tasks (Malakis et al., 2010b). In addition, evidence suggests that team effectiveness of complex teams such as ATCO teams, combat teams, surgery teams and teams working in power plants benefits from SMMs (Gross \& Kluge, 2014; Mathieu et al., 2010). The second objective is to advance SMM theory through testing two factors related to SMMs in real teams, namely the organization context and work experience. Specifically, we study the effect of organizational context on team members' SMM and whether this effect is mediated by team members' work experience. Studying these determinants in the field is important because, compared to teams studied in laboratory settings, real teams are genuinely affiliated to an organization and have thorough experience with their task.

\section{Types of Shared Mental Models}

Even though there is a general agreement that SMMs are essential for team success (DeChurch \& Mesmer-Magnus, 2010a), the types of mental models that specific teams share and that thus predict team effectiveness are unclear (Mohammed et al., 2010). Typically researchers suggest multiple 


\section{Chapter 3}

mental model types in teams (Cannon-Bowers et al., 1993; Smith-Jentsch et al., 2005) but these types have only been theoretically derived and have not been simultaneously tested in an empirical study in the field (Langan-Fox et al., 2004; Lim \& Klein, 2006; Smith-Jentsch et al., 2005). A test of the concepts in a field study helps both to discern the usability of the concept and to establish its practical validity. In addition, such a test helps to clarify whether team members' SMM can best be described by multiple types of SMMs as suggested by SMM theory (Cannon-Bowers et al., 1993; Mohammed, Klimoski, \& Rentsch, 2000).

The present study builds on the theoretical framework of domain-specific SMMs for ATCO teams described in Chapter 2 of this dissertation. This framework adds to general notions from the literature on SMMs (Cannon-Bowers et al., 1993; Smith-Jentsch et al., 2005; Waller et al., 2004) and simultaneously leads to domain-specific details. The framework comprises of five mental model types and each type consists of a number of knowledge elements that specify the content of these types. The types distinguish between knowledge on the role and limitation of the equipment (equipment model) and knowledge about task procedures and task strategies (task model). Additionally, two of the five mental model types pertain to aspects of the team, and include knowledge about the competencies of other team members (team model) and shared knowledge of coordination and communication processes (team interaction model). Finally, one type comprises knowledge of the situation and the timing of information sharing (situational model).

Although we developed the framework in cooperation with ATCOs, we have not tested it empirically and we based our findings on qualitative data collections and analyses. This qualitative approach involved that we interpreted knowledge elements provided by expert ATCOs and subsequently allocated these elements to mental model types. Consequently, results based upon our interpretation and the question remains whether subject matter experts also recognise these mental model types as relevant and functional to their work. This is important, as training programs designed to train experts in effective SMMs must be meaningful to these experts to improve teamwork efficiently (Langan-Fox et al., 2004; Mohammed et al., 2010; Smith-Jentsch, 2009).

We may expect that ATCOs' mental models are more condensed as the SMM framework suggests because expert knowledge is diversified and often organized in meaningful chunks (Marsh \& Ahn, 2012). Specifically, controllers may distinguish two types of knowledge, namely knowledge related to the task and knowledge related to the team (Ilgen, 1999). These two types of knowledge

typically describe work in cooperative environments. It is doubtful that controllers separate knowledge concerning the team from knowledge concerning team interaction as this may be too detailed and unnecessary for a practitioner (Mathieu et al., 2000). This also applies to the situational model as its knowledge elements strongly interweave with the executed tasks and team activities. For example, an unexpected change in the traffic situation can require system input, new task strategies and informing other team members (Malakis et al., 2010a, 2010b). ATCOs may thus disperse aspects of the situational model type across equipment, task and team knowledge. In contrast, we expect the mental 
model regarding equipment to be clearly recognisable by ATCOs, as operating and trusting automated systems play a crucial role in their work (Langan-Fox et al., 2009).

In sum, we expect that if we ask ATCOs to allocate knowledge elements of the SMM framework described they will distinguish three types of mental model types pertaining to equipment, task and team. Accordingly, we hypothesize:

Hypothesis 1. ATCOs will distinguish knowledge elements relating to aspects of equipment, task and team, and thus distinguish three different types of mental models.

\section{Organizational Context and Work Experience as Determinants of Shared Mental Models}

While the first hypothesis investigates the types of SMMs that ATCOs use, it provides no insight on how compatible these mental models types are among controllers. Two factors that possibly determine the compatibility of mental models are the organizational context at which the controllers work and their work experience (Kraiger \& Wenzel, 1997). We study these factors in the field where team members have genuine experience with the task and feel affiliated to an organization. To investigate compatibility we draw on mental model similarity as an indicator of SMMs (Marks et al., 2000).

\section{Organizational context}

The present study distinguishes controllers working at two European air navigation service providers. Their knowledge should be generally comparable as they are subjected to the same basic selection and education procedures (Marsh \& Ahn, 2012). All controllers perform relatively equivalent tasks and conduct their work according to European standards (ICAO, 1996, 2001). The ongoing harmonization of the European airspace further increases this need for conformity of knowledge among controllers of separate organizations as they need to cooperate closely (Langan-Fox et al., 2009; SESAR, 2012).

However, differences in organizational context can also affect the way in which team members work together (Arrow, McGrath, \& Berdahl, 2000; Hackman, 2003; Kozlowski, Gully, Nason, \& Smith, 1999) and represent and share knowledge (Uitdewilligen et al., 2010). Each organizational context contains specific rules that reflects the culture of the organisation. Furthermore, each organization may be differently supportive in the development of team members' SMMs (Druskat \& Pescosolido, 2002; Yang, Kang, \& Mason, 2008). For example, team members who work in an organization that encourages teamwork and interpersonal relationships may often have developed effective SMMs. The restriction of opportunities of interpersonal exchange and a focus on individual success may results in less similar mental models between team members (Druskat \& Pescosolido, 2002).

This study tests whether the similarity of ATCOs' mental models relates to the organizational context. We assume that the organizational context influences the similarity of ATCOs' mental models 


\section{Chapter 3}

(Uitdewilligen et al., 2010). This relationship has not been investigated and contributes to the understanding of SMMs and teamwork in different organizations. We test the following hypothesis:

Hypothesis 2. The organizational context affects the similarity of ATCOs' mental models.

\section{Work experience}

The effect of the organizational context on team members' SMMs may particularly concern unexperienced team members. Unexperienced team members still need to develop SMMs and during this developmental phase they will be more sensitive to aspects of the organization such as training guidelines (Burke et al., 2006; Druskat \& Pescosolido, 2002). Experienced team members, on the other side, often have developed rich and accurate mental models that are similar to other team members (Levesque et al., 2001). They have consolidated their knowledge and may therefore be less prone to external influences.

Work experience leads to more knowledge of the task and more shared knowledge with other team members due to the exposure of several task situations and interactions with various team members (Cooke, Gorman, Duran, et al., 2007; Rentsch \& Klimoski, 2001; Smith-Jentsch et al., 2001). Specifically the complex and dynamic nature of the air traffic control (ATC) task involves that ATCOs frequently encounter new situations (Malakis et al., 2010a) and need to adapt to new team members (Malakis et al., 2010b). Only experience may lead to common knowledge of the variety of these situations. Smith-Jentsch et al. (2001) confirmed this relationship in experienced Navy personnel who shared more teamwork mental models than their colleagues who had served a shorter time. Also, the novice-expert literature corroborates this relationship as knowledge structures of team members become both more accurate, complex and diverse over time (Borko \& Livingston, 1989).

We argue that work experience mediates the relationship between the organizational context and SMMs. The SMMs of experienced ATCOs result from experience with the task, a variety of situations and teams, and are less prone to influences of the organizational context. The organizational context may therefore affect SMMs of ATCOs with low work experience but not the SMMs of ATCOs with high experience. Accordingly, we hypothesize:

Hypothesis 3. Work experience mediates the relationship between organizational context and mental model similarity so that differences in mental model similarity of ATCOs of separate organizations diminish with work experience.

\section{Method}

\section{Participants}

In total 100 ATCOs from two European air navigation service providers participated in a card sorting study. Thirty-seven (30 males), worked at organization 1, and 63 (50 males) were controllers at organization 2 . The average age was 36 years $(S D=8)$ and ranged from 23 years to 53 years. 
Controllers at organization 1 provide approach and area control and are responsible for air traffic in the vicinity of the airport. Approach controllers coordinate aircraft leaving or approaching the airport to or from designated airways. After the aircraft have left the airport and have climbed to a certain altitude, area control takes the guidance of the aircraft and is responsible for the separation of aircraft on the airways and the traffic crossing the area above an airport. Similar area control guides aircraft on airways to an airport until they transfer the aircraft to approach control at a certain altitude. Controllers working at organization 2 provide en-route control. They provide cross-border air navigation services in the upper airspace. At all positions at least one planner and one radar controller work together. The planner coordinates with adjacent sectors and is in charge for the planning of an adequate amount of traffic to avoid overload. The radar controller coordinates with all aircraft in the area of responsibility. The main goal at all positions is to ensure safe and efficient air traffic.

\section{Card sorting task}

In order to test our hypotheses ATCOs completed a closed card sorting task (Spencer, 2009) and were instructed to allocate predefined knowledge elements (presented on cards) to predefined knowledge categories. Elements and categories were based on the framework for ATC developed in Chapter 2. Each card represented one knowledge element (e.g. mutual trust, task strategies and cultural influence) and each category captured on of the five described mental model types (e.g. task mental model or situational model). In total, 30 cards and 5 categories were used. A sixth category ('undefined') was made available to allocate cards with no match to any of the SMM categories.

Five subject matter experts checked both card labelling and task usability. This preliminary test led to a further refinement. On instigation of the experts, examples illustrated a number of knowledge elements for a uniform understanding. Additionally, the terminology of some knowledge elements was customized to the terminology used at each of the two organizations. For example, the term New Flight Data Processing System was also represented as Flight Data Processing.

ATCOs performed the card sorting task via a web-based application. ATCOs of each organizational ran a different version of the application, which matched the terminology of their organization. All participants were instructed to perform the task from the perspective of a radar controller to ensure an equal mind-set.

\section{Measures}

Types of ATCOs' SMMs. To reveal whether ATCOs' distinguish among mental models pertaining to equipment, task and team card sorting data were subjected to multidimensional scaling (MDS). MDS creates a spatial representation and expresses dissimilarities between knowledge elements via distances in Euclidean space (Seamster et al., 1997). Knowledge elements per participant were represented in a $30 \times 30$ matrix. Both columns and rows of each matrix represented all knowledge elements and a cell represented a combination of two knowledge elements. We counted the 


\section{Chapter 3}

number of knowledge element pairs (cells in the matrix) that were present in every predefined knowledge type. This yielded the total frequency of all knowledge element pairs in each predefined type per participant.

All knowledge element pair frequencies were summed across participants and each sum was subtracted from 100 (number of participants), which yielded a distance matrix. This means that pairs that never combined in a knowledge type were separated by the largest distance (100). The data were then subjected to the alternating least squares approach to scaling (ALSCAL) method of MDS (Takane, Young, \& de Leeuw, 1976) to find the best fitting representation of knowledge, with knowledge elements forming clusters if they had equal distances from other knowledge elements. A cluster represents a SMM type.

\section{Mental model similarity as function of organizational context and work experience.}

Similarity among team members' mental models was determined by the exact Fleiss' Kappa ( $\kappa)$, also known as the inter-rater reliability among multiple raters (Conger, 1980; Fleiss, 1971). Contrary to other agreement indices, such as inter-rater agreement (rwg,) or phi correlation that use binary values, Fleiss' Kappa is a meaningful statistic to assess agreement via the frequency numbers of knowledge categories.

Differences in mental model similarity of ATCOs of different organizations (hypothesis 2) and with different levels of experience (hypothesis 3 ) were determined through a permutation test (Good, 2000; W.-C. Lee \& Rodgers, 1998). To test organization differences in SMMs, the permutation procedure involved that the card sorting data of the 100 participants was randomly sampled in two groups but under the constraint that 37 participants belonged to organization 1, and 63 to organization 2. The exact Fleiss' Kappa was then determined for each group and the Fleiss' Kappa difference functioned as the test statistic. This resampling procedure was repeated a thousand times to generate a sampling distribution of difference scores, also referred to as the permutation distribution. This sampling distribution assumes that the two groups do not differ in terms of inter-rater reliability (or similarity of mental models).

The next step involved locating the position of the actual Fleiss' Kappa group difference within the generated sampling distribution. In particular, for a one-sided test to be significant at $\alpha=$ 0.05, the value of the observed Fleiss' Kappa group difference should be equal or larger than the different score on the 950th position. We performed separate permutation tests for each of the predefined knowledge categories (SMM type).

We applied the same permutation testing method to assess whether work experience mediates the relationship between the organizational context and the similarity of ATCOs mental models. For this purpose, we divided the samples into controllers with low experience ( $\leq 10$ years) and those with high experience (>10 years) at the two organizations. We chose this criterion based on the 10-year rule of expertise stating that at least 10 years of experience are required to become an expert (Simon \& Chase, 1973). Accordingly, 15 controllers at organization 1 were in the low experience group and the 


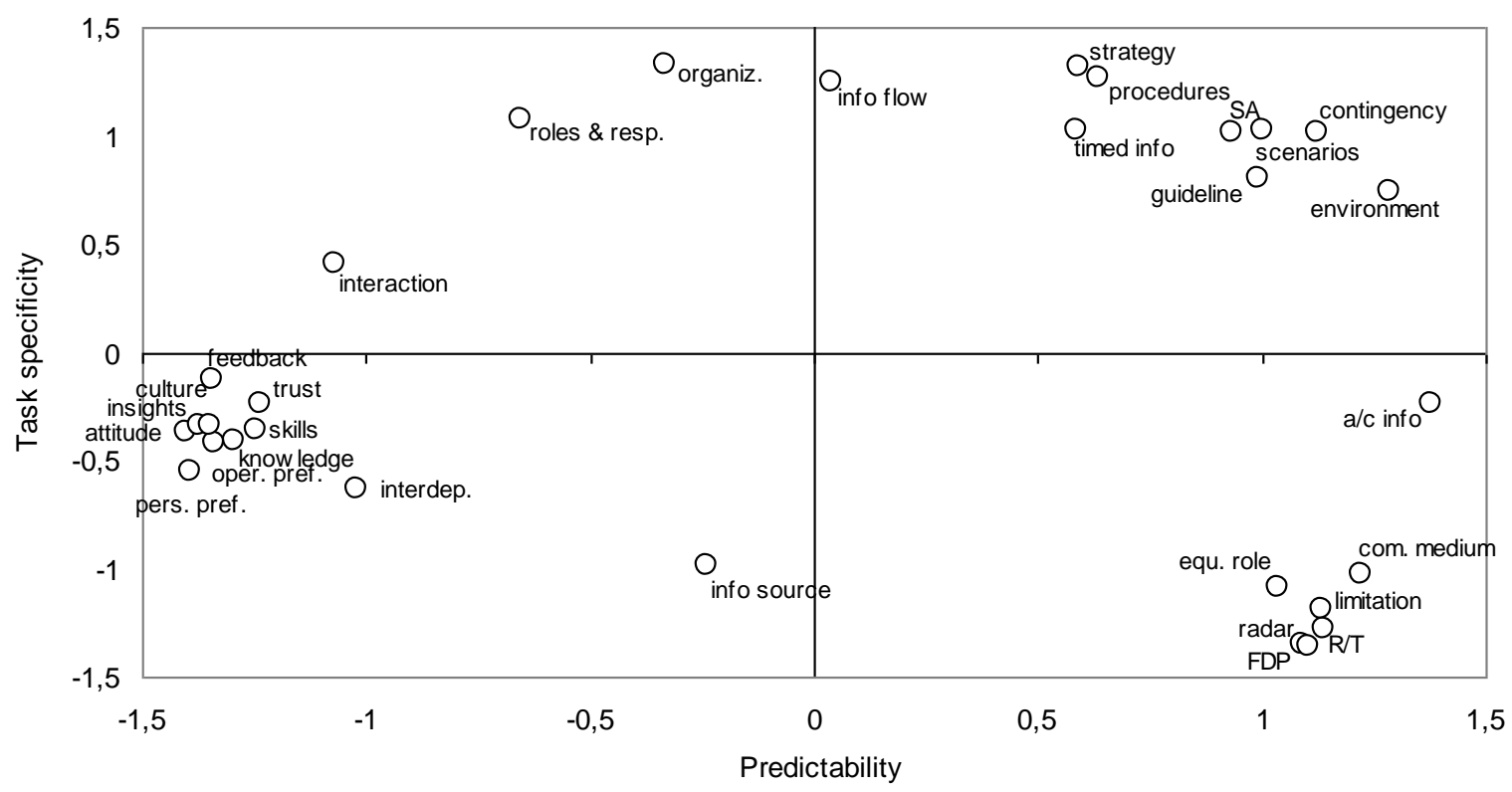

Figure 3.1. Two-dimensional configuration plot of the card sorting task (Euclidian distance model). pers. pref. $=$ personal preferences; oper. pref. $=$ operational preferences; interdep. $=$ interdependency; roles \& resp. $=$ roles \& responsibilities; organiz. infl. = organizational influences; info source $=$ information source; info flow $=$ information flow; $\mathrm{a} / \mathrm{c}$ info $=$ aircraft information; equ. role $=$ equipment's role; com. medium $=$ communication medium; FDP = flight data processing; $\mathrm{R} / \mathrm{T}$ = radio telephony

remaining 22 were in the high experience group. The distribution at organization 2 was 39 controllers in the low experience group and 24 controllers in the high experience group.

\section{Results}

\section{Types of air traffic controllers' shared mental models}

The ALSCAL method of MDS revealed that the data was most adequately presented by a twodimensional solution fit. The best-fitting relationship was estimated based on the dissimilarity of cases $\left(R^{2}\right)$ and the Euclidean distances between them in a q-dimensional space $(d)$. Kruskal's stress 1 formula yielded a stress value of .29 while $R^{2}$ amounted to .72 .

Figure 3.1 shows the two-dimensional MDS solution by depicting dissimilarities between knowledge elements. Knowledge elements that are closer together were assigned more often to the same SMM type by the participants. The configuration comprises three distinctive point clouds in the lower right, the upper right and the left middle. This implies that the controllers categorized domainspecific shared knowledge in three rather than in five distinct knowledge types as supposed by hypothesis 1 . Specifically, knowledge elements in the lower right relate to equipment, those in the upper right relate to task and situation, and knowledge elements in the left middle relate to team and team interaction. Thus, knowledge of the team and team interaction merged into a single representation (team-related). ATCOs also clustered both task and situational knowledge elements into a general task-related SMM type. Four knowledge elements (roles \& responsibilities, organizational 
influence, information flow, information source) remained isolated and located between the main clusters.

Closer inspection of Figure 3.1 reveals a number of further details of how ATCOs represent and share knowledge. The horizontal axis illustrates the distinction of knowledge in two domains: team knowledge and task/equipment knowledge. The left side presents knowledge about team-related aspects, while the right side displays knowledge elements that are part of the factual knowledge of controller. This refers to the actual procedures, task strategies and function of the automated system/equipment. Knowledge that involves either the team or the system, such as information flow and information source, remain in the centre of the horizontal dimension. For example, both a team member and the radar screen can serve as information source about a traffic situation.

The vertical axis ranges from static knowledge, such as about the equipment that always works the same, to dynamic knowledge regarding for example task strategies and situation assessment that may differ per situation. The team-related knowledge elements, such as role interdependency and insight in each other, are in the middle of this dimension. It seems that ATCOs perceive this knowledge as moderately dynamic.

As the MDS revealed that three types best represent ATCOs' SMMs these types were included in subsequent testing. The following analyses thus tested whether ATCOs of separate organizations (hypothesis 2) and with different levels of work experience (hypothesis 3) differed in the similarity of their equipment, task, and team-related mental models.

\section{Organizational context as determinant of shared mental models}

In support of Hypothesis 2, the permutation test revealed a significant difference in mental model similarity between controllers at organization $1(\kappa=.60)$ and organization $2(\kappa=.52 ; p<.05)$. A more detailed analysis for each of the three SMM types showed that similarity of knowledge on equipment was .11 larger for ATCOs at organization 1 than at organization 2 (organization $1: \kappa=.74$; organization $2: \kappa=.63 ; p<.01)$. For task-related knowledge, the difference between ATCOs of organization 1 and organization 2 was .1 (organization 1: $\kappa=.55$; organization $2: \kappa=.45, p<.01$ ). ATCOs of the two organizations did not significantly differ regarding similarity of team-related knowledge (organization $1: \kappa=.62$; and organization $2: \kappa=.60 ; p>.30$ ).

\section{Work experience as mediator of organizational context and shared mental models}

The permutation test to assess hypothesis 3 showed that the difference of general shared knowledge between low experience controllers at organization 1 and low experience controllers at organization 2 was .13 (organization 1: $\kappa=.63$; organization $2: .50, p<.05$ ). This finding was confirmed for equipment knowledge $(\kappa=.79, .62$, Diff $=.1, p<.05)$ and task-related knowledge $(\kappa=.58, .44$, Diff $=$ $.14, p<.05)$, but not for the team-related model type $(\kappa=.61, .60$, Diff $=.01, p>.3)$. Similarity of mental models of high experienced controllers did not differ between organizations. 


\section{Discussion}

The aim of this study was to validate the SMM framework developed in Chapter 2 and investigate existing typologies of SMMs (Cannon-Bowers et al., 1993) in teams of ATCOs. These typologies of SMMs has been suggested as important in teamwork, and particularly in teams of ATCOs, but has not been validated by empirical research. We also considered the similarity of the mental model types and examined whether these are affected by the organizational context at which ATCOs work and whether this relationship is mediated by ATCOs' level of work experience.

The investigation of the different SMM types of ATCOs was based on the SMM framework developed in Chapter 2. This framework includes five types of SMMs, namely equipment, task, team, team interaction and situation. While this detailed distinction is theoretically comprehensible, we hypothesized that ATCOs will distinguish only a restricted number of these types. In line with this expectation, ATCOs differentiated knowledge pertaining to the equipment, the task and the team. This result implies a number of new findings that will contribute to the literature on SMMs.

First, this study shows that the SMM regarding equipment is essential for ATCOs as they distinguish this knowledge from other knowledge types. This type has already been suggested in Chapter 2 and by other researchers (Cannon-Bowers et al., 1993), but awaited empirical confirmation. ATCOs teams depend heavily on the equipment and it makes sense that they identify these aspects of their work as separate from other knowledge. It is likely that other teams working in system-driven environments, for example military teams, also have distinctive representation of equipment knowledge. Despite this relevance of equipment SMMs, previous studies have not investigated this type of SMM specifically but typically as part of the task SMM (Lim \& Klein, 2006; Mathieu et al., 2010). The present results imply that this might not be sufficient to understand the knowledge structures of teams working in complex, system-driven environments.

The second implication of this study is that ATCOs knowledge about other team members and team interaction is represented in a single team-related SMM type. A further distinction into team SMM and team interaction SMM seems to be too detailed and unnecessary for the subject matter experts involved in this study. This finding is in line with the literature that experts knowledge is organized in meaningful chunks and not necessarily very detailed in nature (Marsh \& Ahn, 2012). The finding also corroborates several studies on SMMs, which often assess team members' SMMs of team and of team interaction as one merged team-related SMM type (e.g. Banks \& Millward, 2007; Lim \& Klein, 2006; Mathieu et al., 2010).

Thirdly, the present results show that ATCOs clustered knowledge elements regarding task and situational elements into a general task related category, suggesting a general task SMM. This outcome is in line with our expectations that controllers do not recognize situational knowledge as a separate functional SMM type but disperse these knowledge elements across alternate SMM types. Indeed, situational knowledge especially seems to be closely connected to the executed tasks and team activities. This interlaced nature makes it difficult to operationalize situational knowledge in a distinct 


\section{Chapter 3}

SMM type and explains why few studies actually tried to assess situational SMMs (e.g. Cooke et al., 2001; Rico et al., 2008; Waller et al., 2004). As situational knowledge remains important in dynamic, quickly changing environments, such as ATC, we should not disregard this type of SMM but study, test and train it as part of task and team knowledge.

A last implication of the present analysis of the SMMs of ATCOs' within the framework is that these can be described along two dimensions. One dimension specifies team-related versus equipment/task aspects and the second specifies static versus dynamic knowledge elements. The first dimension supports early and current ideas on SMMs, which suggest that SMMs can be described in terms of task-related aspects including the equipment/technology and team-related aspects (Klimoski \& Mohammed, 1994; Mathieu et al., 2000). The second dimension that became apparent in the present analysis confirms that SMM types are not equal in their stability. Due to for example new team members, varying team compositions and changing skill levels of team members the team-related mental model type is more dynamic. Knowledge regarding for example procedures and system use changes less frequently so that task and equipment mental model types are more static in nature (Cannon-Bowers et al., 1993; Mathieu et al., 2000; Rentsch \& Hall, 1994). Our study for the first time presents empirical support for this idea.

The similarity of ATCOs' mental models depended on the organizational context of controllers. ATCOs working at approach/area control had more similar mental models than controllers working at en-route control. This specifically applied to equipment and task knowledge. This finding confirms the idea that the organizational context can shape knowledge structures of individuals and can subsequently affect the development of their SMMs (Druskat \& Pescosolido, 2002; Langan-Fox et al., 2004; Uitdewilligen et al., 2010). For example, organizational support for heedful interrelations and opportunities to communicate positively relates to developing and maintaining SMMs in selfmanaging teams (Druskat \& Pescosolido, 2002). This positive effect diminishes when organizational support decreases. The present results show that similar effects occur in actions teams of ATCOs. Future studies should reveal more details on how an organization can support team members in the development of SMMs, for example due to providing opportunities to exchange information and knowledge after task execution (Tannenbaum \& Cerasoli, 2013).

Interestingly, the results showed no differences in mental model similarity of the team-related SMM type between ATCOs of different organizations. ATCOs seems to have a similar understanding of teamwork irrespective of the workplace. The basic ATCO training provides guidelines for cooperation of ATCOs with each other and with pilots (ICAO, 2001). It is possible that these guidelines led to similar team-related SMMs among ATCOs of separate organizations. This would be an important and motivating insight as ATCOs of different organizations and countries will have to cooperate more frequently and intensively in the future (Langan-Fox et al., 2009; SESAR, 2012). A similar understanding of teamwork may ease the implementation of a "single European sky". 
The relationship between organizational context and ATCOs' SMM was mediated by ATCOs' work experience. ATCOs of separate organizations and with less than 10 years of experience differed in mental model similarity. This effect diminished for ATCOs with more than 10 years of work experience. This finding corroborates the idea that work experience is positively related to SMMs (Cooke et al., 2001; Levesque et al., 2001; Smith-Jentsch et al., 2001). With increasing experience with the task and interaction with others, team members' mental models become more similar. This finding also implies that particularly unexperienced team members may be sensitive to external circumstances, such as the organizational context, during the development of mature and shared knowledge structures (Druskat \& Pescosolido, 2002). They may integrate aspects inherent to the organization in their SMMs and since these aspects differ between separate organizations, ATCOs affiliated to these separate organizations differ in their SMMs.

\section{Limitations and future research}

This study may have some potential limitations that restrict the interpretation of the results. Participants included controllers working at radar positions but no tower controllers who control aircraft on sight. It is possible that tower controllers have different shared knowledge structures. For example, situational knowledge could be a distinctive SMM type in tower control as the task is more time critical. Our results are also limited by the use of pre-existing groups instead of randomly assigned groups. Consequently, groups may have differed systematically in other aspects than the organization and the level of work experience, such as age. However, as age and work experience correlated in this study, the influence of this variable should be little.

Another restriction of this study is that the closed card sorting task comprised predefined knowledge elements and categories. This may have limited the emergence of new elements and SMM types and thus prevented a complete overview of the ATCOs' knowledge. However, the selection was based on our research in Chapter 2 and card sorting has been described as an effective method to capture SMMs (DeChurch \& Mesmer-Magnus, 2010b).

Future research should also explore permutation test as potential analysis to study mental model similarity. The present study shows that permutation test is a valuable method to compare two groups consisting of many team members. We collected data of 100 ATCOs and the permutation test enables analysing specific group differences, e.g. as in this study, low and high levels of work experience.

Although we found differences in mental model similarity among ATCOs of separate organizations, it is unclear whether these affected performance. Note, that ATCOs at both organizations deliver safe and efficient ATC despite differences in mental model similarity. Further research should therefore study the SMM similarity with regard to team processes and performance. This study helps in giving directions to the SMMs types that should be included in such an analysis. 


\section{Chapter 3}

\section{Practical implications}

The present findings have some important practical implications. First, the approach applied can be used to assess SMMs within big teams. The combination of card sorting and permutation tests can help to identify SMM types that still show little similarity among team members and need additional attention in training sessions. Computer-based training (Smith-Jentsch et al., 2001) or cross-training (Marks, Sabella, Burke, \& Zaccaro, 2002), for example, help team members to develop a similar understanding of teamwork. In these training forms, team members increase their similarity of teamwork mental models through jointly categorizing effective and ineffective teamwork examples, and gaining hands-on task experience of each other's roles. This could be specifically interesting for unexperienced team members working for separate organizations. These teams often know little about how others carry out their tasks exactly at another organization and could therefore benefit from joint training sessions.

The identified dimensions of SMMs can also contribute to the effectiveness of training. The training of dynamic and transient knowledge requires different methods than the training of knowledge that is more static and possibly relatively easy integrated in existing knowledge structures (Kirschner \& Van Merriënboer, 2008). For example, mental models regarding equipment is quite static as it comprises procedural knowledge and remains unaltered in various task settings. For this type of knowledge both applies individual and team trainings early during the education programme. An alternative training method seems necessary for more dynamic and situation specific aspects of task procedures. Scenario-based training could be a successful training form because it offers a variety of situations in a realistic environment (Cannon-Bowers, Burns, Salas, \& Pruitt, 1998). The present results can guide training experts in choosing a suitable method for the training of different types of knowledge

Another implication relates to the plans in Europe to create a single European sky. This requires ATCOs of various air navigation service providers to closely work together (SESAR, 2012). This study furnishes evidence on an empirical basis that we cannot simply assume that these ATCOs share the same mental models. The culture, policies and people of the organization influence SMMs of unexperienced ATCOs (Uitdewilligen et al., 2010). It seems advisable to support this group of separate organizations in the development of SMMs that enable them to work together efficiently. Organizational support to develop SMMs could have several forms such as longer training session and more opportunities for interpersonal communication and information exchange (Druskat \& Pescosolido, 2002). Organizations should provide this support particularly to unexperienced team members and may decrease the support over time.

In conclusion, the results of the present research indicate that SMMs in teams should be analysed in terms of a number of separate and specific SMM types. The presents study applied a theory-driven approach and revealed domain-specific insights about the SMM types in teams of ATCOs of separate organizations and with different levels of work experience. It shows that research 
into domain-specific team cognition and shared knowledge representation is likely to increase theoretical insights and practical applications. 



\section{Chapter 4}

\section{Knowledge sharing facilitates a team's shared mental models, communication and performance}

In this study, we combine literature on knowledge sharing and shared mental models. We hypothesize that knowledge sharing improves a team's shared mental models and communication, and that communication further affects shared mental model development. We assume that both knowledge sharing and shared mental models correlate with team performance. Forty 3-person teams participated in either a knowledge sharing or a control condition and conducted a dynamic team task during three consecutive trials. Results show that knowledge sharing prior to task performance positively influenced mental model accuracy and the similarity of team-related mental models, but not taskrelated mental models. Team members in the knowledge sharing condition communicated more taskand team-related information during task performance, which in turn predicted mental model accuracy. Mental model accuracy was positively associated with team performance. Overall knowledge sharing improved team performance and especially in the final trial. We discuss the implications of knowledge sharing as an intervention to improve team members' shared mental models. 



\section{Introduction}

For a team to be effective, its members need to interact, communicate, and share knowledge efficiently (Brannick, Salas, \& Prince, 1997; DeChurch \& Mesmer-Magnus, 2010a, 2010b; Uitdewilligen et al., 2010). Especially the formation of shared mental models (SMMs) is crucial to successful team performance (DeChurch \& Mesmer-Magnus, 2010b). SMMs are common knowledge structures among team members about aspects of the task and team (Mathieu et al., 2010). The similarity and accuracy of these common knowledge structures jointly contribute to team effectiveness (Lim \& Klein, 2006). SMMs help team members to explain and interpret the task in a similar way, and have similar expectations of future task situations (Cannon-Bowers et al., 1993). Since SMMs play a significant role in team performance, this study addresses how SMMs develop and test whether knowledge sharing help teams in this development.

SMMs in a team presumable develop gradually and iterative (Kennedy \& McComb, 2010). Gradual development means that team members first share knowledge about aspects of the task and subsequently about aspects of the team. This phased development may result from the need to understand the task and responsibilities, before knowledge about other team members and interaction forms are meaningful (McComb, 2007). Iterative development of SMMs implies that individuals share knowledge repetitively until individual mental models converge to SMMs (Kennedy \& McComb, 2010). The convergence of individual mental models to SMMs involves interaction and knowledge exchange between team members (Langan-Fox et al., 2004; McComb, Kennedy, Perryman, Warner, \& Letsky, 2010). Despite these insights on mental model convergence, the exact underlying process of the formation of effective SMMs remains unclear and empirical evidence is missing.

The present study investigates the formation of effective SMMs by combining ideas from the knowledge sharing literature with contemporary notions on SMM development. Knowledge sharing is the process through which individuals mutually exchange their knowledge (De Vries \& Van den Hooff, 2006; Mesmer-Magnus \& DeChurch, 2009). While knowledge sharing and SMMs are conceptually related, few studies have tested their relationship (e.g. Gross \& Kluge, 2014).

The main objective of this paper is to investigate whether knowledge sharing positively predicts SMMs. This investigation increases the understanding about the development of SMMs and contributes to team training practices by discussing the effectiveness of knowledge sharing in improving SMMs among team members. According to the gradual approach of SMM development (Kennedy \& McComb, 2010; McComb, 2007), we guide team members in knowledge sharing of first task aspects and then team aspects. Moreover, we suggest that knowledge sharing results in more communication of relevant SMM content during task performance as team members tend to talk more about shared than unshared knowledge (Stasser \& Titus, 1985; Stasser \& Titus, 1987). This repetitive exchange of knowledge through knowledge sharing and communication should positively affect team 


\section{Chapter 4}

members SMMs (Kennedy \& McComb, 2010). Finally, we suppose that the positive effects of knowledge sharing eventually lead to better team performance.

\section{Shared Mental Models in Teams}

SMMs in teams are essential to operate effectively (DeChurch \& Mesmer-Magnus, 2010a). They enable team members to have accurate expectations about the situation and coordinate their actions successfully (Cannon-Bowers et al., 1993). Team members with effective SMMs anticipate each other's intentions and behaviour and have a similar understanding of the task (Langan-Fox et al., 2004; Waller et al., 2004). Two distinctive properties of SMMs are similarity and accuracy (Rentsch et al., 2008; Resick et al., 2010). Mental model similarity refers to the compatibility of knowledge structures between team members, whereas mental model accuracy describes how well knowledge structures represent the real world (Lim \& Klein, 2006).

Team members develop multiple types of SMMs referring to different aspects of the task domain (Cannon-Bowers et al., 1993). These SMMs can be distinguished in two broader categories, namely SMMs pertaining to the task and SMMs pertaining to the team (Mathieu et al., 2000). Taskrelated SMMs include knowledge about task rules, strategies, equipment and environmental influences. Team-related SMMs include knowledge about competences of other team members, interaction patterns and task interdependencies. Task-related and team-related SMMs both contribute to successful team performance (Mathieu et al., 2010), although the strength of the relationship varies with different task and team contexts (Lim \& Klein, 2006; Mathieu et al., 2000; Smith-Jentsch et al., 2005). We include both task-related and team-related SMMs in this study, as successful performance in the present task context requires common knowledge about task and team. To confirm this main idea on the relationship between SMMs and team performance, the present study test the following hypothesis:

Hypothesis 1. Task- and team-related SMMs (similarity and accuracy) are positively related to team performance.

\section{Development of Shared Mental Models}

The differentiation into task-related and team-related SMMs plays also a role in the development of SMMs. SMMs are believed to develop gradually as task-related SMMs develop before team-related SMMs (McComb, 2007). The reason for this gradual development lies in the assumption that teamrelated SMMs become not effective until team members have developed SMMs of task aspects (Kennedy \& McComb, 2010). These task-related SMMs explain the various ways of how to conduct the task and this may be necessary to understand the role of different team members in this task, which is knowledge included in team-related SMMs. The gradual development may explain why performance of fresh-starting teams often stronger relates to SMMs on task aspects than to SMMs pertaining to the team (Banks \& Millward, 2007; Mathieu et al., 2005). 
SMMs also develop more effectively if team members repetitively exchange their individual mental models (Kennedy \& McComb, 2010). This iterative process is driven through communication of knowledge essential for team functioning and continues until individual mental models converge into SMMs. Once individual mental models have converged into SMMs, team members need to update and adapt their SMMs to changing task situations (Burke et al., 2006; Uitdewilligen et al., 2013). The repetitive examination of knowledge available in the team is thus important for the development and maintenance of effective SMMs (McComb et al., 2010).

Despite these theoretical ideas on the gradual and iterative development of SMMs, we know little about the underlying processes. This is important to support team members in the process of effective SMM development and improve team performance. A possible process to explain the development of SMMs is knowledge sharing.

\section{The Role of Knowledge Sharing in Shared Mental Models}

Knowledge sharing is the process through which team members exchange relevant knowledge, information and expertise with each other (De Vries \& Van den Hooff, 2006; Mesmer-Magnus \& DeChurch, 2009). During knowledge sharing, team members provide and receive explicit and tacit knowledge through, for example, communication, observation and apprenticeship (Bartol \& Srivastava, 2002). Knowledge sharing positively influence various team outcomes, such as team cohesion and satisfaction (Mesmer-Magnus \& DeChurch, 2009), but we know little about its influence on SMMs.

This study suggest that knowledge sharing positively affects team members' SMMs. More precisely, we suggest that knowledge sharing is an effective process (Mesmer-Magnus \& DeChurch, 2009), which can lead to a common cognitive state among team members, namely their SMMs (Marks et al., 2001). Team members who engage in knowledge sharing create a joint understanding of previously individual knowledge (De Vries \& Van den Hooff, 2006) and consequently should develop more similar and accurate mental models (Cannon-Bowers et al., 1993). Their individual mental models converge to mental models, which are shared among team members.

A few studies support the idea that knowledge sharing and SMMs are positively related. These studies (Gross \& Kluge, 2012, 2014) showed that knowledge sharing in steel mill workers had impact on multiple types of SMMs related to equipment, task, team and interaction. The researchers also found a trend for a positive correlation between knowledge sharing and mental model similarity of steel mill workers (Gross \& Kluge, 2014). Teams of workers that scored high on knowledge sharing, also scored high on mental model similarity. On the other hand, teams that scored low on knowledge sharing also scored low on mental model similarity. While these results give reason to assume that knowledge sharing and SMMs are positively related, the results lack statistical significance. Moreover, the research described does not differentiate between mental model similarity of task-related and team- 


\section{Chapter 4}

related aspects, nor does it address the relationship between knowledge sharing and mental model accuracy.

The present study contributes to existing literature through testing the effects of knowledge sharing on the similarity of task- and team-related mental models and on mental model accuracy. To test this idea, half of the teams in this study participate in a knowledge sharing condition and the other half in a control condition. Such a design can be effective in showing the impact on knowledge sharing on SMMs (Sikorski, Johnson, \& Ruscher, 2012). In line with ideas on the gradual development of SMMs (Kennedy \& McComb, 2010; McComb, 2007), we guide team members in first knowledge sharing of task aspects and subsequently team aspects. This approach aims to support the development of task-related SMMs before team-related SMMs.

Hypothesis 2. Team members develop more task- and team-related SMMs (similarity and accuracy) when they have engaged in knowledge sharing of aspects of the task and the team.

\section{The Role of Knowledge Sharing in Communication}

This study assumes that team members communicate more information during task performance after they have engage in knowledge sharing. This assumption is based on a common phenomenon in knowledge sharing, namely that teams communicate more about shared than unshared information (e.g. Greitemeyer, Schulz-Hardt, Brodbeck, \& Frey, 2006; Mesmer-Magnus \& DeChurch, 2009). The information sampling bias (Stasser \& Titus, 1985; Stasser \& Titus, 1987) explains this phenomenon in more detail. In many team tasks, information is distributed among various team members. Although team members could benefit from communicating this information they rarely do so (Mesmer-Magnus \& DeChurch, 2009). Instead, they are biased towards the information already shared with other team members (Stasser \& Titus, 1987). This is because the more team members know a piece of information, the more likely that one of them will bring it up during discussion. If only one team member knows a piece of information, only this team member can contribute the information to the discussion. The probability that the team communicates about this unshared piece of information significantly decreases.

The information sampling bias contradicts ideas in SMM research that assume that team members with effective SMMs easily coordinate their actions without extensive verbal communication (E. L. Blickensderfer, Reynolds, Salas, \& Cannon-Bowers, 2010; Cannon-Bowers et al., 1993). Instead, they coordinate implicitly as they have similar expectations of the task and anticipate each other's needs and behaviour (Espinosa, Lerch, \& Kraut, 2004; Mohammed et al., 2010). Such a positive effect of SMMs on coordination would imply that team members that developed effective SMMs through knowledge sharing communicate less during subsequent task performance (LanganFox et al., 2004).

Despite this implication, this study suggests that team members in the knowledge sharing condition communicate more than team members in the control condition. This increase in 
communication occurs for two reasons. First, teams in this study are fresh-starting teams. It is unlikely that, in the short period of the experimental session, these fresh-starting teams develop SMMs that are so effective that they allow implicit coordination (Mohammed et al., 2010). Second, in fresh-starting teams that perform a new task (as it is the case in the present study) most information is initially unshared between team members. This initial information distribution is a strong predictor of the information sampling bias (Mesmer-Magnus \& DeChurch, 2009). That is, team members communicate more about shared information than unshared information, specifically if information is initially unshared.

In sum, we assume that initially unshared information converges to shared information through knowledge sharing. Following the reasoning that team members communicate more about shared information than unshared information, we would expect that team members in the knowledge sharing condition have more to talk about in subsequent task performance. Specifically, the probability increases that team members communicate task-related information after they engage in knowledge sharing regarding aspects of the task. Similar, they communicate more team-related information after knowledge sharing of team aspects. Team members in the control condition have more unshared than shared information available and should consequently communicate less. The following hypothesis tests this assumption:

Hypothesis 3. Team members communicate more task-related information after they have engaged in knowledge sharing of aspects of the task and more team-related information after they engage in knowledge sharing of aspects of the team.

\section{The Role of Communication in Shared Mental Models}

The expected increase in communication of relevant SMM content should further reinforces SMMs of team members in the knowledge sharing condition. This is because SMMs develop iteratively (McComb, 2007). The idea is that team members in the knowledge sharing condition first examine relevant information during knowledge sharing and subsequently again during communication. This repetitive examination of relevant information should help team members to develop SMMs that are more effective (Kennedy \& McComb, 2010; McComb et al., 2010). Specifically, it increases the likelihood that individual knowledge becomes shared among team members and integrated in the team's SMMs.

The second reason assuming a positive relationship between communication and SMMs is because communication among team members itself is essential to the development of more similar and accurate mental models (Andres, 2012; McComb, 2007; Waller et al., 2004). For example, communication of the team's goal, the course of action and roles and responsibilities of other team members contributes to the emergence and maintenance of SMMs (Burke et al., 2006). Particularly, if members of newly formed teams need to align their individual mental models, exchanging information through communication is crucial (McComb, 2007; Zijlstra et al., 2012). According to the iterative 


\section{Chapter 4}

nature of SMM development and the relationship of SMMs and communication, we formulate the following hypothesis:

Hypothesis 4. Communication of task- and team-related information positively affects taskand team-related SMMs (similarity and accuracy).

\section{Effects on Team Performance}

Finally, if knowledge sharing facilitates the development of SMMs and enhances communication among team members, we can expect a positive effect on team performance. Various studies have proven the positive effects of knowledge sharing on the team-level and the organizational-level. For example, effective knowledge sharing results in successful task performance, team cohesion and team member satisfaction (Mesmer-Magnus \& DeChurch, 2009; Wang \& Noe, 2010). Moreover, knowledge sharing positively predicts firm performance, innovation capabilities and cost reduction (for a review see Wang \& Noe, 2010).

In the present study, we expand this idea by investigating how guided knowledge sharing that intends to improve the development of team members' SMM relate to team performance. Knowledge sharing in the present study first deals with task aspects and later focuses on team aspects to support the gradual development of SMMs. This leads to the final hypothesis:

Hypothesis 5. Team members perform better when they engage in knowledge sharing of aspects of the task and the team.

Figure 4.1 illustrates the research model of this study. In sum, we assume that knowledge sharing has a positive effect on communication among team members, their SMMs and team performance. Additionally, we expect that increased communication improves SMMs and SMMs in turn positively predict team performance.

H5

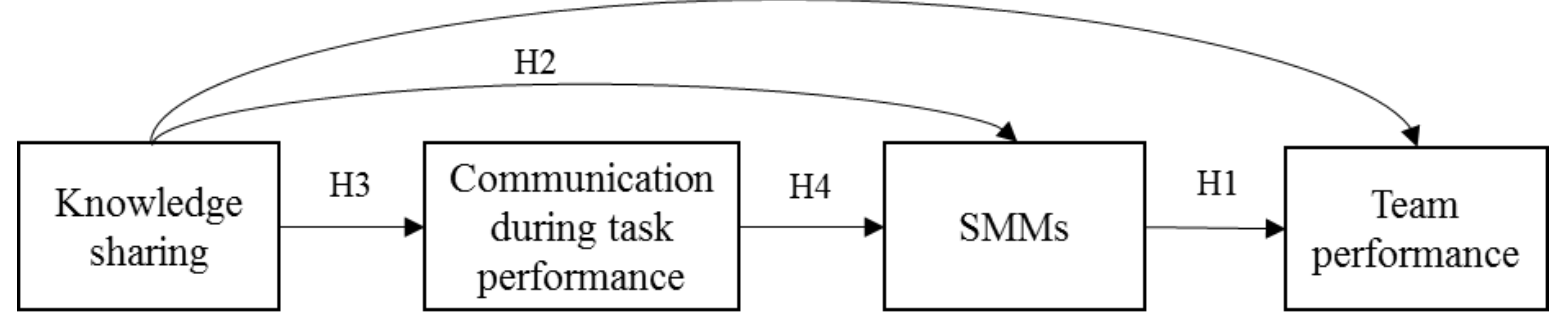

Figure 4.1. The research model. 


\section{Method}

To test the hypotheses we conducted an experiment with an experimental and a control conditions. In the experimental condition team members were guided in knowledge sharing of first task aspects and then team aspects before they performed a team task. In the control condition, team members performed the team task without the opportunity for knowledge sharing.

\section{Participants}

A total of 120 undergraduate students ( 84 were female) formed 40 three-person teams and participated for course credits. Twelve teams were all female and five were all male. There was no effect of gender composition on team performance, $\mathrm{F}(2,37)=.30, \mathrm{p}=.74 ; \mathrm{F}(2,37)=.97, \mathrm{p}=.39$. Participants' mean age was 20.9 years ( $\mathrm{SD}=3.3$ years). They gave prior informed consent as approved by the faculty's research ethics committee.

\section{Task}

For the purpose of this study, we used a dynamic team environment called TeamTris that has successfully been applied previously (Sander et al., 2015). TeamTris is a PC-based, cooperative version of the well-known Tetris ${ }^{\mathrm{TM}}$ game that provides good experimental control. Several teamcritical aspects that were inspired by air traffic control were integrated in TeamTris and enable generalisation to dynamic team environments. These include role interdependency, the potential benefits of information exchange and the application of strategies, and the clear need for planning behaviour and situation awareness. Moreover, TeamTris provides a dynamic task environment to study team cognition and behaviour as required in non-static environments (Van der Pal \& Justen, 2011). In comparison with other synthetic tasks used in SMM research, TeamTris requires little practice, but it needs time and effort of a team to fully master the game. As such, TeamTris provides a cooperative game environment suited for fresh-starting teams with rudimentary knowledge about the task, while fellow team members have not met before.

In TeamTris, three participants work together as either 'Planner', 'Controller 1', or 'Controller 2', and have to manipulate game pieces effectively in a shared game interface. Participants sit individually behind a personal computer that is part of a local intranet. Every participant sees the entire TeamTris environment including the actions of the other players. Verbal communication among participants during task execution is possible via connected headsets. Identical to regular Tetris ${ }^{\mathrm{TM}}$, the team's task is to position game pieces to complete lines of blocks at the bottom of the game field. Planner and controllers fulfil different roles to reach the team goal. The Planner selects appropriate game pieces and positions them at the top of the game field of Controller 1 and 2. The Planner may also use an alternative task strategy and decide to place a game piece on hold for later assignment to a controller. If the Planner fails to assign a game piece in time, i.e. before a controller has positioned the last game piece on the bottom of the game field, the system will assign a random game piece. Both 


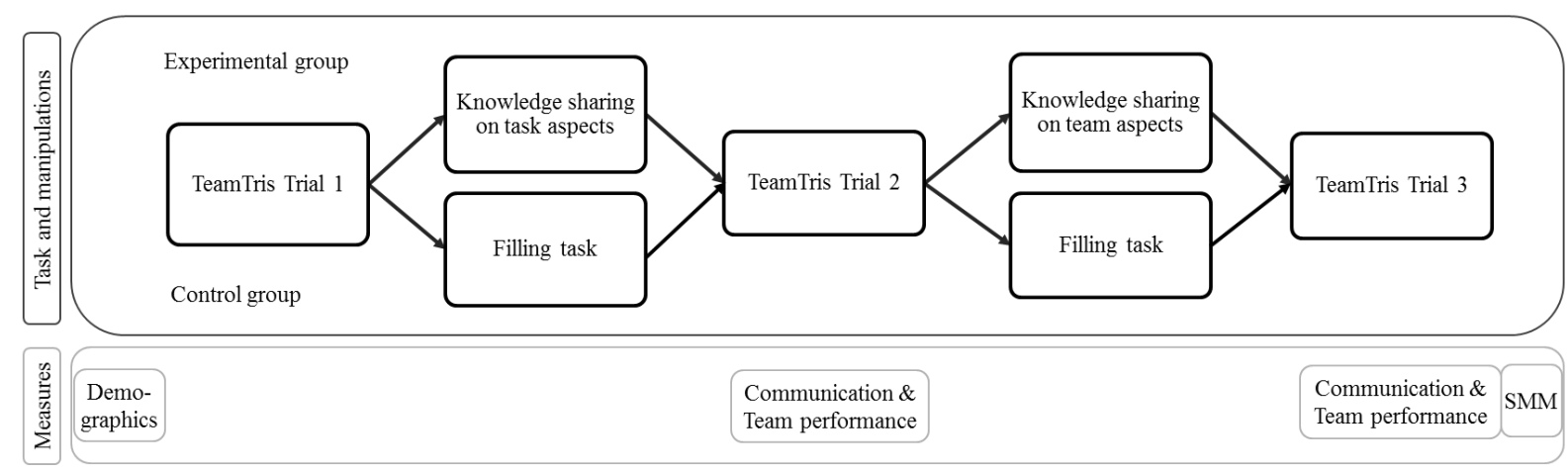

Figure 4.2. Schematic representation of the time line of the experimental session

controllers are responsible for positioning each game piece as it moves downward through their individual game areas to a suitable spot on the bottom of the screen or piled on previously positioned game pieces. In the best of cases, all game pieces connect into an uninterrupted horizontal line of blocks. One or more complete block lines across the games areas of both controllers will disappear and this will yield points. The team's goal is to clear as many block lines as possible within a given period of time.

Participants need to work together closely, because their limited access to their individual part of the interface allows little role overlap. To further increase interdependency among participants, TeamTris was designed to comprise a number of cooperative game elements. The four most important elements are: (a) penalty points are assigned when the system instead of the Planner selects and assigns a game piece, (b) bonus points are assigned when four block lines are cleared simultaneously, (c) controllers can exchange game pieces, and (d) some game pieces cannot be rotated by either Controller 1, Controller 2, or by both. In general, effective team performance depends on the team members' ability to adopt a strategy that fits their individual competencies but also considers mutual role interdependencies.

\section{Procedure}

Upon arrival, participants were informed that the session would take about 2 hours, and they were randomly assigned to one of the three team roles (Planner, Controller 1, or Controller 2) of a 3-person team. Half of the teams were assigned to the knowledge sharing condition and the other half formed the control group. Participants were first seated at an individual PC, and then filled in a demographic pre-questionnaire. They were instructed only to talk to each other during game play or when otherwise instructed. Figure 4.2 illustrates a typical experimental session.

Participants were instructed to read a description of their task. This contained specific information on the role they were assigned to and unique information that was relevant for successful team performance. For example, only the Planner's description stated that penalty points would be assigned if no shape was planned in on time. Most performance relevant information was therefore initially unshared (see Table 4.1 for an overview of which information was initially known by either 
the Planner, Controller 1 or/and Controller 2). The team then played TeamTris for 10 minutes. During game play, participants were free to communicate and all communication was recorded. This first baseline trial (trial 1) was designed for familiarisation with the task and to gain necessary experience. During this first trial, participants were still able to rotate all game pieces. The two subsequent 10 minutes TeamTris trials (trial 2 and trial 3) were more challenging as controllers could no longer rotate some of the game pieces. These trials were each preceded by 5 minutes guided knowledge sharing or by compensatory tasks in the control condition. After trial 3, all participants completed the SMM assessment.

Knowledge sharing manipulation. The knowledge sharing manipulation was designed according to principles of cross-training as a general strategy to guide knowledge sharing (Bartol \& Srivastava, 2002; Sikorski et al., 2012; Volpe, Cannon-Bowers, Salas, \& Spector, 1996). Knowledge sharing consisted of two subsequent manipulations types. These were knowledge sharing on task aspects prior to trial 2 and knowledge sharing on team aspects prior to trial 3. Both manipulations lasted five minutes.

During task-related knowledge sharing, designed according to positional clarification (E. Blickensderfer, Cannon-Bowers, \& Salas, 1998), participants reread their own task description and reviewed each other's task descriptions. They were asked to carefully read all descriptions on their pc screen and make sure they fully understand their own and other's tasks. This manipulation was meant to increase the exchange of unshared information on task aspects and thus stimulate the development of task-related SMMs.

In the second knowledge sharing manipulation, participants exchanged information during a team discussion (Gorman, Cooke, \& Amazeen, 2010). Team members sat down around a table and discussed five team-related questions listed on a checklist. These questions included: (1) How does your task contribute to the overall task? (2) How well do you communicate as a team? Can you improve your communication? (3) Do you work together effectively? How can you improve teamwork? (4) What is your team strategy? (5) How can you distribute the workload among each other? The goal of this discussion was actively exchanging unshared information about aspects of the team thereby helping team members to develop team-related SMMs.

The compensatory tasks of the teams in the control condition also lasted five minutes but focused solely on examination of individual unshared knowledge. Preceding to TeamTris trial 2, the task description of the corresponding role was presented on the pc screen. Participant were asked to carefully reread their own task description to ensure that they fully understand their task. Proceeding to trial 3, participants were asked to write an instruction of their own task on their pc for someone who is unfamiliar with the task. Both compensatory tasks intended to keep participants in the control condition busy with TeamTris for the same amount of time as participants in the knowledge sharing condition. 


\section{Chapter 4}

\section{Measures}

Team performance. Team performance was the total team score per TeamTris trial (trial 1 - 3). Note, that practice trial 1 was easier than trial 2 and 3, which were both equally difficult. The score per trial was composed of rewarded points (cleared block lines) and assigned penalty points when the Planner failed to timely assign a game piece to one of the controllers, or when to game pieces collided in the area of one of the controllers. Rewards, penalties and their summation were automatically logged.

Communication of task- and team-related information. To assess communication of task- and team-related information, all verbal communication of participants during task execution was recorded and transcribed per game trial. A trained rater then coded whether participants communicated information of task and team aspects that were initially unshared but relevant for team performance (see Table 4.1 for descriptions and examples of the various coding items and the distribution of information among the different roles (Planner, Controller 1 or/and Controller 2)). Initially unshared task-related information included seven items (collision, holding, non-planning, rotation, space button, TeamTris and whole field). For example, only Controller 1 knew that penalty points were assigned for the collision of two game pieces. If participants discussed this information during game play in the team, the rater marked the item 'collision' as communicated. Scores for communication of task-related information varied between 0 (no item discussed) and 7 (all items discussed). Initially unshared teamrelated information included six items (labelling, name, score, slow-down, time, and transfer of control). Scores for communication of team-related information varied between 0 (no item discussed) and 6 (all items discussed).

To ensure scoring reliability, $45 \%$ of the teams $(n=18)$ were rated by two individuals who were pre-trained to score communication transcripts on the items described. Cohen's Kappa (Cohen, 1960) yielded the inter-rater reliability scores for task-relevant $(\kappa=.72)$ and team-relevant communication $(\kappa=.63)$. It is not surprising that inter-rater reliability for team-related information was slightly lower since it is more abstract and contains less factual content. As both reliability scores were above .60, substantial strength of agreement was concluded (Landis \& Koch, 1977).

Mental model similarity. The mental model similarity measure applied a successfully used approach (Mathieu et al., 2010; Smith-Jentsch et al., 2005). It assumes a consistency index rather than a consensus index and entails that individuals share compatible yet not identical mental models. In TeamTris several alternative actions can be effective in solving a problem and it is important that individuals have similar perceptions of the relative value and not the absolute value of these actions to problem solving during the game (Mathieu et al., 2010). 
Table 4.1

Description and examples of communication coding.

\begin{tabular}{|c|c|c|c|c|}
\hline $\begin{array}{l}\text { Type of } \\
\text { information }\end{array}$ & $\begin{array}{l}\text { Coding } \\
\text { item }\end{array}$ & Description & $\begin{array}{l}\text { Known } \\
\text { by }\end{array}$ & Example \\
\hline \multirow[t]{7}{*}{$\begin{array}{l}\text { Task-related } \\
\text { information }\end{array}$} & Collision & $\begin{array}{l}\text { Penalty points are assigned if game } \\
\text { pieces collide }\end{array}$ & $\mathrm{C} 1$ & $\begin{array}{l}\text { "Watch out! We get minus points } \\
\text { when blocks crash." }\end{array}$ \\
\hline & Holding & $\begin{array}{l}\text { The planner can 'park' game pieces } \\
\text { in the holding for later use }\end{array}$ & PL & $\begin{array}{l}\text { "I'll put this shape in the holding and } \\
\text { give it later to you." }\end{array}$ \\
\hline & $\begin{array}{l}\text { Non- } \\
\text { planning }\end{array}$ & $\begin{array}{l}\text { Penalty points are assigned if the } \\
\text { planner have not planned in the } \\
\text { following game piece on time }\end{array}$ & PL & $\begin{array}{l}\text { "Guys, we get penalty points when I } \\
\text { have no time to plan the next shape } \\
\text { for you." }\end{array}$ \\
\hline & Rotation & $\begin{array}{l}\text { The controllers cannot rotate three } \\
\text { shapes: s-shape (pink), z-shape } \\
\text { (orange) and t-shape (brown) }\end{array}$ & $\begin{array}{l}\mathrm{C} 1 \\
\mathrm{C} 2\end{array}$ & $\begin{array}{l}\text { "No! Not the brown one. I cannot } \\
\text { move it." }\end{array}$ \\
\hline & $\begin{array}{l}\text { Space } \\
\text { button }\end{array}$ & $\begin{array}{l}\text { The space button allows to switch } \\
\text { control from one game piece to } \\
\text { another }\end{array}$ & $\begin{array}{l}\mathrm{C} 1 \\
\mathrm{C} 2\end{array}$ & "Use the space button, mate. Quick!" \\
\hline & TeamTris & $\begin{array}{l}\text { Most reward points are assigned if } \\
4 \text { lines a cleared simultaneously, } \\
\text { called a TeamTris }\end{array}$ & $\mathrm{C} 2$ & $\begin{array}{l}\text { "We should build four lines on top of } \\
\text { each other. This earns the most } \\
\text { points." }\end{array}$ \\
\hline & $\begin{array}{l}\text { Whole } \\
\text { field }\end{array}$ & $\begin{array}{l}\text { The game pieces must cover the } \\
\text { whole game field without holes }\end{array}$ & $\begin{array}{l}\mathrm{C} 1 \\
\mathrm{C} 2\end{array}$ & $\begin{array}{l}\text { Oh no, the line does not disappear } \\
\text { because game pieces do not cover } \\
\text { the whole field." }\end{array}$ \\
\hline \multirow[t]{6}{*}{$\begin{array}{l}\text { Team- } \\
\text { related } \\
\text { information }\end{array}$} & Labelling & $\begin{array}{l}\text { When the planner assigns a game } \\
\text { piece to a controller, a label } \\
\text { appears next to the game piece. }\end{array}$ & PL & $\begin{array}{l}\text { Controller 1: "Which one do I get } \\
\text { next?" - Planner: "The green one. } \\
\text { The label tells you." }\end{array}$ \\
\hline & Name & $\begin{array}{l}\text { Knowing the names of team } \\
\text { members helps concise } \\
\text { communication }\end{array}$ & $\mathrm{C} 1$ & "What is your name?" \\
\hline & Score & $\begin{array}{l}\text { The planner is in charge of } \\
\text { informing other team members } \\
\text { about the score }\end{array}$ & PL & $\begin{array}{l}\text { "We're doing well, we have } 97 \\
\text { points." }\end{array}$ \\
\hline & $\begin{array}{l}\text { Slow } \\
\text { down }\end{array}$ & $\begin{array}{l}\text { Controllers should slow down their } \\
\text { actions if the planner has too little } \\
\text { time to assign game pieces }\end{array}$ & PL & $\begin{array}{l}\text { "Don't move to quick. I have no time } \\
\text { to plan in the next shape" }\end{array}$ \\
\hline & Time & $\begin{array}{l}\text { Controller } 2 \text { is in charge of } \\
\text { keeping track of the remaining } \\
\text { time }\end{array}$ & $\mathrm{C} 2$ & $\begin{array}{l}\text { "I'll tell you when we have one } \\
\text { minutes left." }\end{array}$ \\
\hline & $\begin{array}{l}\text { Transfer } \\
\text { of control }\end{array}$ & $\begin{array}{l}\text { A controller can transfer control of } \\
\text { a game piece to the other } \\
\text { controller by moving it across the } \\
\text { middle line }\end{array}$ & $\begin{array}{l}\mathrm{C} 1 \\
\mathrm{C} 2\end{array}$ & $\begin{array}{l}\text { "Give me the green one. I'll move it } \\
\text { over to her." }\end{array}$ \\
\hline
\end{tabular}

Note. $\mathrm{PL}=$ planner. $\mathrm{C} 1=$ controller $1 . \mathrm{C} 2=$ controller 2. 
Task-related SMMs were assessed using the so-called cue-strategy association measure (Smith-Jentsch et al., 2005). We designed four scenarios each depicting a possible situation in TeamTris, and each with four possible actions. Participants rated the desirability of applying each action on a six-point scale ranging from 1 (very undesirable) to 6 (very desirable). The similarity score of task-related mental models was represented by the inter-member correlation between these ratings within each team (Mathieu et al., 2010).

Team-related SMMs were assessed using the positional goal interdependency measure (SmithJentsch et al., 2005). We developed four scenarios that described a possible course of action of the Planner or of one of the controllers. Participants then rated how this action would affect each role on a 7-point scale ranging from 1 (strong negative influence) to 7 (strong positive influence). Similarity of team-related mental models was represented by the inter-member correlation (Mathieu et al., 2010) between these ratings within each team.

Mental model accuracy. Team members' mental model accuracy was assessed via six statements regarding task rules and inter-positional knowledge, i.e. knowledge about other team members' position. Participants rated whether a statement was right or wrong, or whether they did not know the answer. The approach used to compute mental model accuracy was similar to the one used by Cooke and colleagues (2003). First, we computed mental model accuracy of each individual. Individual mental model accuracy was the proportion of correctly answered responses and 1 minus the proportion of items answered with "I don't know". The sum of these two proportions was divided by two. Equation 1 describes this computation with A(i) being the accuracy of person i, Ci is the number of corrected answers given by person i, Ui is the number of unknown ("I don't know") answers given by person $\mathrm{i}$, and $\mathrm{T}$ is the total number of possible answer.

$$
A(i)=\frac{\frac{C_{i}}{T}+\left(1-\frac{U_{i}}{T}\right)}{2}
$$

Mental model accuracy of a team was then computed by averaging the individual mental model accuracy scores per team, yielding scores ranging from 0 (no accuracy) to 1 (complete accuracy).

\section{Aggregation of individual measures}

SMM indices were based on averaging individual responses, while they represent a higher-order construct at the team level. This aggregation of individual responses to the team level must be justified by establishing sufficient systematic variance in the individual responses. We tested variances in individual responses using ANOVAs (Bliese, 2000; Mathieu et al., 2010). For task-related mental model similarity, we conducted a within-subject 4 (scenario) x 4 (action) ANOVA, which revealed significant main effects for scenario, $F(2,238)=67.34, p<.01$, and action, $F(2,238)=63.18, p<.01$. Also the interaction effect for scenario by action was significant, $F(4,476)=41.61, p<.01$. These 
results imply that participants perceived differences between scenarios, that some actions were in general more desirable than others, and that some actions were more desirable than others given a specific scenario. This was a reliable basis to compute the inter-member correlations for task-related mental model similarity within each team. These ranged from $r=-.09$ to $r=.84(M=.47, S D=.24)$.

For team-related mental model similarity, we performed a within-subject 4 (scenario) x 3 (role) ANOVA. We found significant main effects for scenario, $F(3,357)=67.92, p<.01$, and role, $F(3,357)=335.96, p<.01$. Furthermore, the interaction between scenario by role was significant, $F(9,1071)=48.88, p<.01$. These results imply that participants perceived that scenarios have different effects on all roles, that some roles were generally more influenced than others and that the four scenarios had different impacts on the different roles. Again, the results indicate sufficient variance between ratings so that we computed the inter-member correlations for team-related mental model similarity within each team. These ranged from $r=-.24$ to $r=.83(M=.50, S D=.23)$.

To justify the aggregation of mental model accuracy to the team level, we estimated the two intra-class correlation coefficients (ICC; Bliese, 2000). The ICC(1) indicates whether team members are interchangeable and implies sharing of a team-level construct measured at the individual-level. ICC(2) is a reliability index that provides evidence for the stability of a group mean. Analysis yielded an ICC(1) of .25 and an ICC(2) of .50. On the basis of these reliability estimates, we averaged individuals' accuracy scores per team (Bliese, 2000).

\section{Results}

\section{Relationship of Shared Mental Model and Team Performance}

To address whether SMMs were positively associated with team performance (hypothesis 1) we computed Pearson's correlations. Table 4.2 presents descriptive statistics and correlation coefficients of the study variables. Of interest for hypothesis 1 are correlations between SMMs and team performance at trial 2 and trial 3 as trial 1 only served as familiarization. Hypothesis 1 was partly confirmed since mental model accuracy significantly correlated with team performance at trial 2 and trial 3. The correlation between team performance and task- and team-related mental model similarity was not statistically confirmed in either trial.

\section{Effects of Knowledge Sharing on Shared Mental Models}

To test whether team members develop more task- and team-related SMMs when they engage in knowledge sharing (hypothesis 2), we ran independent t-tests. Table 4.3 represents average SMM values as a function of knowledge sharing and the results of the independent $t$-test. Task-related mental model similarity did not differ between conditions. Team-related mental model similarity and mental model accuracy were significantly higher in in the knowledge sharing condition than in the control condition. In all, knowledge sharing on task and team aspects led to more similar mental models of team aspects and to SMMs that were more accurate. 
Chapter 4

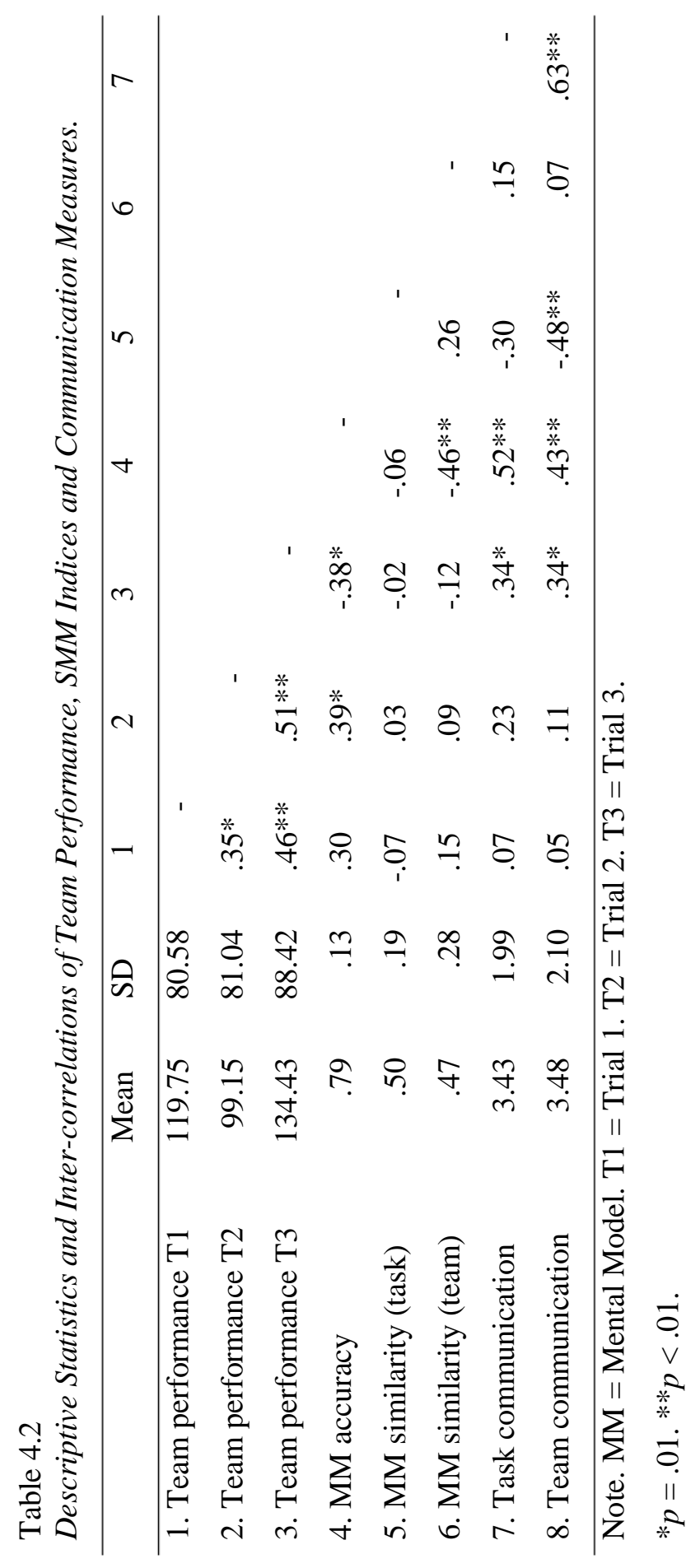


Table 4.3

Effects of Knowledge Sharing on SMMs.

\begin{tabular}{|c|c|c|c|}
\hline & \multicolumn{2}{|c|}{ Mental model similarity } & \multirow[t]{2}{*}{ Mental model accuracy } \\
\hline & Task-related SMM & Team-related SMM & \\
\hline & Mean $(S D)$ & Mean $(S D)$ & Mean $(S D)$ \\
\hline Knowledge sharing & $.50(.04)$ & $.56(.06)$ & $.88(.02)$ \\
\hline Control & $.49(.04)$ & $.37(.06)$ & $.70(.03)$ \\
\hline$t(\mathrm{df})$ & $.12(38)$ & $2.15(38)^{*}$ & $5.64(38)^{* *}$ \\
\hline
\end{tabular}

Note. $\mathrm{MM}=$ Mental Model.

$* p<.05 . * * p<.01$.

\section{Effects of Knowledge Sharing on Communication}

Table 4.4 provides an overview of communication of task-related information in trial 2 and communication of team-related information in trial 3 in the two conditions. Team members in the knowledge sharing condition communicated both more task-related information and more team-related information during task performance than team members in the control condition. These differences were statistically confirmed by separate independent sample t-test (Table 4.4). These outcomes support hypothesis 4 that team members engaging in knowledge sharing of relevant information about the task and the team positively affected team members' communication of this information during subsequent game play.

Table 4.4

Effects of Knowledge Sharing on Communication in the Subsequent Trial.

\begin{tabular}{lccc}
\hline Knowledge sharing & & $\begin{array}{c}\text { Number of task-related } \\
\text { communication items }{ }^{\mathrm{a}} \text { in T2 }\end{array}$ & $\begin{array}{c}\text { Number of team-related } \\
\text { communication items }^{\mathrm{b}} \text { in T3 }\end{array}$ \\
\cline { 3 - 4 } & & Mean $(S D)$ & Mean $(S D)$ \\
\hline Knowledge sharing & No & $2.7(1.8)$ & \\
on task aspects & Yes & $4.2(2.0)$ & $2.9(2.3)$ \\
Knowledge sharing & No & & $4.1(1.8)$ \\
on team aspects & Yes & & $2.73(38)^{* *}$ \\
\hline$t$ (df) & & $3.17(38)^{* *}$ &
\end{tabular}

${ }^{\mathrm{a}}$ the total number of items were $7 .^{\mathrm{b}}$ the total number of items were 6.

Note. $\mathrm{T} 2=$ trial $2 . \mathrm{T} 3=$ trial 3

$* * p>.01$.

\section{Effects of Communication on Shared Mental Models}

Table 4.1 provides an overview of the descriptive statistics and the inter-correlations between SMMs and communication measures (hypothesis 4). Results show that communication of task- and team- 


\section{Chapter 4}

related information correlated positively with mental model accuracy. Team-related communication negatively correlated with task-related mental model similarity. We found no correlation between communication and team-related mental model similarity.

Multiple regression analyses were conducted to test whether communication predicted SMM measures. Table 4.5 shows the results of the regression analyses. Communication of task-related information, but not team-related information, predicted mental model accuracy. In addition, communication of team-related information negatively explains a significant amount of the variance of task-related mental model similarity implying that more team-related communication resulted in less similar task-related mental models. Communication did not explain variance in team-related mental model similarity.

\section{Effects on Team Performance}

A repeated measures ANOVA with a mixed design, trial (1-3) by knowledge sharing condition (no, yes), was conducted to test the effect of knowledge sharing on team performance (hypothesis 5). Trial functioned as a within-subject factor whereas knowledge sharing entered the analysis as a betweensubject factor.

There was a significant main effect of trial, $F(2,76)=3.44, p<.05, \eta^{2}=.08$. A quadratic contrast provided a better fit of the factor trial, $F(2,76)=5.39, p=.02, \eta^{2}=.12$. There was also a significant interaction effect between trial and condition, $F(2,76)=3.33, p<.05, \eta^{2}=.08$. This indicates that team performance was different for the knowledge sharing and the control condition. Figure 4.3 suggests that the performance difference between conditions appeared after trial 2. Paired comparisons (Bonferroni correction applied) revealed that the increase in performance from trial 2 to 3 was significant $(p<.05)$. Differences in team performance became visible in trial 3, providing support for the final hypothesis. 


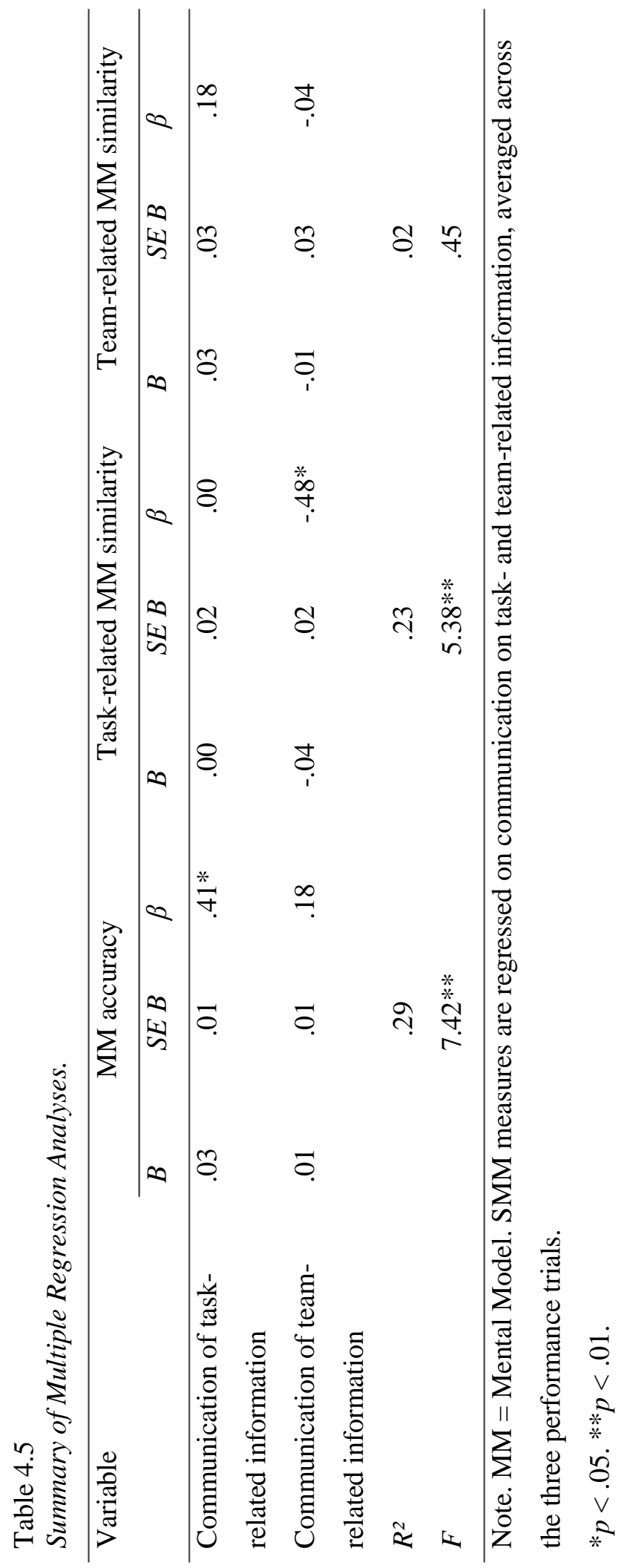




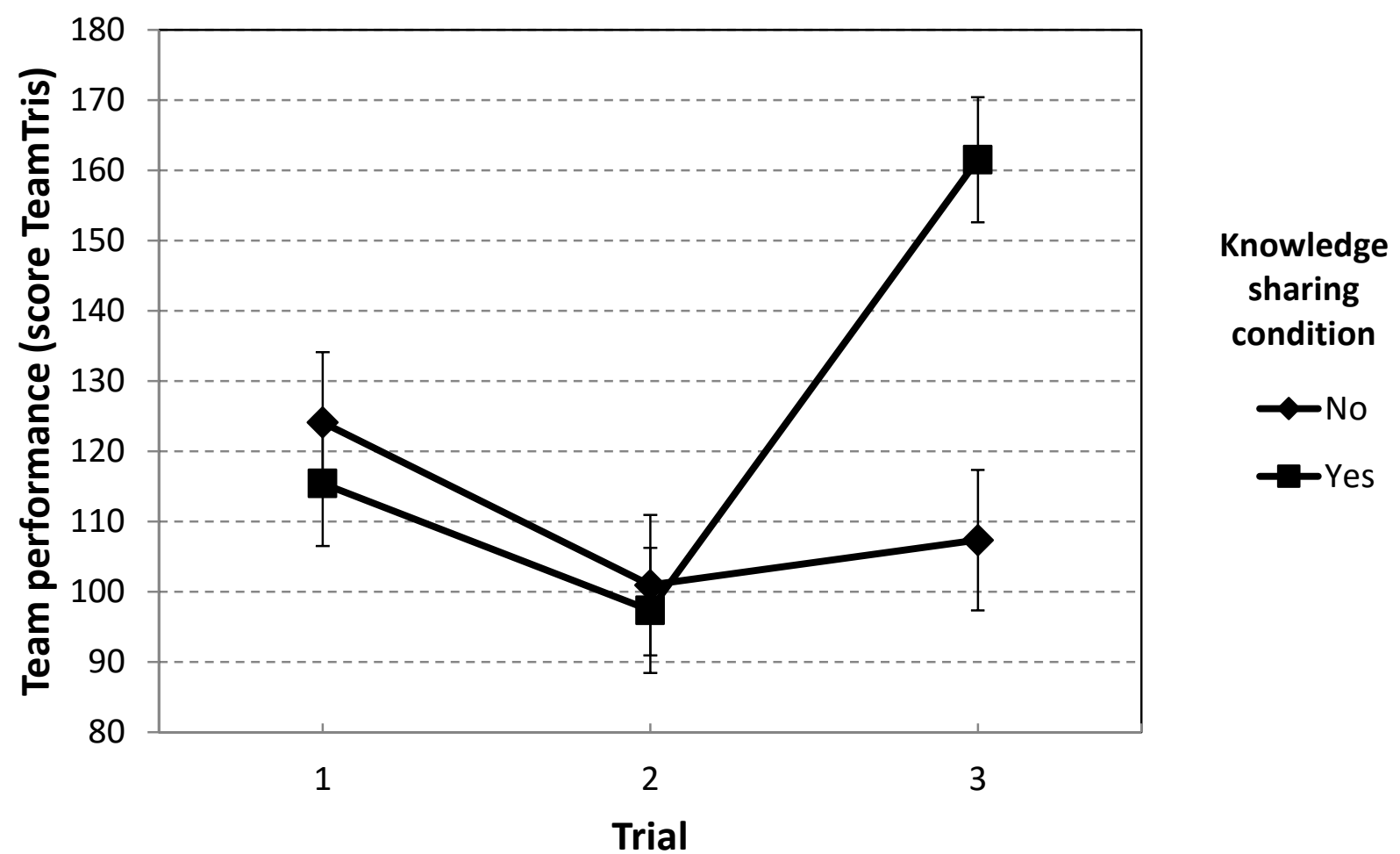

Figure 4.3. Team performance as a function of trial and condition.

\section{Discussion}

The aim of this study was to investigate the potential benefits of knowledge sharing on the development of SMMs among team members. In addition, we tested the relationship of knowledge sharing and SMMs with communication, and examined effects on team performance. This is an important investigation as it is insufficiently clear how team members can be supported in developing SMMs to reach better team outcomes. Overall, we can conclude that guiding team members in knowledge sharing on task and team aspects has positive effects on team cognition, processes and performance.

The results show that knowledge sharing improved the development of team members' SMMs, their communication during subsequent game play and their team performance during the final trial. More precisely, knowledge sharing led to more similar team-related mental models and mental models that were more accurate. Team members communicated more task- and team-related information during task performance after they have engaged in knowledge sharing on task and then team aspects. Particularly, the increase in task-related communication influenced the development of more accurate mental models between team members. Mental model accuracy in turn was positively associated with team performance. The overall effects of knowledge sharing became most strongly visible in the final game trial through a substantial performance increase of teams in the knowledge sharing condition. 
The results demonstrate that knowledge sharing is an effective process to support the development of team-related mental models and accurate mental models. We show that prevailing notions on the gradual development of SMMs (Kennedy \& McComb, 2010; McComb et al., 2010) can help to design knowledge sharing effectively and guide team members in the knowledge sharing process. In addition, through differentiating between task- and team-related SMMs and between mental model similarity and accuracy, we expand previous research that imply positive effects of knowledge sharing on SMMs (Gross \& Kluge, 2012, 2014; Sikorski et al., 2012). These outcomes increase our understanding of how SMMs develop and how we can help team members in this development. For the development of effective SMMs, team members benefit from mutually exchanging their knowledge prior to task performance. Organizations can help team members in knowledge exchange by providing opportunities, i.e. time and platforms, to understand each other's tasks and discuss how to cooperate. A lack of such organizational support may hamper the development and maintenance of SMMs (Druskat \& Pescosolido, 2002).

We suggested that knowledge sharing results in more communication of relevant SMM content and that this communication in turn further affects SMMs. We found that knowledge sharing of task aspects indeed resulted in increased communication among team members about task-related information during subsequent collaboration. Similar, knowledge sharing of team aspects resulted in more communication of team-related information. This finding corroborates the information sampling bias that predicts that teams communicate more about shared than unshared information (Stasser \& Titus, 1985; Stasser \& Titus, 1987). While research has often confirmed the information sampling bias, the results are interesting in the present context. In fact, SMM theory would predict that team members communicate less after they have engaged in knowledge sharing that helped SMM development (Cannon-Bowers et al., 1993). This is because SMMs enable implicit coordination without verbal communication (E. L. Blickensderfer et al., 2010; Langan-Fox et al., 2004). Team members in the present study, however, communicated more. This shows that SMMs need time to develop to enable implicit coordination (Mohammed et al., 2010) and that the information sampling bias is particularly strong if information is initially unshared (Mesmer-Magnus \& DeChurch, 2009). An interesting investigation for future research would therefore be to test the relationship of knowledge sharing and communication in mature teams that have already developed effective SMMs and in which most information is shared from the beginning.

In contrast to frequently made statement in the literature, we predicted a positive effect of the information sampling bias. We expected that communication about shared information further helps the development of SMMs (Burke et al., 2006; Kennedy \& McComb, 2010; Uitdewilligen et al., 2010). The results partly confirm this expectation as more communication about task-related information resulted in more accurate mental models. This indicates that team members in the knowledge sharing condition repetitively examined their knowledge available in the team. First, they examined their knowledge during knowledge sharing and then during communication. This iterative 
Chapter 4

examination positively affects the accuracy of team members' mental models (Kennedy \& McComb, 2010). This indicates again that SMMs take time to develop (Mohammed et al., 2010) and any help to support this development should consider this temporal aspect. Team members in an organization should therefore have various possibilities to exchange information and examine their knowledge, informally and formally (Bartol \& Srivastava, 2002).

We also found that communication of team-related information negatively affected the development of task-related SMMs between team members. These team members communicated more information about, for example, how specific actions influence other team members.

Apparently, their increased effort to communicate this information was at the expense of available cognitive resources to develop similar task-related mental models (cf. MacMillan et al., 2004). A more detailed look into the communication transcripts of the present study indicates that team members who frequently talked about team-related aspects were actually less focused on task execution and tended to make more errors. This finding is not an exception as previous research reported similar costs of team behaviour (Barnes et al., 2008). Specifically, team members who provided back-up behaviour often neglected their own task, but also team members who received back-up from others tended to decrease their task efforts. Future research should look at the optimal conditions for team members to communicate specific content without experience adverse effects on their team cognition. It is important to help these teams to balance their communication with effective SMMs development.

A final aim of this study was to investigate the effects on knowledge sharing and SMMs on team performance. We showed that guided knowledge sharing of task- and team-related information resulted in improved subsequent performance. This outcome supports previous studies that reported similar effects of knowledge sharing in various team environments (De Vries \& Van den Hooff, 2006; Mesmer-Magnus \& DeChurch, 2009; Wang \& Noe, 2010). However, effective knowledge sharing that distinguishes task versus team aspects is a new approach and should be of help to practitioners to instigate teamwork improvements.

The relationship between SMMs and team performance was somewhat less straightforward as only mental model accuracy correlated with team performance. This shows that studying the SMMperformance relationship remains an important investigation. There is an ongoing discussion in the literature about whether similarity or accuracy of mental models contribute more to team performance (e.g. Cooke et al., 2003; Edwards et al., 2006; Marks et al., 2000; Mathieu et al., 2000). The present findings imply that mental model accuracy is a better predictor of team performance than similarity (H. H. McIntyre \& Foti, 2013). Nevertheless, the assessment of both SMM properties remains important in future research because their role may vary for different task and team contexts. Moreover, the interaction between mental model similarity and accuracy can also be predictive in team performance (e.g. Mathieu et al., 2005). 
The findings of this study have a number of implications for teamwork and team training in versatile and dynamic environments. It is important to actively stimulate teams in knowledge sharing and make them aware that this facilitates their SMMs, which will ultimately improve team performance. Organisations should acknowledge this importance of creating and stimulating opportunities for knowledge sharing, for example in the form of regular team discussions (Bartol \& Srivastava, 2002; Druskat \& Pescosolido, 2002). Team members rarely create such opportunities themselves, as they might not directly understand the benefits. In addition, in many cases they may not have the time for such extra-task activities. However, a couple of minutes before task performance may be enough for team members to develop and maintain SMMs. Repeated knowledge sharing from the outset may help team members to agree early on task strategies and on how to interact effectively within the team. The organizations plays a crucial role in the support of knowledge sharing and in the creation for opportunities for this process.

A particular interesting situation is a task environment in which knowledge sharing is necessarily limited by for example digital communication that only allows information exchange through predefined text-messages (Stedmon et al., 2007). This is for example the case during datalink technology used by many modern action teams. The question then is whether such a dynamic task environment with restricted communication possibilities among team members will hamper the development and update of SMMs. Also, it is interesting to investigate whether knowledge sharing as designed in the present study would have similar positive effects on team cognition and performance.

\section{Limitations}

The present study contributes to unravel the intricate mechanisms underlying knowledge sharing, the development of SMMs, communication and performance within a team. The results, however, may be restricted to teams that have little experience with the other team members and with the task. While it is common that team members who work in complex environment are not necessarily familiar with each other, they are usually extensively trained in their task. Future research should replicate the findings of the present study for such expert teams.

We must note that SMMs were measured after task completion. While this is quite common in SMMs research, we specifically chose this procedure so that the communication and task performance were not altered by the actual measurement of SMMs. However, this makes it difficult to directly chart the gradual development of SMMs from task-related knowledge to knowledge on team aspects. Still, we indirectly appraised this development via communication and team performance. Further research should examine SMMs development at several moments in time, to enable detailed investigations of how SMMs relate to team processes and performance. 
Chapter 4

\section{Conclusion}

To conclude, understanding the underlying processes of SMMs development received little attention in the literature so far. The present study shows that combining ideas of knowledge sharing with recent notions on SMMs development is a fruitful approach to study this process. Knowledge sharing on first task and then team aspects improve SMMs directly, as well as indirectly by encouraging team members to communicate shared information repetitively. Task- and team-related SMMs develop at subsequent moments during task performance and team members need to repetitively examine information until individual mental models converge into SMMs (Kennedy \& McComb, 2010). Organizational support designed to facilitate SMMs should be timed accordingly to lead to higher mental model accuracy and better team performance. 


\title{
Chapter 5
}

\section{When team members communicate via datalink: The positive effects of guided off- task knowledge sharing on shared mental models, team behaviour and team performance}

\begin{abstract}
Datalink technology allows concise and time-critical information exchange among team members working in complex environments. However, this technology-mediated communication medium has potential limits, which may hamper the development of shared mental models in a team. Shared mental models are crucial for effective team performance and an aversive effect on its development should be prevented. This study tests whether guided off-task knowledge sharing can help teams to develop shared mental models when working in a datalink environment. Therefore, 35 teams were assigned to either an experimental or a control condition. Results showed that guided off-task knowledge sharing resulted in higher similarity of team-related mental models and in higher mental model accuracy among team members. Team members in the experimental condition also engaged more often in the team behaviour closed-loop communication and showed a strong performance progress. Guided off-task knowledge sharing thus helps to mitigate potential hampering effects of datalink.
\end{abstract}





\section{Introduction}

Effective teamwork is important in many organizations and requires team members to have a common understanding of the task and team. This common understanding is often referred to as shared mental models (SMMs). SMMs are defined as similar and accurate knowledge structures of team members that enable them to work together smoothly and support each other effectively (Espevik, Johnsen, \& Eid, 2011; Salas, Rosen, et al., 2007). Team members with SMMs show effective team behaviours as they provide information when needed and easily coordinate their actions (Espinosa et al., 2004). In this paper, we study how SMMs and associated team behaviours develop through knowledge sharing in fresh-starting teams working in datalink settings.

The development of SMMs in fresh-starting teams presumably occurs in phases. Team members first develop SMMs about the task and subsequently about the team (Kennedy \& McComb, 2010). For the effective development of both task and team SMMs team members must actively and explicitly exchange information early in a teams' lifecycle (Okhuysen \& Eisenhardt, 2002; Zijlstra et al., 2012). If information exchange is restricted this might hamper the development of team members' SMMs and consequently affects their team behaviour and performance (Andres, 2012).

Restricted information exchange occurs if team members communicate via datalink technology (Thompson \& Coovert, 2003). Datalink is a communication link that transmits digital data between two locations, for example an air traffic control tower and an aircraft. It allows sending and receiving large amounts of information via text-based predefined messages (Neal et al., 2011). This way of communicating leaves little space for additional information exchange other than predefined messages and may consequently hamper the development of SMMs (Andres, 2012). Since team members' SMMs positively relate to team performance (DeChurch \& Mesmer-Magnus, 2010b), it is important to reveal whether team members achieve SMMs in these technology-mediated communication settings and to help them in this process (Langan-Fox et al., 2004).

This study investigates SMM development in datalink settings and thereby contributes to the field and the literature in a number of ways. Overall, the study investigates whether we can help freshstarting teams working with datalink technology to develop SMMs. We help these teams through guided off-task knowledge sharing - the process through which individuals mutually exchange information (De Vries \& Van den Hooff, 2006; Mesmer-Magnus \& DeChurch, 2009), which we designed according to several ideas in the team literature. First, we build on the idea that SMMs develop effectively if team members exchange personally held information (Gross \& Kluge, 2014; McComb, 2007; Okhuysen \& Eisenhardt, 2002; Zijlstra et al., 2012). Accordingly, we provide 'windows of opportunities' to exchange this information through knowledge sharing. Second, in line with previous research that have shown that breaks during team discussions can improve team processes and cognition (Okhuysen \& Eisenhardt, 2002; Okhuysen \& Waller, 2002) we provide opportunities for knowledge sharing between task performance, i.e. 'off-task'. Third, we apply 


\section{Chapter 5}

theoretical ideas of the phases of SMM development, which suggest that task-related mental models develop before team-related mental models (Kennedy \& McComb, 2010). Accordingly, we guide team members in knowledge sharing in a way that they successively exchange information about the task and the team.

In this study, we examine team members' SMMs directly through questioning and indirectly via team behaviours associated with SMMs and team performance. The indirect measure allows measuring SMM development over time, whereas direct measures of SMMs indicate the final SMMs at the end of the experimental sessions. The distinctions allows further understanding of the SMM concept per se and the development of SMMs.

\section{The Shared Mental Model Concept}

SMMs in dynamic environments are important as most tasks that individuals have to perform are very demanding and thus require teamwork (Fiore et al., 2010). This means that team members need to cooperate and often have to rely on information they provide to each other (Smith-Jentsch et al., 2005). SMMs help team members to fulfil these demands and align their interpretation, explanations and expectations (Mohammed et al., 2010). This makes teams more effective in various task environments such as the Army, aviation and power plants (e.g. Lim \& Klein, 2006; Smith-Jentsch et al., 2005; Waller et al., 2004). Team effectiveness results from both the similarity and accuracy of team members' mental models (Rentsch et al., 2008; Smith-Jentsch, 2009). Mental model accuracy describes how adequate knowledge represent the real world and mental model similarity is the compatibility of knowledge between team members (Edwards et al., 2006).

SMMs in a team are also believed to comprise at least knowledge about two aspects of their environment, namely the task and the team (Smith-Jentsch et al., 2005). The task-related mental model includes knowledge about task activities, procedures and equipment, whereas the team-related mental model represents knowledge about other team members and interaction patterns (Mathieu et al., 2000; Resick et al., 2010). Team members presumably have additional types of SMMs such as the team strategy mental model (Randall et al., 2011), but following previous studies we focus on task and team-related SMMs as these are the main knowledge types required in most tasks.

\section{Shared Mental Model Development}

The development of team members' SMM is based on the convergence of individual knowledge or mental models into combined or shared knowledge structures (Cannon-Bowers et al., 1993; LanganFox et al., 2004; Mathieu et al., 2010). This process depends strongly on information exchange among team members (Kennedy \& McComb, 2010). Information exchange entails that team members share personally held information and knowledge, which would otherwise be distributed among individuals. Particularly in fresh-starting teams, information is distributed and information exchange helps to converge separate mental models of individual team members into SMMs (Cooke, Gorman, Duran, et 
al., 2007; Gross \& Kluge, 2014; Klimoski \& Mohammed, 1994; MacMillan et al., 2004; Sikorski et al., 2012; Uitdewilligen et al., 2010; Waller et al., 2004). This implies that information exchange is most effective when it happens from the start of a team's life cycle (Zijlstra et al., 2012). It can reduce uncertainties about, for example, roles and responsibilities, and allows team members to quickly focus on actual task performance (Zellmer-Bruhn et al., 2004).

While team members exchange information about various aspects of their task, it is likely that task-related SMMs develop before team-related SMMs (Kennedy \& McComb, 2010; Mathieu et al., 2005). It is easier for team members to share concrete knowledge about the task, while they need a longer time period to share abstract knowledge about the team. SMM development thus presumably occurs in stages so that different types of SMMs evolve at different points in time (Kennedy \& McComb, 2010; McComb et al., 2010). This gradual process corroborates ideas of team development. These ideas imply that team members need a basic understanding of their individual task activities before they improve task effectiveness through teamwork (Kozlowski et al., 1999).

In addition to this gradual process, the development of SMMs in a team is also iterative (Burke et al., 2006; McComb, 2007). Team members tend to repetitively exchange, augment, and reinforce information until individual knowledge converges into SMMs. This process continues until team members have gained enough shared knowledge that further information exchange becomes unnecessary (McComb, 2007). Therefore, any support for the development of SMMs in teams should consider that this support requires repetitive exposure to knowledge.

\section{Team Behaviours Associated with Shared Mental Models}

Once team members have developed SMMs, this is discernible in their behaviour (Malakis et al., 2010b; Salas, Rosen, et al., 2007). SMMs among team members often become apparent in that they allocate communication resources more effectively, provide information before it is requested and offer support when needed (Salas, Rosen, et al., 2007). For example, team members with effective SMMs often atempt to avoid misinterpretation of a received message. They ask for, or provide, confirmation of the received message, or perform a cross-check by repeating the message. Such behaviours are referred to as closed-loop communication (Espevik et al., 2011). A second type of team behaviour suggesting the existence of SMMs pertains to so-called mutual performance monitoring (Salas, Sims, \& Burke, 2005). This becomes visible when team members keep track of the activities of fellow members and may timely anticipate on the information that fellow team members need.

These team behaviours are observable components of the otherwise internal states of SMMs (McComb et al., 2010). Thus, by investigating the frequency of these team behaviours during task execution it is possible to derive information about team members' SMMs. More precisely, team members with effective SMMs should more frequently engage in close-loop communication and mutual performance monitoring. Team behaviours are not only an observable indicator of SMMs, but offer the possibility to measure SMMs more continuously over time. 


\section{Datalink and its Impact on Shared Mental Model Development}

The reason to apply datalink communication in complex team environments is because of its alleged advantages over conventional voice-based communication (Neal et al., 2011). For example in air traffic control, text based messaging via datalink is a solution to the increasingly congested radio frequencies (Lancaster \& Casali, 2008). It minimizes miscommunication that is often the result of mishearing and faulty interpretation of unclear speech due to radio frequency noise or poor pronunciation (Sharples et al., 2007). Another claimed advantage of text above voice communication is that an incoming datalink message places less cognitive demand on a team member (Lancaster \& Casali, 2008). Since datalink is set up along a protocol of short and predefined text-based messages, a team member selects messages from a limited set of options, often activated by a simple mouse or touch input. A received message remains visible so that receivers do not have to remember the message actively. This means that attending to any received message may be postponed to prevent it from interrupting ongoing communication or other activities (Metzger \& Parasuraman, 2006; Stedmon et al., 2007).

Despite these claimed benefits, datalink communication in a team setting is also characterized by information loss and reduces the interaction between team members. The actual datalink creates an additional technological layer between team members and as such, forms a personal distance (Metzger \& Parasuraman, 2006). Moreover, datalink communication typically lacks what is called party-line information (Sharples et al., 2007), which pertains to the potential valuable overheard information exchange between for example two fellow team members. In datalink communication, such valuable information exchange may not be distinct, as it is merely another text message that needs active attending to. This also implies that datalink additionally burdens visual attention in already strongly visually determined environment such as air traffic control (Metzger \& Parasuraman, 2006; Voller \& Low, 2004). A final disadvantage of these electronic text messages is that they are less rich than communication by voice, as text messages typically lack non-verbal voice information, such as intonation, tone or pitch (Driskell, Radtke, \& Salas, 2003; Sharples et al., 2007; Thompson \& Coovert, 2003; Waller et al., 2004).

Consequently, datalink communication generally reduces shared access to some verbal as well as non-verbal information. And despite that team members exchange essential information via datalink, they do not necessarily gain a full shared overview of the team goals and strategies (Keyton $\&$ Beck, 2010) and may therefore be unable to update their mental models (Sharples et al., 2007). Moreover, team members have fewer possibilities to exchange, augment, and reinforce information (Driskell et al., 2003). As these behaviours are building blocks for a SMM, team members, and especially if they participate in newly formed teams, may have more difficulties to develop or maintain SMMs in a datalink setting (Andres, 2012; Cramton, 2001; Thompson \& Coovert, 2003).

Such difficulties will shape both the cognition and performance of the entire team. For example, technology-mediated communication may prevent that team members agree on a joint 
strategy. This in turn may lead to uncoordinated task activities, confusion, and potential inefficiencies (Andres, 2012; Thompson \& Coovert, 2003). An avalanche of these inefficiencies may lead to severe mistakes in a highly complex and interdependent task context (Cramton, 2001; Driskell et al., 2003). A fitting solution is required for these potential sources of hampering and even harmful effects of datalink communication on team cognition and team performance.

\section{Facilitating Shared Mental Model Development in a Datalink Setting through Knowledge Sharing}

The present study suggests that providing team members with guided off-task opportunities for knowledge sharing facilitates the development of a team's SMMs in a datalink setting (Kennedy \& McComb, 2010). Knowledge sharing is the exchange of relevant information, knowledge and expertise (De Vries \& Van den Hooff, 2006). It comprises providing and receiving explicit and tacit knowledge through, for example, communication, observation and apprenticeship (Bartol \& Srivastava, 2002). Team members who engage in knowledge sharing create a joint understanding of previously individual knowledge (De Vries \& Van den Hooff, 2006) and consequently should develop more similar and accurate mental models (Cannon-Bowers et al., 1993).

Empirical evidence for such a positive effect remains scarce, despite the theoretical relatedness of knowledge sharing and SMMs. Until now, only a few studies investigated the relationship between knowledge sharing and SMMs (Gross \& Kluge, 2014; Sikorski et al., 2012; Ure, Dewar, Pooley, Lloyd, \& Jaegersberg, 2004; Xiang, Lub, \& Gupta, 2013). Two of these studies found first evidence that knowledge sharing improves SMMs (Gross \& Kluge, 2014; Sikorski et al., 2012). The present study contributes to this previous research as it focuses on team members working with datalink technology. This makes knowledge sharing as facilitator for SMMs specifically interesting because it may help to overcome the shortcomings of datalink technology as described earlier.

Guided off-task knowledge sharing includes team members to have planned breaks between task sessions (off-task). During these breaks, they can actively exchange information and thus have additional opportunities to synchronize their knowledge (Druskat \& Pescosolido, 2002). Breaks in task activity are effective because team members can share knowledge on task and performance issues, which they may otherwise not address (Okhuysen \& Eisenhardt, 2002). In a datalink setting it is particularly important because the restricted possibility to exchange information hampers knowledge sharing (Andres, 2012). The overall aim is to help team members exchanging distributed information and sharing knowledge that is difficult to access when they solely exchange text-based information via datalink.

In line with ideas on SMM development, guided off-task knowledge sharing should support the gradual and iterative process of mental model convergence (Cannon-Bowers et al., 1993; Kennedy \& McComb, 2010; McComb et al., 2010). More precisely, it should consist of subsequent sessions that aid the development about task-related SMMs first and then team-related SMMs. This would support 


\section{Chapter 5}

the assumed process that team members share task knowledge before they share team knowledge (Kennedy \& McComb, 2010). Guided off-task knowledge exchange should also offer the possibility of repetitive information exchange to increase the chance that individual knowledge converges into SMMs (McComb, 2007).

In this study, we guide team members in knowledge sharing during three off-task training sessions by applying principles of cross-training. Cross-training is a training form where each team member receives information regarding the task and responsibilities of other team members (Ellis \& Pearsall, 2011). It describes specific actions to support knowledge sharing and improve mutual information exchange among team members through positional clarification, team discussion and positional rotation (e.g. Cooke et al., 2003; Dalenberg, Vogelaar, \& Beersma, 2009; Gorman et al., 2010; Marks et al., 2002; Sikorski et al., 2012). The general idea is that subsequent off-task knowledge sharing sessions have individual effects on SMM development, team behaviours associated with SMMs and on team performance. This leads to four specific hypotheses outlined below.

\section{Hypotheses}

The discussed theoretical background and practical concerns of team functioning in a datalink setting lead to four hypotheses with regard to how guided off-task knowledge sharing may aid the development of SMMs. The general idea is that information exchange during subsequent sessions of guided off-task knowledge sharing positively affect team members' SMMs. This should become discernible via direct appraisal of mental model accuracy and the similarity of task- and team-related mental models after all performance sessions (Rentsch et al., 2008; Smith-Jentsch, 2009).

Hypothesis 1. Guided off-task knowledge sharing improves the development of SMMs (task/team-related mental model similarity and mental model accuracy) of teams working in a datalink communication setting.

The positive effect of guided off-task knowledge sharing on SMMs should also become discernible via team behaviours associated with SMMs (Salas, Rosen, et al., 2007). This study focuses on two types of team behaviours observable during task execution, namely closed-loop communication (Espevik et al., 2011) and mutual performance monitoring (Salas et al., 2005). The idea is that if team members develop more effective SMMs through guided off-task knowledge sharing, they should also more frequently engage in closed-loop communication and mutual performance monitoring. Both behaviours are observable during task execution and offer an indirect measure of SMM development over time (Marks et al., 2002). We hypothesize:

Hypothesis $2 a$. Guided off-task knowledge sharing increases the frequency of closed-loop communication in teams working in a datalink setting.

Hypothesis $2 b$. Guided off-task knowledge sharing increases the frequency of mutual performance monitoring in teams working in a datalink setting. 
We finally argue that the positive effect of guided off-task knowledge sharing on SMM development should become discernible in team performance. SMMs positively predict team effectiveness (DeChurch \& Mesmer-Magnus, 2010b) and a support for team members to develop more effective SMMs in a datalink setting should result in improved team performance.

Hypothesis 3. Guided off-task knowledge sharing increases performance in teams working in a datalink setting.

\section{Method}

To study the hypotheses we conducted an experiment in a datalink setting and manipulated the opportunity for guided off-task knowledge sharing. This manipulation resulted in two condition, namely and experimental condition in which team members were guided in knowledge sharing and a control condition in which team members became no additional opportunities for knowledge sharing.

\section{Participants}

105 undergraduate university students participated in the study for course credits. They were randomly assigned to 35 three-person teams. Participants' age ranged from 18 to 49 years $(M=21.12$ years, $S D$ $=3.73$ ) and 81 were women. Given the randomized study design, groups did not differ significantly on any of the control variables ( $p$ 's $>.05$ ). Participants gave informed consent prior to the study, which was approved afore by the local ethics commission.

\section{Experimental task}

Figure 5.1 shows the interface of the experimental task used in this study - a dynamic team environment called TeamTris (Sander et al., 2015). TeamTris is a PC-based, cooperative version of Tetris $^{\mathrm{TM}}$ and designed for usage in experiments. The environment is equipped with a simplified datalink communication interface and intends to simulate the cognitive requirements of dynamic team environments. While the air traffic control task inspired the design of TeamTris, it includes aspects of teamwork that occur in other complex team tasks, too. It requires both individual and team effort and allows studying team cognition and behaviour in a dynamic setting. Critical aspects that are required to play TeamTris successfully are coordination, strategizing, situation awareness, role interdependency and planning.

In TeamTris, three team members sit at separated PC's and work together by individually manipulating game pieces in a visually shared game field. Identical to regular Tetris ${ }^{\mathrm{TM}}$, the team goal is to complete lines of blocks at the bottom of the field. For this purpose, each team member performs one of three roles namely, Planner, Controller 1 and Controller 2. The Planner assigns game pieces from the upper game field, which continuously fills with 2 × 3 game pieces from a pre-defined list, to the game area of one of the controllers. The Planner can also put a game piece into holding for later assignment to a controller. The system randomly drops a new game piece if no falling piece is in the 


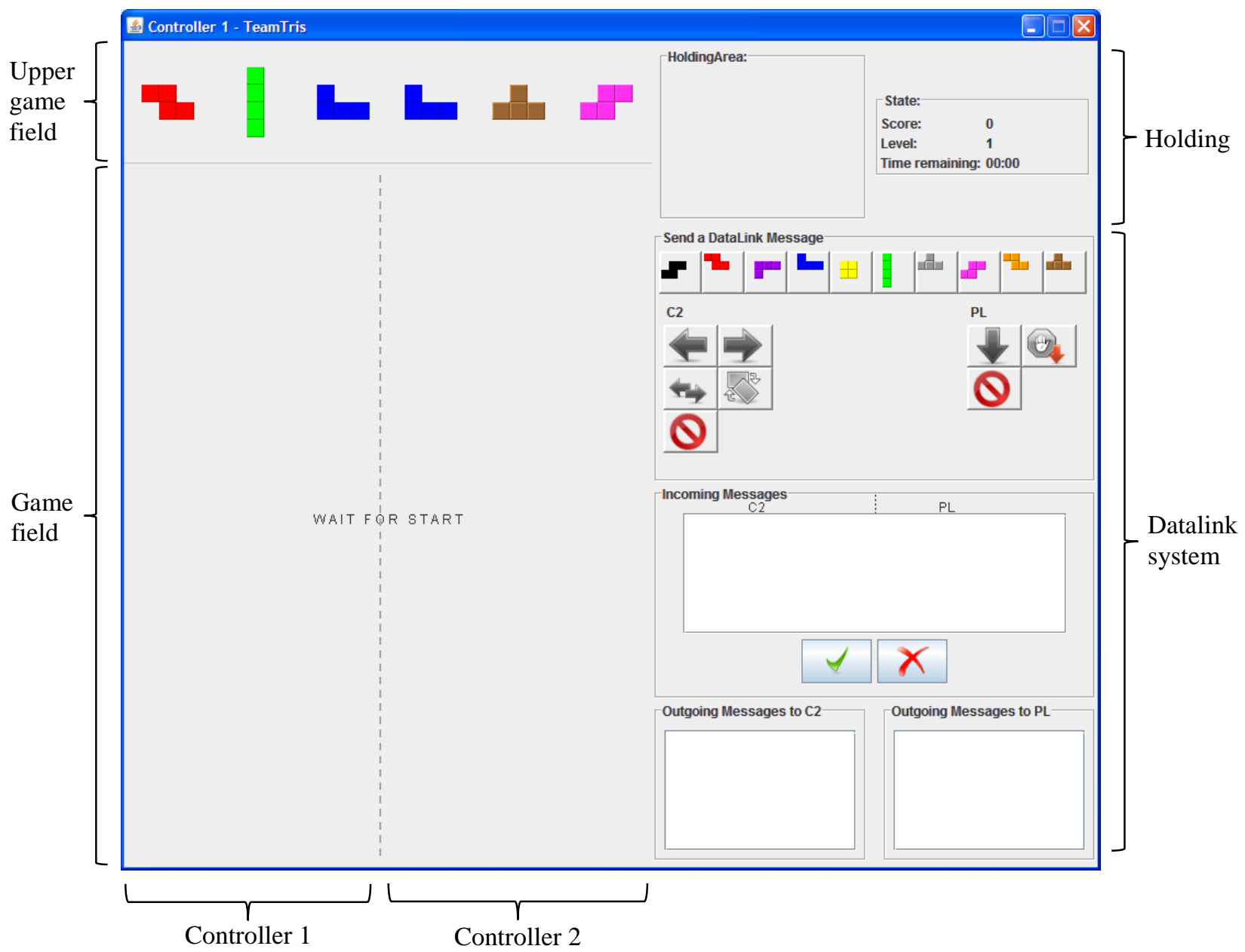

Figure 5.1. Interface of TeamTris from the perspective of Controller 1

game field and the Planner has not yet assigned the next piece. Both controllers are responsible for directing every falling game piece through a dedicated area to a suitable spot on the bottom of the game field. When the game pieces form one or more completely closed lines across the two playing areas, these lines will disappear and the team yields points. Goal of the task is to jointly clear as many lines as possible throughout the two playing areas in a given period.

The interface for datalink is located on the right side to the game field (see Figure 5.1). Datalink allows communication of concise, factual information including intended actions and requests for actions. The interface shows a number of buttons captioned with icons indicating their function, such as various types of game pieces, control buttons to manipulate the positioning of the game pieces, and buttons to indicate the receipt of a message or to send messages. A datalink message is composed by combining a number of these buttons. For example, the Planner can announce a planned I-shape for Controller 1 by clicking on the I-shape and then on the down arrow for Controller 1. This message then appears in both the outgoing message box of the Planner and the incoming message box of Controller 1. The receiver can either ignore (no action), approve (click 'check'), or reject (click 'cross') the message. If rejected, the message disappears from the controller's inbox, and 
becomes red print in the Planner's outbox, while an accepted message turns into green print. Nothing happens if the controller ignores the message.

The task is designed as a team task and several cooperative game elements have been integrated to ensure role interdependency. Four important elements are: (a) penalty points are assigned when the system instead of the Planner drops a game piece, (b) bonus points are assigned when four lines are cleared simultaneously, (c) the possibility to exchange game pieces between controllers, and (d) the restriction that some game pieces cannot be rotated by either Controller 1, Controller 2 or by both. Effective team performance in TeamTris depends on the team members' ability to develop a strategy that fits individuals' competencies and takes role interdependencies into account.

\section{Procedure}

Participants were informed that they would take part in a 2-hr team performance study. Upon arrival, they were randomly assigned to one of three team functions (Planner, Controller 1, or Controller 2) of a 3-person team. All teams were randomly assigned to either the experimental or the control group. Participants were instructed not to talk with each other during the experiment except when the experimenter specifically asked for it. After team members were seated at one of three computers, separated by dividing walls, they entered demographic information (age, gender, nationality and experience with computer games). Next, they carefully read their role-specific task description for the player role each member was assigned to. Each description contained both unique role-specific information and unique task information that was relevant for successful team performance. Therefore, most relevant information was initially unshared. A brief introduction session to familiarize with the task environment by a hands-on session of 5 minutes was followed in which no communication was required. Subsequently, participants watched an instruction video on how to communicate via the datalink interface. The video was supplemented with printed material that participants could consult during the experimental sessions. This was followed by three successive familiarisation session of 5 minutes each and with increasing complexity by including non-rotatable shapes. Next, participants accomplished three equally difficult gaming trials, each preceded by a break for guided off-task knowledge sharing or a filling task depending on the assigned group (see Figure 5.2). Exact instructions are described below. The game trials took 10 minutes each. Datalink messaging and team scores were logged by the system for each trial. After the final trial, participants completed two tests designed to query SMMs. 


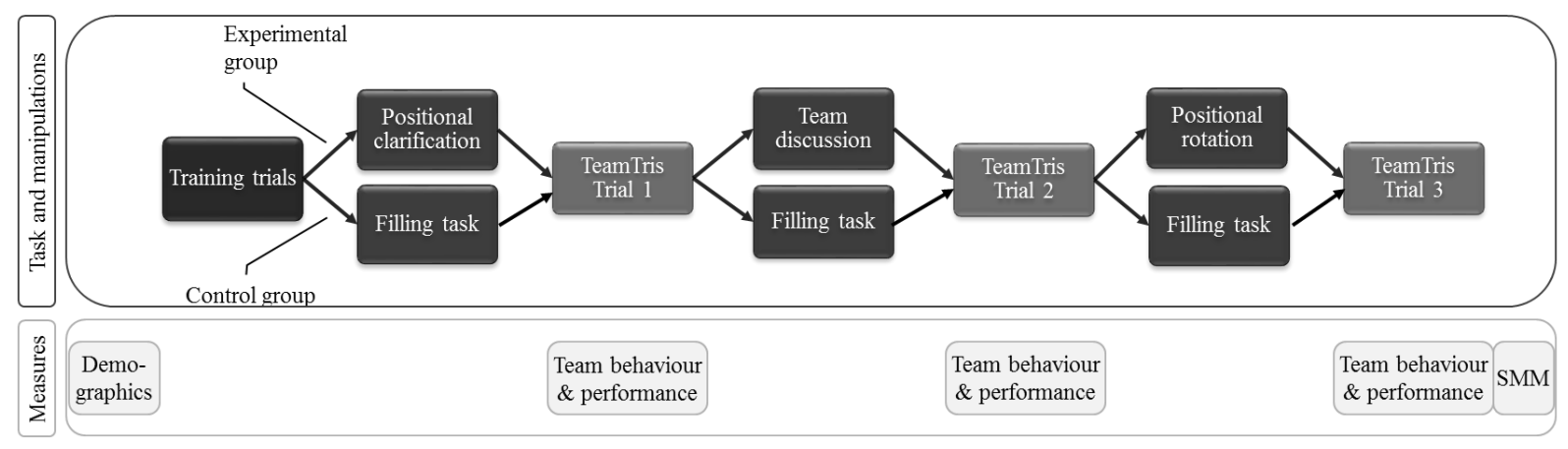

Figure 5.2. Schematic representation of the time line of an experimental session.

\section{Guided off-task knowledge sharing}

Team members in the experimental condition participated in three sessions of guided off-task knowledge sharing (see Figure 5.2). According to principles of cross-training these sessions included positional clarification, team discussion and positional rotation (E. Blickensderfer et al., 1998; Gorman et al., 2010).

Positional clarification. During positional clarification, participants in the experimental condition first reread their own task description and then reviewed the task description of the other team members. Task descriptions were presented on the pc-screens for five minutes and participants were asked to read the descriptions carefully and make sure they fully understand their own and other's tasks. The review was limited to individual reading and there was no discussion among participants. This off-task session aimed to aid information exchange between team members of initially unshared information about the task and responsibilities of the other team members in order to build a task-related SMM.

Team discussion. During the second off-task session, participants participated in a team discussion for five minutes. They sat down around a table and were instructed to discuss on their previous performance and on how they can improve performance in subsequent sessions. Participants were asked to exchange information about five items: (1) Describe each other's tasks and goals; (2) How well do you communicate as a team? Can you improve your communication? (3) Do you work together effectively? How can you improve teamwork?; (4) What is your team strategy?, (5) How can you distribute the workload among each other? The goal of this session was to actively exchange information that participants could otherwise not address during task execution and that help them to develop a team-related SMM.

Positional rotation. The third off-task session entailed a positional rotation of 2 x 4 minutes and aimed to aid both task- and team-related SMM development. In this session, participants played TeamTris for 4 minutes on each of the other positions, i.e. Controller 1, Controller 2 or Planner, respectively. Before the game started, they received a shortened task description of the new position including instructions for game controls. Positional rotation allowed participants to repeat and apply 
earlier shared knowledge and help them to integrate individual information into their SMMs. The opportunity of hands-on experience of previously shared and discussed information intended to support the iterative process of SMM development.

Control condition. Participants in the control condition performed filling tasks during the off-task sessions. During session 1, they reread their own task description for five minutes. They were asked to review the description carefully and make sure they fully understand their task. In session 2, participants summarized their task in a written format. They were instructed to give a description of their function for someone who is unfamiliar with the task. They had five minutes to complete the task description and could proceed to an unrelated IQ test if they have finished the task description in less than five minutes. In the final off-task session, participants spent an additional $2 \times 4$ minutes on playing TeamTris as before. All filling tasks intended to keep participants in the control condition occupied with TeamTris for the same amount of time as participants in the experimental condition. The main differences between the conditions were that participants in the experimental condition exchanged information of the other functions through knowledge sharing whereas participants in the control condition focused solely on their own function.

\section{Measures}

\section{Shared mental models}

Mental model similarity. The mental model similarity measure was based on an earlier used approach (Mathieu et al., 2010; Smith-Jentsch et al., 2005). To determine similarity, this approach calculates a consistency index rather than a consensus index, based on the assumption that individuals' mental models are compatible but not identical. In TeamTris, several alternative actions can be effective for solving a problem and it is important that individuals have similar perception of the prioritization of actions and not the absolute value of these actions (Mathieu et al., 2010). For example, if two team members rate a specific action as the most desirable action from a list of multiple actions this implies similar shared knowledge structures between the two members - even though they may assign another absolute value of desirability.

Task-related mental model similarity was assessed using the cue-strategy association measure (Smith-Jentsch et al., 2005). We designed four scenarios, which depicted a possible situation in TeamTris, each with four possible actions. Participants rated the desirability of executing each action on a six-point scale ranging from 1 (very undesirable) to 6 (very desirable). Task-related mental model similarity was the inter-member correlation between these ratings within each team.

Team-related mental model similarity was assessed using the positional goal interdependency measure (Smith-Jentsch et al., 2005). We developed four scenarios, which described a possible course of action of the Planner or of one of the controllers. Participants then rated how this action would affect each role on a 7-point scale ranging from 1 (strong negative influence) to 7 (strong positive 


\section{Chapter 5}

influence). Team-related mental model similarity was the inter-member correlation between these ratings within each team.

Mental model accuracy. Team members' mental model accuracy was assessed by a questionnaire consisting of six statements regarding task rules and knowledge about other's responsibilities. Participants rated whether the statement was right or wrong, or whether they did not know the answer. The approach to compute mental model accuracy of a team was comparable to the one applied by Cooke and colleagues (2003). Individuals' mental model accuracy was composed of two proportions: the proportion of correctly answered responses and 1 minus the proportion of items answered with "I don't know". The sum of these two proportions was divided by two. Equation 1 shows this computation with $\mathrm{A}(\mathrm{i})$ being the accuracy of person $\mathrm{i}, \mathrm{C}_{\mathrm{i}}$ being the number of correct answers given by person $\mathrm{i}, \mathrm{U}_{\mathrm{i}}$ being the number of unknown answers given by person $\mathrm{i}$, and $\mathrm{T}$ being the total number of possible answer.

$$
A(i)=\frac{\frac{C_{i}}{T}+\left(1-\frac{U_{i}}{T}\right)}{2}
$$

Mental model accuracy of a team was then calculated by averaging the individual mental model accuracy scores per team, yielding scores ranging from 0 (no accuracy) to 1 (complete accuracy).

\section{Team behaviours}

Closed-loop communication. Closed-loop communication was operationalized as confirmatory behaviour among team members (Espevik et al., 2011). This was determined by the sum of all rejections and approvals of datalink messages, divided by the sum of all datalink messages that were sent during one trial.

Mutual performance monitoring. Mutual performance monitoring was operationalized as offering relevant information (Salas et al., 2005). This pertains to expected events and can be communicated in TeamTris by the Planner. The Planner may for instance announce the assignment of the next game piece before a controller request that particular piece. We calculated offering relevant information via the proportion of announced game pieces by the Planner without being requested by a controller.

\section{Team performance}

Team performance was the total team score per TeamTris trial (trial 1 - 3). This score was composed of rewarded points (cleared lines of game pieces) and assigned penalty points (non-planning and collisions of game pieces) that were both automatically logged. 


\section{Analysis}

Experimental factors were experimental condition (control, experimental) and trial (1,2,3). Analyses involved one-way ANOVA (hypothesis 1) and two-way ANOVAs (hypothesis 2 - 3) with mixed design experimental condition $\mathrm{x}$ trial $(1,2,3)$. Adaptation of degrees of freedom (Greenhouse-Geisser) on the basis of significant violation of unequal variances between conditions is reported if they led to a change in p-value. P-values of paired comparisons between factor levels were Bonferonni-adjusted.

\section{Results}

Table 5.1 gives an overview of the inter-correlations of all studied variables. The table shows some interesting correlations. For instance, mental model accuracy correlates significantly with performance during trial 2 and 3 and also with closed-loop communication during trial 2. Moreover, mutual performance monitoring in the third trial correlates with task-related SMM.

\section{Shared mental models as a function of experimental condition}

To further analyse whether the various sessions of guided off-task knowledge sharing improved mental model accuracy and similarity, one-way ANOVAs were conducted. ANOVAs tested the effect of the experimental condition on the development of SMMs. Table 5.2 shows the means and standard deviations of the SMM measures (expressed as proportions ranging from 0 to 1.0 ) as a function of experimental condition. Conform hypothesis 1 , teams in the experimental condition held more similar team-related SMMs than teams in the control condition, $F(1,33)=11.39, p<.01, \eta^{2}=.26$, and developed more accurate mental models as compared to teams in the control condition, $F(1,33)=$ $43.73, p<.001, \eta^{2}=.52$. These results confirm the positive effect of guided off-task knowledge sharing on the development and final formation of similar mental models of team aspects and accurate mental models among team members working in a datalink setting. 


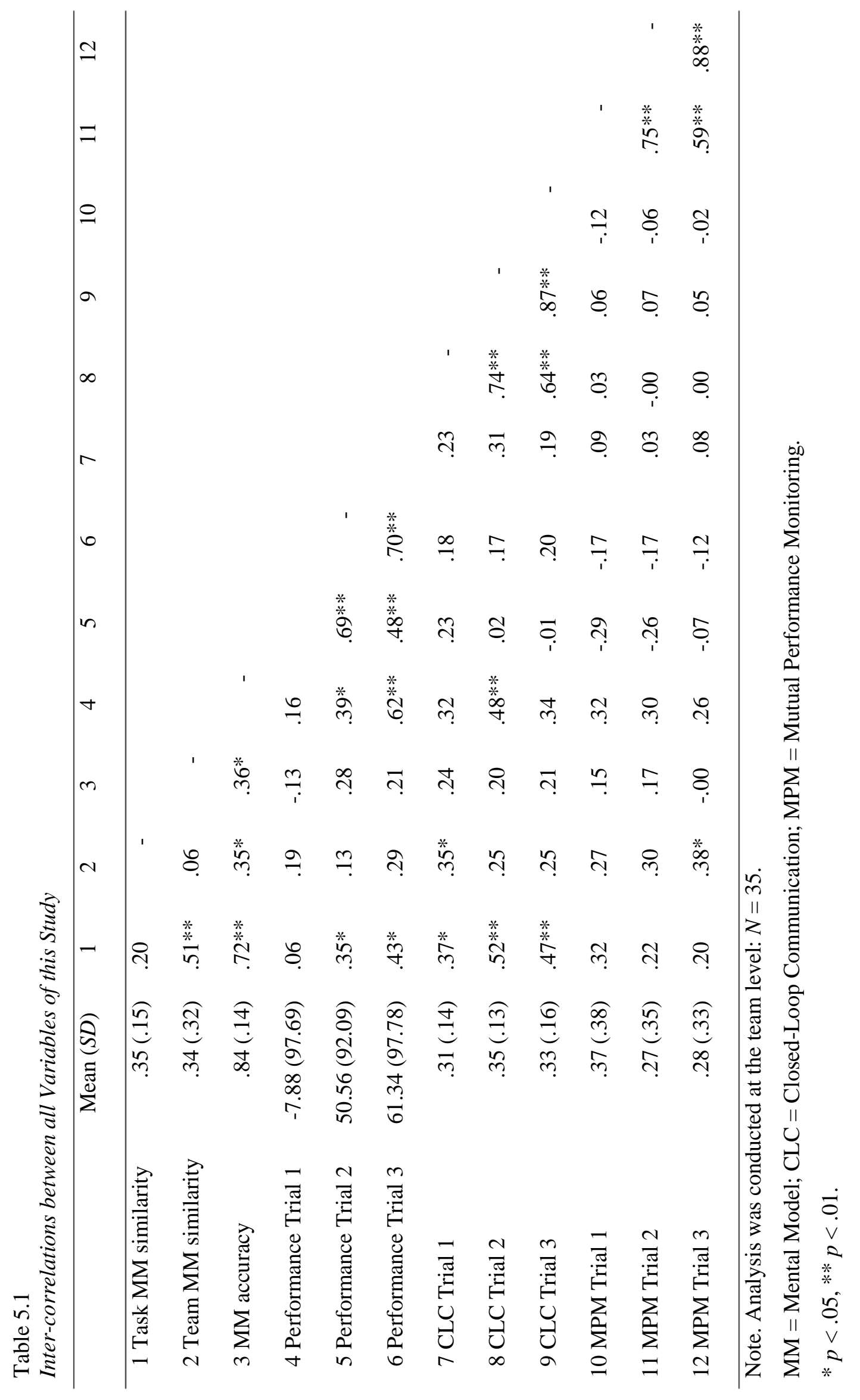


Table 5.2

Means and Standard Deviations of Shared Mental Model Measures as a Function of Condition (Control, Experimental)

\begin{tabular}{lccccc}
\hline & \multicolumn{2}{c}{ Control Condition } & & \multicolumn{2}{c}{ Experimental condition } \\
\cline { 2 - 3 } \cline { 5 - 6 } & $M$ & $S D$ & & $M$ & $S D$ \\
\hline 1. Task MM similarity & .32 & .14 & & .38 & .15 \\
2. Team MM similarity & .18 & .27 & & .49 & .28 \\
3. MM accuracy & .72 & .13 & & .94 & .06 \\
\hline
\end{tabular}

Note. $\mathrm{MM}=$ Mental Model

No differences between the two conditions were found regarding task-related mental model similarity, $F(1,33)=1.41, p=.24$. These findings suggest that those off-task knowledge sharing sessions pertaining to the task (positional clarification and partly positional rotation) had no effect on SMM development on task aspects. In the discussion section, it is argued that experience with the task environment may have sufficed to develop task-related SMMs in teams of both conditions.

\section{Team behaviour as a function of trial and experimental condition Closed-loop communication}

Table 5.3 shows the average data on proportion of confirmatory behaviour as a function of trial and experimental condition. The table suggests an increase of confirmatory behaviour in all three trials and across trials, as substantiated by statistical tests. Results show a main effect of trial $(F(2,58)=3.17, p$ $\left.<.05, \eta^{2}=.10\right)$, which was best fitted by allowing a quadratic change of trial, $F(2,58)=12.71, p<$ $.001, \eta^{2}=.31$. Paired comparison revealed significant increase from trial 1 to trial $2(p<.01)$ and a subsequent decrease from trial 2 to trial $3(p=.04)$. There was a main significant effect of experimental condition $\left(F(1,29)=9.72, p=.004, \eta^{2}=.25\right)$. Teams in the experimental condition showed more confirmatory behaviour than teams in the control condition (hypothesis 2a).

Table 5.3

Proportion of Confirmatory Communication (0 - 1.0) as a Function of Trial (1, 2, 3) and Experimental Condition (Control, Experimental).

\begin{tabular}{lccccc}
\hline \multirow{2}{*}{ Trial } & \multicolumn{2}{c}{ Control Condition } & & \multicolumn{2}{c}{ Experimental Condition } \\
\cline { 2 - 3 } \cline { 5 - 6 } & $M$ & $S D$ & & $M$ & $S D$ \\
\hline Trial 1 & .25 & .17 & & .36 & .12 \\
Trial 2 & .28 & .17 & & .42 & .12 \\
Trial 3 & .25 & .15 & & .39 & .16 \\
\hline
\end{tabular}




\section{Chapter 5}

\section{Mutual performance monitoring}

Table 5.4 shows that the Planner offered slightly more relevant information before requested by another team member in the experimental as compared to the control condition. However, this difference was not statistically significant $(p>.5)$. Hypothesis $2 \mathrm{~b}$ could therefore not be confirmed. There was a main trend of trial $F(1.43,58)=3.12, p=.07, \eta^{2}=.10$, after Greenhouse-Geisser correction for unequal variances between trials. Paired comparison revealed that all teams offered less relevant information aspects in the second trial as compared to the first trial $(p<.04)$. The discussion section will elaborate on the idea that this type of team behaviour is less relevant in TeamTris.

Table 5.4

Proportion of Offering Relevant Information (0 - 1.0) as a Function of Trial (1, 2, 3) and Condition (Control, Experimental)

\begin{tabular}{lcccccc}
\hline \multirow{2}{*}{ Trial } & \multicolumn{2}{c}{ Control } & & \multicolumn{2}{c}{ Experimental } \\
\cline { 2 - 3 } \cline { 5 - 6 } & $M$ & $S D$ & & $M$ & $S D$ \\
\hline Trial 1 & .24 & .37 & & .49 & .37 \\
Trial 2 & .18 & .33 & & .34 & .33 \\
Trial 3 & .20 & .33 & & .33 & .33 \\
\hline
\end{tabular}

\section{Team Performance as a function of trial and condition}

There was a significant main effect of trial, $F(2,58)=9.75, p<.0001, \eta^{2}=.25$. Paired-comparison between subsequent trials showed that team performance was significantly higher in trial 2 compared to trial $1(p<.0001)$ and in trial 3 compared to trial $1(p<.0001)$. Performance in trial 2 and 3 did not significantly differ and demographic variables (age, gender, nationality) did not contribute to explaining the variance in team performance. There was a main interaction between trial and condition, $F(1.8,58)=3.95, p<.03, \eta^{2}=.12$. Figure 5.3 presents this interaction and shows that team performance in trials 2 and 3 is larger for teams in the experimental condition (hypothesis 3 ).

Apparently, team performance in the experimental condition improved after the first trial but remained equal from there on. This implies that the first off-task knowledge sharing session had no immediate effect on team performance in trial 1 , while the improvement due to an off-task team discussion during the second trial was not altered further by positional rotation, which was implemented in the third off-task session prior to trial 3.

\section{Discussion}

The results of the present study demonstrate the effect of guided off-task knowledge sharing on SMMs of team members working in a datalink setting. Specifically, the results show that guided off-task knowledge sharing facilitates team members' SMMs, positively affects the frequency of closed- 


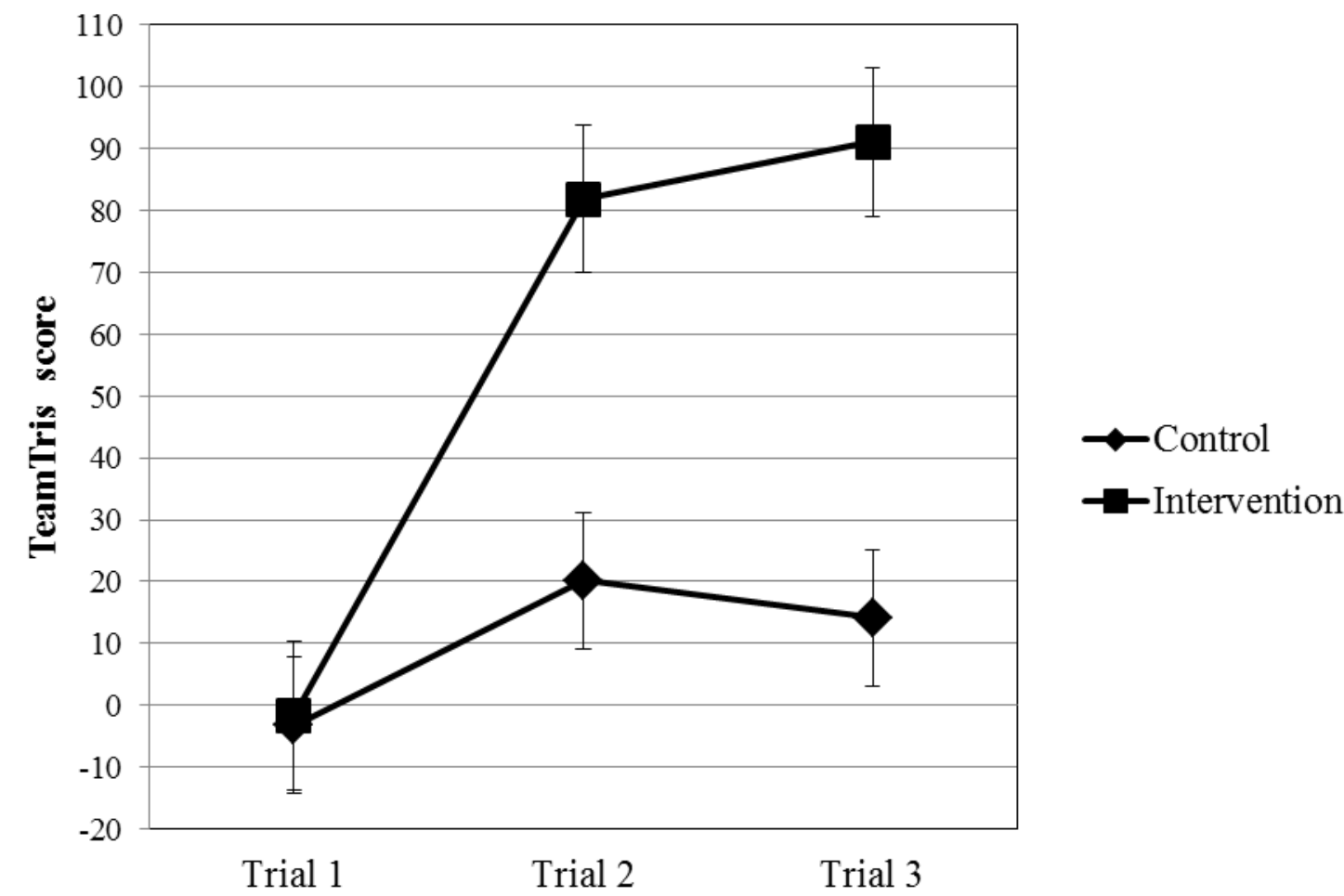

Figure 5.3. Team performance (TeamTris score) as a function of trial $(1,2,3)$ and experimental condition (control, experimental).

loop communication, and results in performance improvement over time. These findings are particularly interesting for team environments that allow for little additional information exchange among team members during task execution.

The results imply that we can not simply assume that fresh-starting teams working with datalink develop SMMs merely due to exposure to the task. Clearly, the exchange of information through knowledge sharing plays an important role in the development of SMMs (Gross \& Kluge, 2014; McComb, 2007; Zijlstra et al., 2012). In the present study, mere task exposure resulted in little team-related SMMs (18\%). Teams that exchanged additional information through knowledge sharing opportunities developed significantly more similar team-related SMMs and SMMs that were more accurate. Therefore, information exchange should pertain to more than formal messages necessary for task execution. Others already suggest that aspects of the task, such as roles and responsibilities of other team members, and aspects of the team, such as the common team strategy, is important information that should be exchanged among team members (Kennedy \& McComb, 2010).

Since datalink technology only allows the exchange of formal messages, organizations should provide team members with opportunities for knowledge sharing, i.e. mutual exchange of information. This idea corroborates with previous findings that suggest that organizational support aids the development and maintenance of SMMs in self-managing teams (Druskat \& Pescosolido, 2002). The present study shows that this finding also applies to other team and task contexts, and gives some concrete suggestions how organizational support could look like. Specifically, for teams working in 


\section{Chapter 5}

datalink settings, organizational support could take the form of guided off-task knowledge sharing. By applying principles of cross-training (Ellis \& Pearsall, 2011), organizations can take specific supportive actions to provide off-task knowledge sharing. These are positional clarification, team discussion and positional rotation (Gorman et al., 2010; Marks et al., 2002). These specific actions are in line with the idea that knowledge sharing occurs through observations, communication and apprenticeships (Bartol \& Srivastava, 2002).

Team members in this study benefited most from knowledge sharing through a team discussion. This became apparent because participants displayed more SMM-related team behaviour, namely closed-loop communication, and improved team performance in the subsequent task trial. This implies that knowledge sharing through team discussion is a fruitful method to exchange information between task trials and to aid SMM development in teams working in datalink settings. This implication expands previous research that has found that team discussions in advance of task execution positively predicts SMMs, team processes and team performance in military teams (Dalenberg et al., 2009). Similar as in the present study, team discussion was structured around a number of items and this support the idea that team members need some guidance during knowledge sharing.

The present finding also supports the idea that breaks of task execution help team members to share knowledge that they may otherwise not address (Okhuysen \& Eisenhardt, 2002). This is particularly interesting in datalink settings as this technology-mediated communication restricts the possibility to exchange (informal) information and thus hampers knowledge sharing (Andres, 2012). Overall, organizations can support team members by providing a platform and the time to discuss relevant issues of teamwork before task performance and guide them in this discussion.

It is possible that the positive effects on team processes and team performance resulted from an interaction of positional clarification and team discussion. According to notions of gradual SMM development (Kennedy \& McComb, 2010), positional clarification attempted to help task-related SMMs and team discussion aided team-related SMMs. The integration of task-related information into a SMM could have been effective not until team members exchanged team-related information. Such interactive effects between task- and team-related SMMs have been found before (Mathieu et al., 2010). However, task-related SMMs of team members in the experimental condition did not significantly differ from those of team members in the control condition. Possibly, guided-off task knowledge sharing via positional clarification was not crucial for the development of task-related SMMs in the present task context. For example, team members had a shared game display, which showed actions of other team members and might have contributed to a shared understanding of roles and responsibilities. This explanation is supported by the outcome that all teams developed relatively similar mental models of task aspects (38\%). Positional clarification may thus not have offered extra information relevant for a task-related SMM. A similar study in a different task context could give more insight into this reasoning. 
The present study revealed some unexpected findings that further research should investigate. First, closed-loop communication significantly decreased in the last trial. This may imply that team members found it no longer useful or even associated this team behaviour with additional costs (Barnes et al., 2008). Closed-loop communication, which was operationalized as confirmatory behaviour, required attention on the datalink interface instead of on the game field. It may thus have forced team members to neglect other task activities. This corresponds with the well-known drawback of datalink in domains such as air traffic control, namely that it additionally burdens visual attention of individuals (Metzger \& Parasuraman, 2006; Voller \& Low, 2004). It is particularly important to design technological interfaces in a way that support task and team performance without distracting the user form the actual task (Langan-Fox et al., 2009).

A second, related finding was that guided off-task knowledge sharing did not influence mutual performance monitoring, although it positively predicted SMMs. This does not correspond with the notion that the development of SMMs is reflected in team members' mutual understanding of information needs (Salas, Rosen, et al., 2007). Ellis \& Pearsall (2011) reported results similar to ours in low demand situations. They found that positional rotation led to mental model accuracy, but was not related to the exchange of information. It is possible that the task environment of the present study was not demanding enough to make offering relevant information via datalink essential. Again, offering information via datalink required additional visual attention and might therefore have been minimized by team members (Metzger \& Parasuraman, 2006; Voller \& Low, 2004). In other words, the visual burden may have prevented the Planner to offer information or restricted providing information to only very highly relevant messages.

The last unexpected finding was that team performance did not increase further in the last task trial after team members engaged in knowledge sharing through positional rotation. Positional rotation was designed to aid the iterative development of both task- and team-related SMMs and we assumed that this becomes discernible via better team performance. The relationship between SMMs and team performance have repeatedly been proofed (DeChurch \& Mesmer-Magnus, 2010b). Therefore, it is likely that positional rotation had no apparent additional value above knowledge sharing through team discussion. We may have offered positional rotation too early during the development of the freshstarting teams of this study. It is possible that team members were not ready to integrate the experience during the positional rotation session into their knowledge structure. Perhaps team members first need to automate their own skills before they can integrate this type of knowledge.

This study suggest a number of implications for future research and applications. The different outcomes for specific SMM indices, such as similarity versus accuracy, and task- versus team-related SMM, emphasize the importance of our choice to investigate SMM properties and types in a single study. A full understanding is only possible if these SMM indices are compared across a variety of team and task contexts (cf. Mohammed et al., 2010). Further research should reveal whether these conclusions can be generalised from laboratory teams to teams in the field. Recent studies have made 


\section{Chapter 5}

an attempt to study SMMs in the field and showed the distinctive effects of different SMM indices on team processes and performance (e.g. Mathieu et al., 2010).

The different SMM indices were only measured once after the last performance trial. This is not uncommon as dynamic shared knowledge measurements are often viewed as problematic and are typically lacking in the literature (Mohammed et al., 2010; Uitdewilligen et al., 2010). The present study attempted to avert this general shortcoming by assessing team behaviour associated within SMMs, namely closed-loop communication and performance monitoring (Salas, Rosen, et al., 2007). This indirect measure of SMMs allows measuring SMMs over time in addition to a final SMM measure at the end of the performance trials. However, there is some indications that the team behaviours studied may not necessarily be beneficial in datalink settings as they have additional costs. Consequently, the presence of SMMs may become discernible in another way than in closed-loop communication or mutual performance monitoring. Further research should reveal which behaviours reflect SMMs between team members working in datalink settings.

In conclusion, datalink and other electronic communication aids have been accepted as future applications in professions where teams are physically distributed and need to exchange big amounts of information (Andres, 2012; McComb et al., 2010; Thompson \& Coovert, 2003). These new technologies allegedly reduce communication errors and allow permanent information access. However, the intuitive benefits are in conflict with the apparent costs of datalink technology that may affect the development of SMMs and eventually team performance. Therefore, it is important to offer teams opportunities to cooperate and interact in these technology driven communication environments. This study underlines the need to support these teams by providing additional and off-task opportunities for knowledge sharing. These opportunities appear to facilitate closed-loop communication, but also improve the development of SMMs and team performance. Specifically, knowledge sharing through team discussion helps team members. It provides team members with more potential to perform well within complex man-machine systems. 


\section{Chapter 6}

\section{Team adaptation to an unforeseen system failure: Limits of the potential aids of shared knowledge and standardized communication}

The present study investigates the potential benefits of a team's shared knowledge and standardized communication in adapting to an unforeseen change by combining literature on adaptation and team performance. Each of 20 teams performed a dynamic team task and was suddenly confronted with a simulated partial system breakdown. Results show that a methodological framework designed to describe performance adaptation to an unforeseen change in individuals can also be used to model performance adaptation in teams. The system failure was followed by a performance drop and a subsequent period of gradual performance recovery. Accuracy of teams' shared knowledge correlated positively with performance before and after the change, confirming and extending the literature on shared mental models (SMMs). However, the amount of knowledge similarity did not aid teams in adapting to the unforeseen system breakdown. In addition, improving teams' standardized communication had no damping effect on the sudden performance drop and neither helped them during the subsequent recovery period. These results show that even though shared knowledge and efficient communication are of high value to team performance and success, these characteristics are limited in aiding adaptive team performance after unforeseen unique changes that force team members to update their strategies.

This chapter is based on: Sander, P. C., van Doorn, R. R. A., van der Pal, J., \& Zijlstra, F. R. H. (2015). Team adaptation to an unforeseen system failure: Limits of the potential aids of shared knowledge and standardized communication. European Journal of Work and Organizational Psychology. doi:10.1080/1359432X.2015.1006199. 



\section{Introduction}

The main issue in this study is how teams that differ in both shared knowledge and communication strategies adapt to unforeseen and unique changes in their task environment. An investigation of this issue is important as teams are often viewed as effective units to face changing and dynamic situations within organizations (Resick et al., 2010; Summers, Humphrey, \& Ferris, 2012). Teams are considered as essential for key decisions in varied settings such as hospital operating rooms, military units, sales production departments or air traffic control (ATC). These teams frequently operate in dynamic environments and have to be prepared to recognize, handle and adapt to changes in their work environment (e.g. Baard, Rench, \& Kozlowski, 2014; Burke et al., 2006; Kontogiannis \& Malakis, 2013). To adapt to these critical changes, team members are believed to apply shared knowledge, mutual understanding, and efficient and often standardized communication (Kozlowski et al., 1999).

In all, it has been recognized that effective expert teams are able to handle sudden but frequent and therefore anticipated changes (Baard et al., 2014; Burke et al., 2006; Kozlowski et al., 1999). However, it is unknown whether this also applies to unforeseen and often unique changes. These latter changes (hereafter termed unforeseen changes) refer to substantial and often unique alterations in the task environment, such as partial or total system failures or breakdowns (Weick, 1985) that a team has no experience with. These dramatic changes in the task environment are often feared by many organizations (Bainbridge, 1983), and little knowledge exists on how teams adapt to such a situation and whether they can be aided in an effective way. The questions in the present study pertain to how team performance is affected by such low frequent and unique system failures, and whether shared knowledge among team members and standardized communication help a team to adapt to these unforeseen changes.

Research on this topic is essential as an unsuccessful response to these events may have negative or even fatal consequences in a variety of work environments (Marks et al., 2002; cf. Stachowski, Kaplan, \& Waller, 2009). The present study will approach this issue by combining the literature on adaptation to changes of individuals (Lang \& Bliese, 2009) and teams (Summers et al., 2012) with existing knowledge on team functioning in terms of shared knowledge (Burke et al., 2006; Cannon-Bowers et al., 1993; Uitdewilligen et al., 2010) and communication (Burke et al., 2006; Rosen et al., 2011; Stachowski et al., 2009). More specifically, whether a team experiences a sudden performance drop followed by a recovery phase when the team members face an unforeseen change in the task environment in the form of a unique partial system breakdown is tested. Such a performance pattern after an unforeseen change can be assumed on the basis of two notions, namely coordination flux (Summers et al., 2012) and phases of adaptation in individuals (Lang \& Bliese, 2009) as it will be explained in the next section.

The present study further tests recent theory (Burke et al., 2006; Rosen et al., 2011) by investigating whether shared knowledge and standardized communication within a team helps the 


\section{Chapter 6}

team members to adapt more effectively to an unforeseen and unique change in the task environment. These aspects should aid adaptive team performance as team members should have a common understanding of the nature of the change and can communicate effectively about how to adapt to it (e.g. Randall et al., 2011)

\section{Modelling Adaptive Team Performance: Distinguishing Adaptation Phases}

It is important to note that the observation of potential effects of an unforeseen change on team performance is only possible when the team is monitored over time (Ackerman, 1992; Burke et al., 2006; Murphy, 1989; Waller, 1999). An unforeseen unique change in the task environment at one point in time may have sudden but also subsequent gradual effects on team performance (e.g. Chan, 2000; Kozlowski et al., 1999; LePine, 2005; Randall et al., 2011). These temporary and temporal performance changes will be captured in the present study by applying a methodological framework, namely discontinuous growth modelling, which has been successfully used to study adaptive performance in individuals over time (Lang \& Bliese, 2009).

Discontinuous growth modelling allows specifically testing adaptation to an unforeseen change in the task environment during different phases of performance. These phases are an initial skill acquisition (SA) followed by two consecutive phases of adaptation. During initial SA a team becomes familiar with a new task and should gradually improve in performance (Ackerman, 1992). A subsequent unforeseen change in the task environment then requires the use of specific strategies that may not have been part of the team's repertoire prior to that change (Lang \& Bliese, 2009). This implies that such a change typically creates two phases within a team (Burke et al., 2006; Randall et al., 2011; Rosen et al., 2011). The first phase is characterized by a so-called coordination flux (Summers et al., 2012), which reflects a period of uncoordinated behaviour and confusion within the team that makes it difficult to reapply learned skills. During the second phase, team members manage to gradually overcome this period by subsequently developing new interaction patterns (Summers et al., 2012) and by establishing new behavioural or cognitive goal-directed actions (Burke et al., 2006; Rosen et al., 2011).

This study will first test whether these successive alterations in a team will become visible via a specific performance pattern over time. The unforeseen change should result into a visible initial performance drop referred to as transition adaptation (TA; Lang \& Bliese, 2009) since learned behaviours and strategies fail in the new situation. This signifies the temporary coordination flux (Summers et al., 2012). While team members continue to perform the task, effective teams should develop new strategies and adjust their behaviour. This subsequent reacquisition adaptation (RA) becomes discernible via a gradual increase in performance and thus characterizes a second period of skills acquisition to adapt to the new situation (Burke et al., 2006; Rosen et al., 2011).

As discontinuous growth models have only been applied to individual performance adaptation (Lang \& Bliese, 2009), it is the first aim of the present study to add to the literature by testing whether 
the two successive adaptation phases are also discernible in adaptive team performance after an unforeseen change. Indeed performance tends to decrease temporarily in teams that experience a member change during task performance because established coordination patterns become inappropriate (Summers et al., 2012). We would expect a similar detrimental effect on performance after an unforeseen system breakdown because this requires re-evaluation of both coordination and task strategies. We specifically test whether teams perform worse immediately after the change compared to before the change (TA), and whether performance scores slowly increase again during subsequent task execution (RA). This leads to the first hypothesis:

Hypothesis 1: Team adaptation to an unforeseen change becomes visible in an initial drop in team performance (TA) followed by a performance increase (RA).

\section{Relationship between Adaptive Team Performance and Shared Knowledge}

Discontinuous growth modelling also allows testing differences in characteristics of teams during each phase of adaptation. It therefore provides the methodological basis to investigate whether team adaptation to an unforeseen change is aided by shared knowledge among team members. Shared knowledge, which is also often referred to shared mental models (SMMs), pertains to knowledge structures of team members that help them to interpret and explain the task and each other's behaviour in a similar and accurate manner (Cannon-Bowers \& Salas, 2001). The potential aid of SMMs in adaptive team performance makes sense as they are generally viewed as essential for effective team performance (Burke et al., 2006; Cannon-Bowers et al., 1993; Uitdewilligen et al., 2010). Studies show that shared knowledge that pertains to the task environment, but also to a team's functioning and interaction, positively affect team effectiveness (DeChurch \& Mesmer-Magnus, 2010b; Mathieu et al., 2005). These shared knowledge structures, that in the present study pertain to task and team aspects, thus allow team members to have a similar understanding of the task and make it possible for team members to coordinate their actions accordingly (Marks et al., 2001; Mohammed et al., 2010).

To study the potential help of SMMs among team members to team adaptation to an unforeseen change, it is important to distinguish between two apparent aspects of shared knowledge structures, namely similarity and accuracy. Mental model similarity refers to how compatible or consistent team members' knowledge is (Marks et al., 2000), whereas mental model accuracy reflects the degree to which this knowledge represents reality (Edwards et al., 2006). This distinction is important as team members, for instance, may have a shared view about how to coordinate their work, yet being wrong about the efficiency of this coordination (Lim \& Klein, 2006). Moreover, prior research showed that mental model similarity positively relates to team members' perception of their effectiveness, whereas accuracy is strongly related to actual team performance (Resick et al., 2010). Results about the role of both aspects with team performance remain inconsistent (Mohammed et al., 2010), which emphasizes the need to include both aspects in the present adaptation study. 
When team members face a change in the task environment, mental model accuracy should help them to understand the nature of the change adequately and find effective strategies to handle the new situation (Randall et al., 2011). They have an accurate understanding of the consequences of these strategies and are able to choose and apply the best strategy for the specific situation. The degree of mental model accuracy may therefore eventually enable team members to adapt to unforeseen changes irrespective of the degree of mental model similarity.

Knowledge similarity further benefits adaptive performance as team members may quickly agree on one strategy and coordinate their actions more easily. It thus enables rapid strategy selection (Rico et al., 2008) and should minimize the coordination flux in unforeseen situations (Summers et al., 2012). In addition, team members who have developed similar mental models in the past may have available more mental resources to assess a new task environment (Hockey, 1997; Kahneman, 1973; Rouse \& Morris, 1986). This benefits their ability to efficiently adjust their shared knowledge and adapt to the new situation (LePine, 2005).

These ideas about knowledge accuracy and similarity corroborate the conceptual model of team adaptation (Burke et al., 2006; Rosen et al., 2011) that envisions SMMs as input and outcome of the adaptive cycle in response to a significant change in the environment. Burke et al. (2006) propose that shared knowledge should aid the assessment of the environment for salient cues, facilitate the formulation and execution of a plan, and eventually help the process of team adaptation. In fact, they suggest that proactive adaptation is impossible in the absence of SMMs. Recent research has found first empirical evidence for these theoretical assumptions, as team members with similar and accurate mental models about strategic priorities and implications adjust existing strategies more effectively (Randall et al., 2011).

The present study tests this aspect of Burke et al.'s model of adaptation (Burke et al., 2006; Rosen et al., 2011) in terms of TA and RA (Lang \& Bliese, 2009) after an unforeseen partial system failure. Accordingly, it is expected that the performance drop after the change is less severe for teams with similar and accurate mental models and is followed by a faster recovery of task performance. This leads to the following two hypotheses:

Hypothesis 2. Mental model similarity negatively relates to the size of the initial performance drop after an unforeseen change and positively relates to the increase in performance after the unforeseen change.

Hypothesis 3. Mental model accuracy negatively relates to the size of the initial performance drop after an unforeseen change and positively relates to the increase in performance after the unforeseen change. 


\section{Effects of Standardized Communication on Team Adaptation}

This study additionally tests whether standardized and systematic communication aids a team in adapting to an unforeseen and unique system failure. It is long known that successful teams communicate effectively and exchange information in a clear and accurate manner by using standardized terminology (Espevik et al., 2011; Kanki, Folk, \& Irwin, 1991; Kring, 2005; Xin \& Zhiming, 2005). This is especially important in procedure-driven and dynamic environments. Examples are ATC and firefighting (Cannon-Bowers \& Salas, 1998; Keyton \& Beck, 2010; Uitdewilligen et al., 2013). In fact, the failure to communicate in a structured way in these environments is a frequent cause of accidents (Malakis et al., 2010b).

Of course, the effectiveness of established interaction patterns invariably decreases when the task context and/or the team settings are altered (Salas, Rosen, Held, \& Weissmuller, 2009; Stachowski et al., 2009; Wilson et al., 2007). Team members then need to quickly re-adjust their communication behaviour to the new situation to sustain a sufficient level of team performance. In the present study, half of the teams are instructed to practice standardized communication prior to and to apply it during the experimental session. The main question is whether this instruction will help these teams after the unforeseen change in the task environment (Burke et al., 2006; Stachowski et al., 2009; Uitdewilligen et al., 2013).

One perspective on the role of standardized communication patterns in team adaptation asserts that successful team behaviour, including the use of standard terminology, does not aid adaptation after an unforeseen change (Gersick, 1988). This idea is based on assertions that existing communication patterns may become obsolete after the change (Stachowski et al., 2009). Indeed, an unforeseen change creates a coordination flux of unstructured interaction patterns in all teams (Summers et al., 2012). This may however be different in teams that practiced in advance to use standardized communication protocols, which are still (partly) beneficial after the unforeseen change.

Such an idea supports recent notions on team adaptation that assumes that communication plays a central role in adaptive team performance (Burke et al., 2006; Rosen et al., 2011; Stachowski et al., 2009). Accordingly, communication is an individual-level process that aids team members carrying out a new and adaptive plan, which consequently helps them to adapt effectively. Information should thereby be articulated in a clear and accurate manner and with proper terminology.

These ideas have been successfully applied in real teams, for example to instruct aircraft crews in using communication protocols and as additional help in retaining information in memory, to aid team members in handling new situations (Salas, Wilson, \& Edens, 2009). In addition, members in teams with stable interaction patterns behave more predictably, which in turn should facilitate team adaptation to unforeseen changes (Kanki et al., 1991; LePine, 2005). The idea is that stable and especially standardized communication will be beneficial after the unforeseen change in providing a firm basis for a team to update knowledge and adapt skills to the new tasks. In other words, providing 

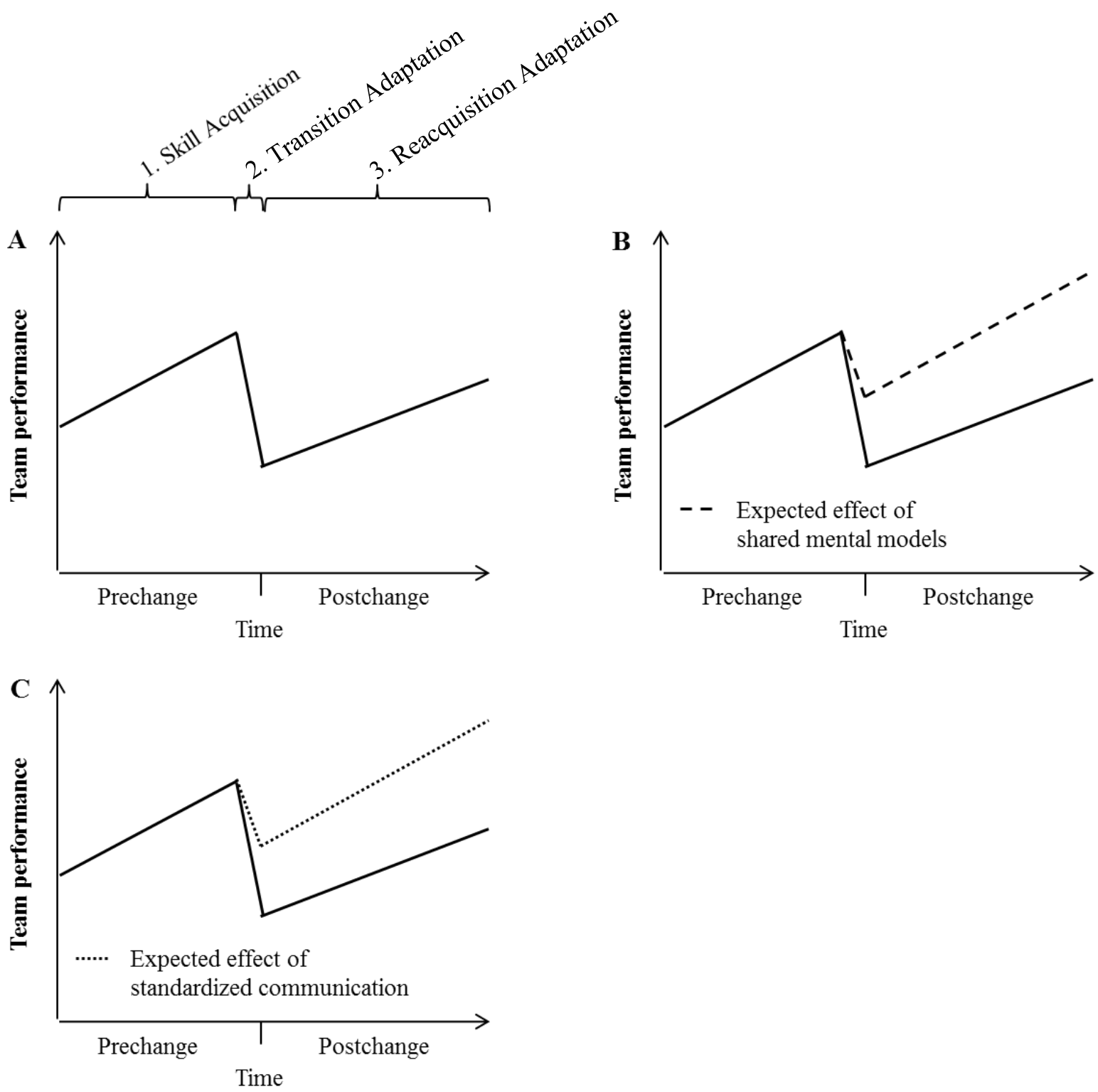

Figure 6.1. Hypothesized team performance patterns and the effects of SMMs and standardized communication: (A) mean performance pattern before and after the change (hypothesis 1), (B) effects of SMMs on team performance (hypotheses 2 and 3), and (C) effects of standardized communication on team performance (hypothesis 4).

the means to a team to apply standardized communication prior to an unforeseen change should be beneficial after the change (Burke et al., 2006; Rosen et al., 2011). This leads to the final hypothesis:

Hypothesis 4. A team that uses standardized communication before an unforeseen change will display a smaller drop in performance after the unforeseen change followed by a faster increase in team performance.

In sum, this study on team adaptation to unforeseen changes tests four hypotheses based on contemporary notions of adaptive performance in individuals (Lang \& Bliese, 2009) and in teams (Baard et al., 2014; Burke et al., 2006; Rosen et al., 2011; Uitdewilligen et al., 2013). Figure 6.1 provides an overview of the experimental design including the hypothesized pattern of team 
performance before and after the unforeseen change, and the effects of shared knowledge and communication on adaptive team performance. Team performance is studied along three phases and is expected to follow a specific pattern (hypothesis 1). In the first phase teams become acquainted to the new task environment resulting in a marked team performance improvement over time, referred to as the initial acquisition phase.

It is investigated whether adaptive team performance after an unforeseen change can be in essence described by two subsequent phases, namely TA and RA. The establishment of the two phases in the literature on individual adaptation gives reason to hypothesize that a similar performance pattern is discernible in teams. The second and third hypotheses test whether shared knowledge within a team may be beneficial in team adaptation. We distinguish between similarity and accuracy of shared knowledge and expect that both mental model similarity (hypothesis 2) and accuracy (hypothesis 3) support adaptive team performance. The final hypothesis tests whether there are potential benefits of standardized communication to adaptive team performance (hypothesis 4).

\section{Method}

\section{Participants and team assignment}

Sixty undergraduate psychology students (42 females) participated in an experiment for course credits or gift vouchers. Their average age was 20.37 years $(S D=2.03)$. Participants were randomly assigned to a team resulting in 20 teams with three members each. Every participant was assigned to one of three team roles within a team. Participants gave informed consent prior to the study, which was approved by the local ethics commission.

\section{Team task}

Teams worked on a task within a dynamic environment provided by TeamTris (Van der Pal \& Justen, 2011). TeamTris is an adaptation of the well-known Tetris ${ }^{\mathrm{TM}}$ game that allows team members to cooperate with each other. Team-critical aspects built in this task include the need to communicate, develop shared situational awareness, understand role interdependencies among team members, plan ahead, and develop adequate team strategies.

In TeamTris, three team members work together, namely one planner and two controllers. Team members are each seated behind individual personal computers, connected via a local network, and are able to communicate via headsets. Their team goal is the same as in regular Tetris ${ }^{\mathrm{TM}}$, namely to complete lines of blocks at the bottom of the screen. These blocks consist of various geometrical shapes, called game pieces, and are assigned by the planner to each controller. The controllers can then move a game piece until these reach the screen bottom or other game pieces that has descended before them. Positioning of a game piece by a controller is possible via rotation and via horizontal movement within the boundaries of a controller's area. If the planner fails to assign a game piece timely, that is, 


\section{Chapter 6}

before the present game piece in a controller's area stopped its downward movement, the system automatically assigns a piece to this controller. Planners have the option to place one piece in a temporary hold if they prefer to assign one or more other pieces first. Identical to players in regular Tetris ${ }^{\mathrm{TM}}$, successful controllers connect falling pieces into horizontal lines of blocks at the bottom of the screen. Full block lines that stretch across the two controller areas disappear and yield points. The game pieces still present in both the controller areas lower by the number of lines that disappear. The team's goal is to clear as many lines as possible.

The shared task requires team members to work together closely as their roles and responsibilities are mutually dependent. The four most important of these dependencies are: (a) penalty points are assigned when the planner fails to timely select a piece so that the system selects one, (b) bonus points are assigned when four block lines (across the two areas) are cleared simultaneously, (c) controllers have the option to strategically move a game piece to the area of the other controller, and (d) some pieces cannot be rotated by either controller 1, controller 2, or by both. These game pieces are colour coded. For example, controller 1 cannot rotate orange T-shaped pieces but are able to rotate a T-shape with another colour.

\section{Implementing the unforeseen change: A simulated partial system failure}

To simulate a partial system failure, the configuration of TeamTris changed in two ways as shown in Figure 6.2. The general team goal remained the same, but the changes required rethinking and made it necessary to apply new strategies.

First, after the simulated system failure both the controllers could no longer see the areas of both their team mates. The planner kept view of all areas. Second, the colour codes of all game pieces were altered. For example, the L-shape was violet in the prechange configuration and green in the postchange configuration. Despite these drastic changes, team members not necessarily noticed all new aspects immediately. Since the task interface of the planner remained intact, the controllers first needed to inform the planner about this change before the planner could fully understand the new situation. In addition, the new colour codes of the game pieces were particularly relevant for the dysfunctional game pieces. As these only infrequently appeared, an assessment of the situation was required first. Both unforeseen changes were designed to introduce uncoordinated behaviour and confusion, that is, coordination flux. The changes made it more difficult for all team members to use recently learned task strategies and communication habits. The question was whether a team's accurately shared knowledge and standardized communication would support team adaptation and thus lead to a smaller performance drop immediately after the change and a subsequent performance recovery. The specifics of how standardized communication was implemented in this study will be detailed in the next section. 


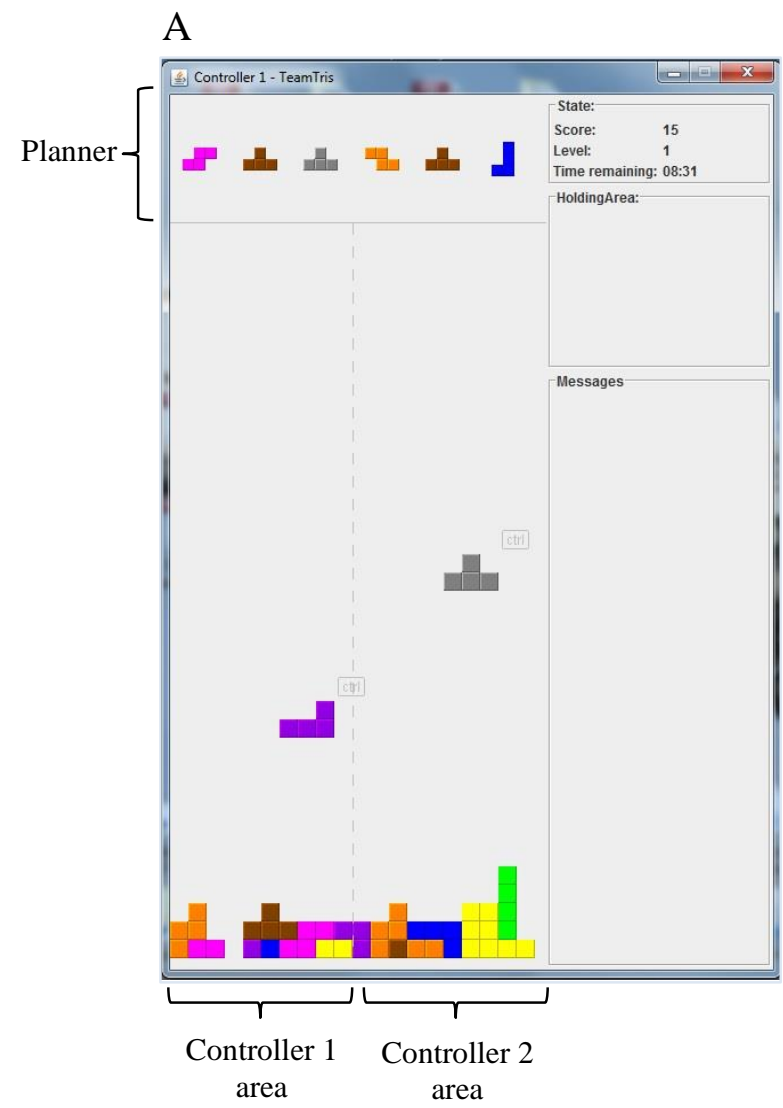

B

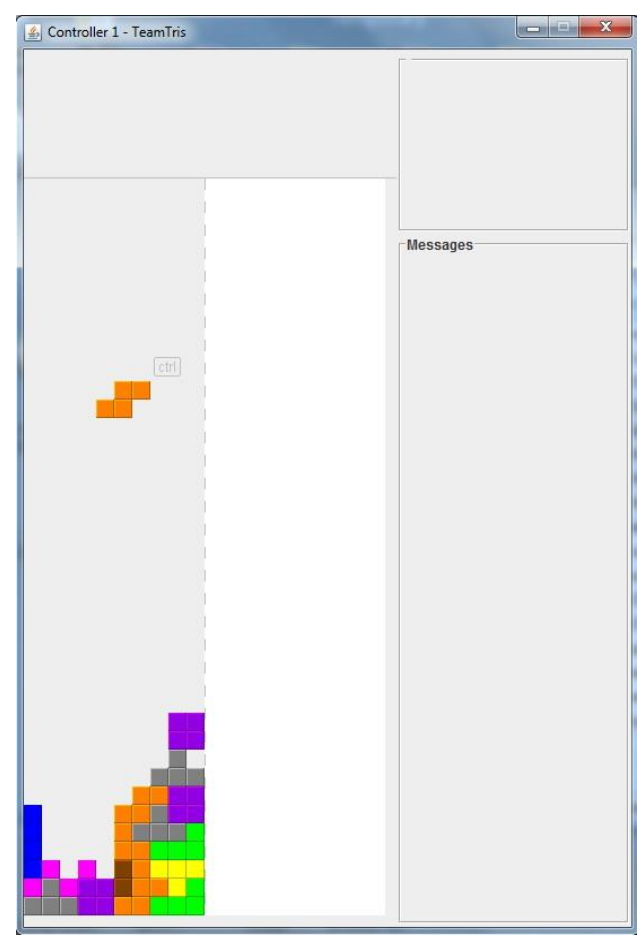

Figure 6.2. TeamTris interface for controller 2 before (A) and after the unforeseen change (B). Panel $\mathrm{B}$ is seen from the perspective of controller 2 who is unable to see the areas of the planner and controller 1. Similarly, controller 1 was unable to perceive the areas of the teammates, while the planner kept full vision. In situation B, colour coding was altered for all game pieces.

\section{Standardized communication}

Half of the teams in this study were assigned to the standardized communication condition and the other half to the control condition. The purpose of the standardized communication manipulation was to create an effective communication protocol to aid team adaptation to the unforeseen partial system breakdown. In the standardized communication condition, communication protocols were practiced at the beginning of the experimental session. More specifically, practice aimed to teach team members to use standardized terminology for game pieces and standardized communication protocols for the instructions they needed to give each other during task performance. For that purpose, team members were instructed to identify game pieces and name them by their shape instead of their colour. In addition, team members learned to communicate concisely by giving short, standardized instructions to each other. Standardized communication process comprised of three elements: a receiver, an instruction, and a game piece name. For example, in a situation in which controller 1 would ask the planner to assign an O-shape, controller 1 would say: "Planner give O." All team members read a training handbook that consisted of three parts. It first explained the terminology of the game pieces according to their geometrical shape. The second part described the instructions protocol, and the final 


\section{Chapter 6}

part provided several examples to illustrate the standardized communication flow among team members.

Manipulation Check. To test the effectiveness of the standardized communication training, participants in the standardized communication condition replied to 20 multiple-choice questions. The first 10 questions assessed whether participants could adequately identify a piece based on the terminology and shape recognition. For example: "Which of the following blocks is called an L?" The last 10 questions assessed whether participants had learned to use the trained communication protocols. For example: "If two controllers want to change blocks, which of the following instructions is correct?" We considered the training as effective as teams had an average score of $75 \%(S D=5.55)$ correct answers and $60 \%$ functioned as the minimal criterion.

\section{Procedure}

Upon arrival, participants gave informed consent and completed a demographic questionnaire.

Participants were seated behind a personal computer and read a detailed description of their assigned role. Each participant also read the task description of the other roles to ensure that all team members were informed about the general set-up of TeamTris and the different responsibilities within the game. After the instructions, teams in the control condition started the experimental session, whereas teams in the standardized communication condition first received 10 min of communication training and the subsequent training test, prior to the experimental session.

All teams played six trials of TeamTris, each lasting $10 \mathrm{~min}$. A pilot study revealed that three trials sufficed for a team to become familiar with the game and to improve in performance, which is why three pre- and three postchange trials were chosen. During the first three trials team members played the game in the prechange configuration. The final three trials were played in the changed configuration in which game piece colours were different, and controllers could only view their own area of responsibility. After each trial, team members had a 2 min break to discuss their team performance and task strategy. At the end of the sixth TeamTris trial, SMMs were assessed.

\section{Measures}

Team performance. The final score of each TeamTris trial (Trial $1-6)$ represented team performance, which resulted in six team performance measurements. Each score was composed of reward points (cleared lines) minus the assigned penalty points (nonplanning and collisions of game pieces). A team's final performance score would be negative when the penalty exceeded the reward. Note that we distinguished these scores from combined performance scores before the change (Trial 1 -3) and after the change (Trials 4 - 6) for a more comprehensive presentation of descriptive statistics.

Mental model similarity. Mental model similarity was assessed by applying a scenariobased approach that has been successfully used in dynamic team environments (Mathieu et al., 2010; Smith-Jentsch et al., 2005). The assumption is that SMMs are compatible and not identical and 
therefore should be represented by a consistency rather than a consensus index. For TeamTris (and in dynamic environments as ATC) it is important that team members have a similar perception of the prioritization of possible actions and without necessarily knowing the absolute value of these actions (Mathieu et al., 2010).

Task-Related Similarity. We used the cue-strategy association measure to assess taskrelated mental model similarity (Smith-Jentsch et al., 2005). Four scenarios represented a possible situation in TeamTris each with four possible actions. Participants rated the desirability of executing each action on a 6-point scale ranging from 1 (very undesirable) to 6 (very desirable). The intermember correlation between these ratings within each team represented task-related mental model similarity.

Team-Related Similarity. To assess team-related mental model similarity we applied the positional goal interdependency measure (Smith-Jentsch et al., 2005). We again developed four scenarios, which now each described a possible course of action of one of the team members. Participants then rated the impact of each action on the other roles on a 7-point scale ranging from 1 (strong negative influence) to 7 (strong positive influence). The intermember correlation between these ratings within each team represented team-related mental model similarity.

Mental model accuracy. To assess team members' knowledge accuracy, participants filled in a questionnaire consisting of six statements that participants rated with right, wrong, or unknown. A statement regarded task-related and teamwork-related aspects at the same time since this knowledge elements were closely interwoven in the task applied. For example, one statement was "A controller can simultaneously control several blocks in his game field." The accurate answer requires both an understanding of the task rules and knowledge about other team members' responsibilities. We therefore calculated a general accuracy index rather than team- or task-specific mental model accuracy indices. The calculation was based on an approach used by Cooke and others (2003). Individuals' mental model accuracy was composed of two proportions: the proportion of correctly answered responses and 1 minus the proportion of items rated as unknown. The sum of these two proportions was divided by 2 . Equation (1) illustrates this computation with $\mathrm{A}(\mathrm{i})$ being the accuracy of person $\mathrm{i}, \mathrm{C}_{\mathrm{i}}$ is the number of correct answers given by person $\mathrm{i}, \mathrm{U}_{\mathrm{i}}$ is the number of unknown answers given by person $\mathrm{i}$, and $\mathrm{T}$ is the total number of possible answer.

$$
A(i)=\frac{\frac{C_{i}}{T}+\left(1-\frac{U_{i}}{T}\right)}{2}
$$

Mental model accuracy of a team was then computed by averaging the individual mental model accuracy scores per team, yielding scores ranging from 0 (no accuracy) to 1 (complete accuracy). 


\section{Chapter 6}

\section{Statistical analysis}

The data were analysed with the nlme package (Pinheiro, Bates, DebRoy, \& Sarkar, 2011) of the open source software R (R Development Core Team, 2011) via the restricted maximum likelihood (REML) estimation. Multilevel analysis tested discontinuous growth models describing an initial performance growth, a sudden drop and a reacquisition of performance. For that purpose three time-based variables representing the initial performance growth and the two subsequent adaptive performance phases were created (Level 1) and nested within team-level variables (Level 2), pertaining to SMM measures and standardized communication condition. Details about these variables are provided in the next sections. The analysis involved two steps. First, performance changes over time (across six trials at Level 1) were assessed. Subsequently, SMM variables and standardized communication (at Level 2) were added as interaction terms and to function as potential team explanations of the performance changes over time.

\section{Results}

\section{Descriptive statistics, intercorrelations and intraclass correlation}

Table 6.1 summarizes the descriptive statistics and intercorrelations of the study variables. Results indicate that only mental model accuracy significantly correlated with performance both before and after the change. In addition, team- and task-related mental model similarity intercorrelated positively. Standardized communication was not related to team performance and to the SMM indices.

Table 6.1

Means, Standard Deviations, and Intercorrelations of Study Variables

\begin{tabular}{|c|c|c|c|c|c|c|c|c|}
\hline Variable & $\mathrm{M}$ & SD & 1 & 2 & 3 & 4 & 5 & 6 \\
\hline 1 Prechange performance & 266.50 & 405.61 & - & & & & & \\
\hline 2 Postchange performance & 383.15 & 359.72 & $.66^{* *}$ & - & & & & \\
\hline 3 Task-related MM similarity & .52 & .16 & .26 & .24 & - & & & \\
\hline 4 Team-related MM similarity & .36 & .23 & .16 & .26 & $.47 *$ & - & & \\
\hline $5 \mathrm{MM}$ accuracy & 8.92 & 1.48 & $.80 * *$ & $.74 * *$ & .08 & .15 & - & \\
\hline 6 Standardized communication & .50 & .51 & .31 & .18 & .14 & -.16 & .29 & - \\
\hline
\end{tabular}

$* p<.05$ (two-tailed), $* * p<.01$ (two-tailed)

$N=20$. Pre-change performance $=$ Trials $1-3$; Post-change performance $=$ Trials $4-6 ; \mathrm{MM}=$ mental model; standardized communication was coded as follows: $0=$ control, $1=$ standardized communication.

The intra-class correlation coefficient type I (ICC1; Bliese, 2000) for performance appeared to be .45 . This value represents the proportion of variation in team performance explained by differences between teams across the six task trials. This substantial variance among teams justified further analysis of team performance changes over time. 


\section{Level 1 analysis: Describing team performance changes over time}

The analysis that allowed team performance to vary across time was meant to test the first hypothesis, namely whether team adaptation would become discernible after an initial SA by a sudden drop in performance after the unforeseen change, and a subsequent RA during the trials after the change. These subsequent phases were captured and tested by three change variables: SA - a linear increase of performance; TA - a main difference before and after the change; and RA coded as a linear increase in performance after the change. The coding schemes of SA, TA, and RA are presented in Table 6.2 (Lang \& Bliese, 2009).

Table 6.2

Coding of Change Variables in the Discontinuous Growth Model

\begin{tabular}{|c|c|c|c|c|c|c|c|}
\hline \multirow[b]{2}{*}{ Variables } & \multicolumn{4}{|c|}{ TeamTris session } & \multirow[b]{2}{*}{5} & \multirow[b]{2}{*}{6} & \multirow[b]{2}{*}{ Interpretation } \\
\hline & 1 & 2 & 3 & 4 & & & \\
\hline \multicolumn{8}{|c|}{ Coding of change variables } \\
\hline SA & 0 & 1 & 2 & 3 & 4 & 5 & Linear performance increase across all six sessions \\
\hline TA & 0 & 0 & 0 & 1 & 1 & 1 & Drop in performance immediately after the change \\
\hline RA & 0 & 0 & 0 & 0 & 1 & 2 & $\begin{array}{l}\text { Linear performance increase after the change relative to } \\
\text { performance before the change }\end{array}$ \\
\hline SA2 & 0 & 1 & 2 & 2 & 2 & 2 & Linear performance increase before the change \\
\hline $\mathrm{SA} 2^{2}$ & 0 & 1 & 4 & 4 & 4 & 4 & Quadratic increase before change \\
\hline $\mathrm{RA}^{2}$ & 0 & 0 & 0 & 0 & 1 & 4 & Quadratic increase after change \\
\hline
\end{tabular}

$\mathrm{SA}=$ skill acquisition; $\mathrm{TA}=$ transition acquisition; $\mathrm{RA}=$ reacquisition adaptation.

The results of this analysis are presented in Table 6.3. They show a significant increase in the initial SA, a significant negative effect of TA, and a less strongly significant negative effect of RA during the postchange phase. The outcomes confirm the first hypothesis and indicate that the two phases of adaptation, which have been established for individual adaptive performance, also apply to adaptive team performance. The predicted values of the model as depicted in Figure 6.3 show that team performance initially improved, then suddenly dropped after the change, and slowly increased again after the change.

Testing for curvilinear changes in time. Generally, learning curves of complex skills often show nonlinear changes as they tend to rise more steeply in the beginning but reach an asymptote during the learning process (e.g., Oprins et al., 2006). To test for these potential non-linear changes in our data, we also modelled curvilinear performance changes by adding a second time variable for quadratic SA ( $\left.\mathrm{SA}^{2}\right)$ and a quadratic term for RA ( $\mathrm{RA}^{2}$; see Table 6.2). The results in Table 6.3 indicate that adding $\mathrm{SA}^{2}$ and $\mathrm{RA}^{2}$ had no main effects on performance and did not improve the model fit. Therefore, we continued the analysis with the simple linear model only. 
Chapter 6

Table 6.3

Discontinuous Mixed-Effects Growth Level 1 Models Predicting Change in Team Performance

\begin{tabular}{|c|c|c|c|c|c|c|}
\hline \multirow[b]{2}{*}{ Variables } & \multicolumn{3}{|c|}{ Model 1 (linear model) } & \multicolumn{3}{|c|}{ Model 2 (curvilinear model) } \\
\hline & Coef. & Coef. $S E$ & $t$ & Coef. & Coef. $S E$ & $t$ \\
\hline \multicolumn{7}{|c|}{ DV: Team Performance } \\
\hline \multicolumn{7}{|l|}{ Fixes effects } \\
\hline Intercept & -6.37 & 29.95 & $-0.21^{\mathrm{a}}$ & -8.45 & 33.10 & $-.26^{\mathrm{b}}$ \\
\hline SA & 95.20 & 14.55 & $6.55^{\mathrm{a}^{* *}}$ & 107.70 & 51.19 & $1.88^{\mathrm{b}^{*}}$ \\
\hline TA & -174.02 & 40.15 & $-4.33^{a^{* *}}$ & -178.20 & 61.43 & $-2.90^{\mathrm{b} * *}$ \\
\hline RA & -72.70 & 22.84 & $-3.18^{a^{* *}}$ & -122.60 & 80.88 & $-1.52^{\mathrm{b}}$ \\
\hline Quadratic SA2² & & & & -6.25 & 27.47 & $-0.23^{\mathrm{b}}$ \\
\hline Quadratic RA ${ }^{2}$ & & & & 18.70 & 27.47 & $.68^{\mathrm{b}}$ \\
\hline
\end{tabular}

$N=20 . k=60 . \mathrm{SA}=$ skill acquisition; $\mathrm{TA}=$ transition adaptation; $\mathrm{RA}=$ reacquisition adaptation

${ }^{\mathrm{a}} d f=97 ;{ }^{\mathrm{b}} d f=95$

${ }^{*} p<.05$ (two-tailed). ${ }^{* *} p<.01$ (two-tailed).

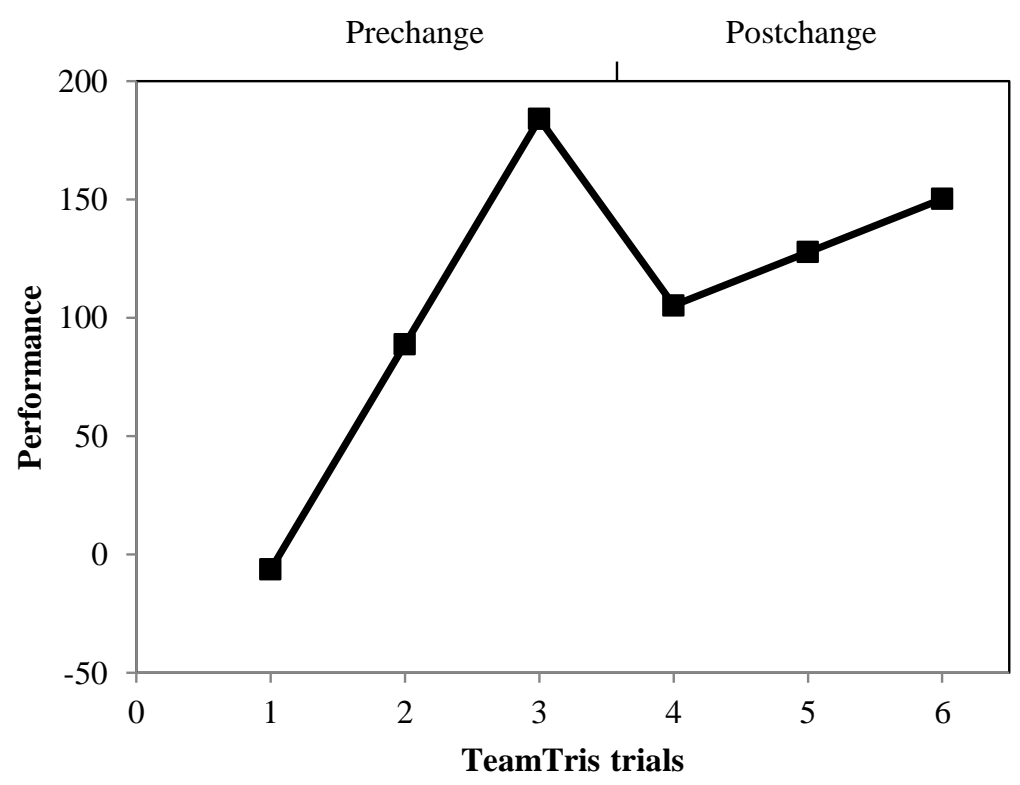

Figure 6.3. Team performance as a function of time, before and after the unforeseen change (partial system failure).

Testing for team differences. It was subsequently tested whether changes in performance were better explained when allowing variation of performance across teams and per adaptation phase. We contrasted this model with the previous one via log-likelihood ratio tests (Bliese \& Ployhart, 2002; Lang \& Bliese, 2009), which was performed per change adaptation phase. However, log-likelihood ratio tests for the simple linear models did not reveal significant results for SA: $\chi^{2} \operatorname{diff}(2)=2.13, p>$ .05 ; TA: $\chi^{2} \operatorname{diff}(2)=4.92, p=.09$; and RA: $\chi^{2} \operatorname{diff}(2)=3.32, p=.10$. These results show that the fit of 
each model does not improve by allowing the performance to vary across teams to account for potential team differences.

To verify potential impacts of shared knowledge and standardized communication on team performance over time we added Level 2 predictors as interaction terms to our current model. These cross-level interactions are permitted without significant intercept variation (group differences) when the expectations have sufficient theoretical backup (LaHuis \& Ferguson, 2009; Lang \& Bliese, 2009; Snijders \& Bosker, 1999). Level 2 predictors pertain to group characteristics that were measured once and are independent of time. We were interested whether these team characteristics would interact with the time variable to explain the change of performance over time. The specific purposes of this Level 2 analysis were first to examine the potential effect of team members' shared knowledge on team performance after the change and, second, to detect potential effects of standardized communication in adaptive team performance.

\section{Level 2 analysis: Explaining team performance via cross-level interactions}

We built four separate discontinuous mixed-effect models to test cross-level interactions to explain performance differences over time among teams. Each model tested the linear effects of each of the three subsequent phases, added by one of the four team-level (Level 2) variables, (a) task-related mental model similarity, (b) team-related mental model similarity, (c) mental model accuracy, or (d) standardized communication. The results of these models are shown in Table 6.4.

These results show that mental model similarity pertaining to both task and team did not relate to team performance and had no further influence in adaptive team performance. This implies as shown in Figure 6.4 that teams perform equally well throughout the six TeamTris trials irrespective of the degree of the similarity of team members' task (Figure 6.4A) and team (Figure 6.4B) mental models. Results further reveal that mental model accuracy leads to a general higher team performance and teams with more accurate shared knowledge also perform relatively higher after the unforeseen change (Figure 6.4C). This means that these teams have an initial performance advantage and show a relatively smaller drop in performance after the change. However, the cross-level interaction of accuracy with each single time variable, representing each of the three change adaptation phases, was not significant. In addition, the standardized communication manipulation was not related to team performance or to team adaptation after the unforeseen change (Figure 6.4D). Higher-order interactions that combined SMMs and standardized communication with each time variable were neither significant. The discussion section will elaborate on the potential implications of these results for further study and possible applications. 


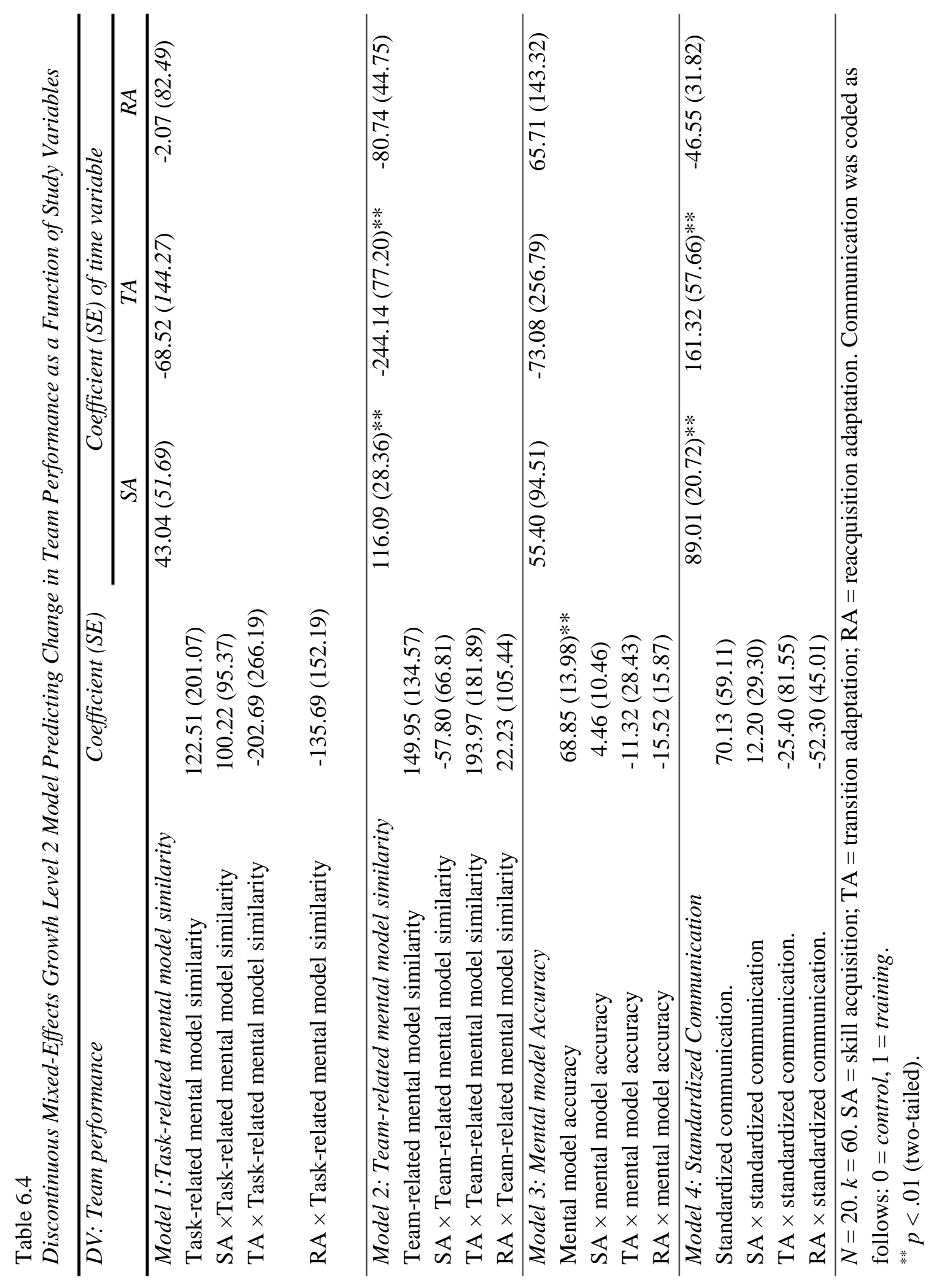


A

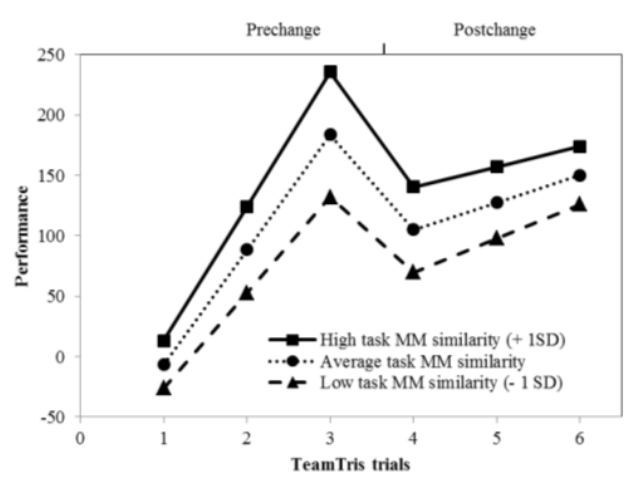

C

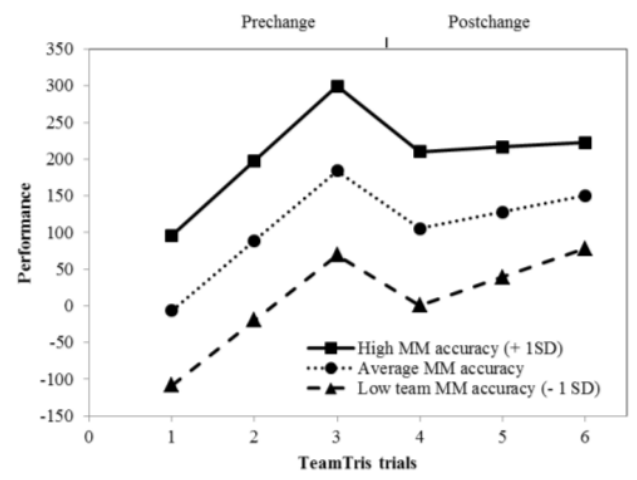

B

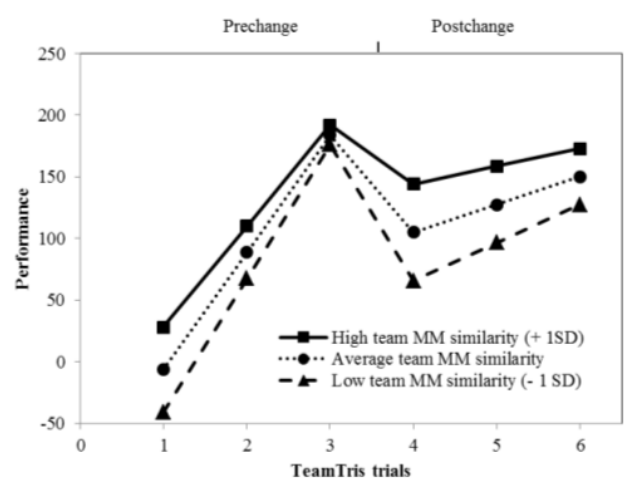

D

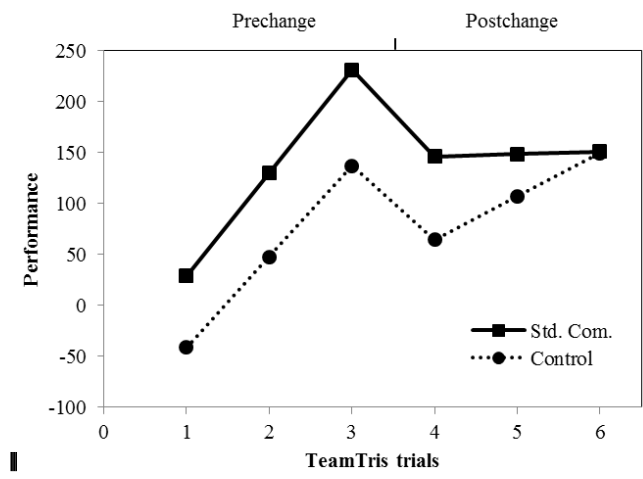

Figure 6.4. Team performance as a function of time (horizontal axes) and task-related mental model similarity (Panel A), team-related mental model similarity (Panel B), mental model accuracy (Panel C), and standardized communication (Panel D). Std. Com. = standardized communication; $\mathrm{MM}=$ mental model.

\section{Discussion}

The present study aimed to shed more light on the temporal nature of adaptive team performance and the potential benefits of shared knowledge and standardized communication. For that purpose 20 teams first performed a collaborative task in a dynamic task environment and were subsequently confronted with an unforeseen change that was implemented as a partial system failure. The results contribute to a better understanding of adaptive team performance. We could confirm that subsequent phases of adaptation observed in individuals (Lang \& Bliese, 2009) also apply to teams. Furthermore, this study affirms that mental model accuracy within a team improved performance in all phases of adaptive team performance and that team performance remains relatively high after an unforeseen change in the task environment. Accuracy of shared knowledge, however, did not specifically affect specific phases of adaptive team performance. Furthermore, the present results did not support the assumptions that mental model similarity and standardized communication protocols affect adaptive team performance after an unforeseen unique change in the task environment. These results and their implications are discussed in more detail. 


\section{Chapter 6}

\section{Theoretical implication}

Team adaptation to an unforeseen change can be described by two consecutive phases, namely a sudden performance drop (transition phase) followed by a reacquisition phase discernible by a steady increase in performance. The phases following an unforeseen change have previously been found in individuals (Lang \& Bliese, 2009) but provide new insight in team research. Although it has been shown that team members experience a sudden performance drop after a team member change (Summers et al., 2012), the present study is unique in that team members have to deal with a partial system breakdown. In addition, the study adds value to prevailing literature by showing the usefulness of discontinuous growth modelling in a team context. This methodological approach helps to better understand the temporal mechanisms of adaptive performance pattern.

The approach is also useful to test influential factors of team performance during distinctive adaptive phases. This is important because the impact of a specific factor may differ depending on the adaptive phase under investigation (Lang \& Bliese, 2009). In the present study the mechanisms and processes pertaining to standardized communication and two properties of shared knowledge were investigated during both phases to test aspects of contemporary models of team adaptation (Burke et al., 2006; Kozlowski et al., 1999; Randall et al., 2011; Rosen et al., 2011). Specifically, we have tested empirically Burke et al.'s (2006) assumption that adaptive team performance is impossible without SMMs among team members. The investigation of the role of team knowledge and communication in adaptation also differs from recent empirical research that assessed team adaptation in relation to coordination (Summers et al., 2012) and strategy mental models (Randall et al., 2011).

The data reveal that mental model accuracy improves pre- and post-change team performance. This finding extends previous studies on the positive relationship between shared knowledge and team performance (Kozlowski et al., 2009; Langan-Fox et al., 2009; Mathieu et al., 2010; Mohammed et al., 2010; Resick et al., 2010) and provides additional detail to the role of SMMs in adaptive team performance (Baard et al., 2014; Burke et al., 2006; Kozlowski et al., 2009; Rosen et al., 2011). The results of similarity of task- and team-related mental models show that these knowledge structures have no significant effect on performance before or after the unforeseen change. These findings support the idea that effective team members not necessarily need both accurate and similar knowledge structures but that mental model accuracy only can benefit team performance of tasks that can be solved in multiple ways (Resick et al., 2010). Also, they stress the importance to distinguish accuracy from similarity of mental models to understand adaptive team performance (Baard et al., 2014; Burke et al., 2006; Randall et al., 2011; Rosen et al., 2011).

The results add further detail to the notion that mental model accuracy is pivotal in adaptive team performance (e.g. Burke et al., 2006; Randall et al., 2011) and has a function in minimizing initial confusion after a change and in subsequent rapid strategy selection (Rico et al., 2008). However, the present findings imply that the recent suggestion that mental model similarity are not necessarily beneficial to adaptive team performance (Baard et al., 2014; Marks et al., 2001) may be 
true. Team members who have similar knowledge may be unable to create essential and new shared knowledge structures if the altered new situation calls for alternative strategies. The reason for this is that these teams may be less sensitive to detecting and diagnosing changes in their environment and are therefore less flexible to develop new plans and strategies (Uitdewilligen et al., 2010). Based on this reasoning, similar knowledge structures may fail to aid adaptive team performance after an unforeseen change (Audia et al., 2000; Uitdewilligen et al., 2013; Uitdewilligen et al., 2010).

An important question in the present study was whether standardized communication would help teams to sustain communication patterns after the unforeseen partial system failure. We found no effect of standardized communication on minimizing the performance drop after the change and on the reacquisition of skills in the new situation. This is in line with the notion that teams will experience a coordination flux after the change (Summers et al., 2012), which makes it impossible to reapply learned communication skills. The communication protocols applied in our study focused on concise systematic communication pertaining to the geometry of the game pieces. The unforeseen change included a radical change of colour coding of games pieces, added by a partial visual occlusion of the game area on screen. This combination apparently created a coordination flux (Baard et al., 2014; Burke et al., 2006; Randall et al., 2011; Rosen et al., 2011) that sufficed to make standardized communication ineffective. In addition to previous research, which showed the negative effects of task changes on coordination behaviour (Summers et al., 2012), the present study contributes to the literature by implying negative effects on team communication.

The present study stands somewhat apart from most team adaptation studies in that the sudden change we induced pertains to a unique partial system failure (Weick, 1985; Weick \& Roberts, 1993). Such a failure is feared by most organizations (Bainbridge, 1983) and especially when it is costly and difficult to diagnose and repair within a short period of time (Marks et al., 2002). It has been long known that it is hard to train employees to handle low-frequent or even unique failures (Bainbridge, 1983). The present approach adds to this literature as it shows the limits of the potential benefits of teams in terms of shared knowledge and standardized communication. Further research should continue with finding those aspects that significantly aid adaptive team performance, such as individual characteristics or team situation awareness (Burke et al., 2006).

\section{Practical implications}

A number of studies claim that team adaptation training can be efficient in facilitating stable team performance, even in novel situations (e.g. Gorman et al., 2010; Salas, Nichols, \& Driskell, 2007). It is indeed possible that standardized communication training in the present study had certain positive effects as suggested in the literature (Burke et al., 2006; Kanki et al., 1991; Salas, Nichols, et al., 2007) but that these effects were nullified by negative aspects such as cognitive inflexibility (Gersick, 1988; Stachowski et al., 2009; Summers et al., 2012). Possible positive effects of standardized communication refer to the common understanding within a team about the interaction between team 


\section{Chapter 6}

members. This should provide a robust basis that remains usable after the unforeseen change. Potential negative effects of standardized communication include the difficulties that team members have with discarding recently learned patterns even though they are less appropriate in the new situation. Further research is needed to reveal such a potential negation mechanism or confirm that the unforeseen change in the present study caused a large part of the recently acquired knowledge to become redundant and unsuited to effectively adapt to the induced partial system failure.

The present outcomes show that a standardized communication intervention may be quite hard to realize. Organizations often believe that existing communication protocols are adequate for any (also a unique unforeseen) situation, so that adaptation of communication is unnecessary. The present study suggests that such an approach may be rather unrealistic. Unforeseen situations pertaining to unique failures and limitations to automated systems are inherently difficult to predict (Bainbridge, 1983; Weick, 1985; Weick \& Roberts, 1993). This makes it quite difficult to design adequate training to prepare teams for all possible environmental events (Marks et al., 2000). Consistent with this line of thinking is the finding that procedural training, in which team members learn to follow specific procedures, can result in poor adaptation ability in changing and unforeseeable task environments (Gorman et al., 2010).

Other types of interventions may include training in applying strategies to give up formally successful routines and learned behaviour at an appropriate time. Teams should be able to timely appraise the new situation and decide that it asks for new behavioural patterns (Burke et al., 2006; Kraiger, Ford, \& Salas, 1993). These ideas could contribute to further improve crew resource management training, which still has inconsistent effects on learning and behavioural change (Salas, Wilson, Burke, \& Wightman, 2006). A potential instructional method is to use various strategies in a flexible way. The so-called 4C/ID model propagates schema construction for nonrecurrent aspects of the task (Van Merriënboer \& Dijkstra, 1997). Schemas are knowledge structures that enable to apply knowledge and skills effectively and are believed to function in a wide variety of real-life situations. Knowledge contents in a schema are rather abstract, less situation, and context specific and may apply to a variety of known and unknown situations. Future research should investigate the effectiveness of these notions in team adaptation.

\section{Limitations}

The present laboratory study aimed to understand team processes as a preparation to test these in the field. The study therefore had an inherently limited external validity with undergraduate students who worked together on a laboratory team tasks. Specifically, the task environment was not able to fully approach the safety-critical aspects that are common in so-called action team tasks (e.g., ATC). Note, however, that the task was quite complex as teams did not reach an asymptote in their learning curves as it is typical in real teams. In addition, a lack of external validity is inherent to laboratory studies, but these studies are needed to develop a good understanding of team processes before we can test these in 
the field (Zijlstra et al., 2012). Future research should reveal whether the present findings on adaptive team performance can be replicated in field settings. In addition, future research should replicate the study with a larger sample size to confirm the generalizability of the present results.

Another potential limitation of the present study was the restricted period of time. In total the experimental session lasted six times $10 \mathrm{~min}$ of game play. It is possible that the number of trials may have been insufficient to explain performance changes by shared knowledge or by standardized communication training condition. However, a pilot study showed that team performance improved considerably across three trials. And despite the short duration of each trial the results show a clear change in team success over time. This implies that some team processes were effectively at work. In addition, freshly resembled teams may work in comparable settings in which efficient teamwork is required in a short period of time without extensive training possibilities. The present results are specifically relevant for such settings.

Similarly, mental model similarity may not have influenced adaptive team performance because these knowledge structures require time to develop while the present cooperation within a team lasted relatively short (e.g. Mathieu et al., 2010). However, the obtained values for mental model similarity in the present study were not particularly low as compared to other studies. Still, the applied measures of shared knowledge may have been too insensitive to detect differences (Mohammed et al., 2010; Resick et al., 2010). This may pertain not only to the type of measurement but also to what the measure represents. There are various types of measurement. SMMs have been appraised via questionnaires, concept maps, or association ratings (Mohammed et al., 2000). A recent meta-analysis revealed that measurements of the structure and organization of knowledge are most predictive for team processes but that all types of measurements are equal in predicting team performance (DeChurch \& Mesmer-Magnus, 2010b). In predicting team adaptation in decision-making teams, structural network metrics seems to be most valid (Resick et al., 2010). It is however unclear whether this holds in other types of teams. The method used in the present study (cue-strategy association and positional goal interdependency measure) was carefully chosen as it was believed to be appropriate to predict adaptive team performance of action teams in a dynamic environment, in which a number of alternative actions can be effective (Mathieu et al., 2010).

A related limitation is the measurement of mental model accuracy that did not differentiate between task- and teamwork-related aspects. The results imply that the degree to which team members share accurate knowledge in general positively affects adaptive team performance. Future research should however show whether this results also hold true for more specific forms of knowledge accuracy among team members, that is, knowledge that is related to the task or teamwork.

\section{Conclusion}

In sum, this study investigated the discontinuous development of performance through subsequent phases to examine adaptive team performance after an unforeseen change in the task environment. The 


\section{Chapter 6}

results confirm that adaptive team performance is a highly complex process (Burke et al., 2006; Entin \& Serfaty, 1999; Kozlowski et al., 2009; Rosen et al., 2011) and add a unique insight to team adaptation research. The results specifically suggest that a team's knowledge accuracy may benefit performance in all subsequent phases of team adaptation. Knowledge similarity and recently acquired standardized communication protocols, which are generally thought to be beneficial for team performance (Burke et al., 2006; Entin \& Serfaty, 1999; Kozlowski et al., 2009; Randall et al., 2011; Rosen et al., 2011), are not necessarily adequate if the task environment suddenly changes in unforeseen and unique ways. To make adaptive team performance to these changes successful a team needs to train in updating shared knowledge and standardized communication beyond the change (Audia et al., 2000; Gersick, 1988; Stachowski et al., 2009; Uitdewilligen et al., 2013) and to quickly recuperate and develop new knowledge and strategies to regain maximum performance. 


\section{Chapter 7}

\section{General discussion and conclusion}





\section{The Contribution of this Dissertation}

Passengers on board an aircraft expect to travel comfortably to their destination and assume high standards of safety with no delays of departure or arrival. Teams of highly competent individuals contribute towards fulfilling these needs for safety and comfort. Particularly, smooth cooperation between team members can make a difference in safe and efficient air transportation (Malakis et al., 2010b). Effective teamwork in complex and dynamic environments, such as in air traffic control (ATC), is a precondition for success and therefore continuously subjected to the need for improvement (Fiore et al., 2010). The present dissertation studied teamwork by means of the concept of shared mental models with the purpose to give insight into the shared knowledge structures of air traffic controllers (ATCOs) in particular and into the role of shared mental models in action teams in general.

This dissertation extended our knowledge on this topic by addressing three research aims. The first aim was to refine the shared mental model construct in an operational context, namely in the domain of ATC. The second research aim included revealing the role of knowledge sharing in developing shared mental models. Finally, the third research aim focused on investigating the relationship between shared mental models, communication and adaptive team performance in response to an unforeseen event. These aims took into account the Two-Level Approach of Shared Mental Model outlined in the introduction. Consequently, shared mental models were assessed on both the reflectional level and on the action level. The reflectional level was approached by interviewing ATCOs and studying formally documented knowledge requirements. In addition, shared mental models were assessed on the action level, by studying shared mental models of student teams during task execution via scenario-based measurement and communication analysis. Mixed methods of qualitative, quasi-experimental and experimental study designs were used to assess shared mental model on both levels.

\section{Theoretical Implications}

Early and current notions about shared mental models include that multiple types of shared mental models exist (Cannon-Bowers et al., 1993; Klimoski \& Mohammed, 1994). These notions have theoretical value but to date they both lack empirical evidence and applied confirmation (Lim \& Klein, 2006; Mohammed et al., 2010). The present dissertation indicates that teams in the field tend to share a number of types of mental models (Chapter 2). However, a generic typology of these shared mental model types (e.g. Cannon-Bowers et al., 1993) may be insufficient in representing shared knowledge of these teams. Teams in the field have specific knowledge requirements and a typology that described their shared mental models should gain detail via domain and team specific elements (Mohammed et al., 2010). The framework developed in the present research contributes to the theory as it gives a detailed description of these types of shared mental models in terms of their knowledge elements and characteristics. 
Specifically, in the domain of ATC, shared mental models of the situation and the equipment are critical. These types of knowledge seem relevant in many dynamic and system-driven team environments such as offshore oil industry, nuclear power plants, or surgery rooms (Waller et al., 2004). However, most researchers ignore these types of shared mental models (Gross \& Kluge, 2014; Lim \& Klein, 2006; Mathieu et al., 2010). The present dissertation therefore refines shared mental model theory by showing that typologies describing shared knowledge of complex teams in the field should include shared situation and shared equipment knowledge.

This research also refines shared mental model theory through the identification of two dimensions of shared mental models in ATC (Chapter 3). These dimensions distinguish content (task/equipment versus team) and dynamic (dynamic versus static) of shared knowledge and confirms earlier notions in shared mental model theory. These notions suggest that shared knowledge can be distinguished in task- and team-related aspects (Klimoski \& Mohammed, 1994; Mathieu et al., 2000) and that the content of some types of shared mental models varies as a function of task demands and situational parameters (Cannon-Bowers et al., 1993). Those shared mental model types that vary with task demands and situational parameters presumably require different assessments, for example ontask observations, than static shared mental model types. The identified dynamic dimension may therefore help researchers to choose appropriate measurement methods when studying different types of shared mental models.

The studies presented in this dissertation identified that especially accuracy and not the similarity of shared knowledge had a positive role in team performance (Chapters $4 \& 5$ ). This finding contributes to the shared mental model literature as there is still no consensus about the comparative validity of mental model similarity and accuracy in team performance. While most researchers agree that mental model similarity is a stronger predictor of team performance (Marks et al., 2000; Mathieu et al., 2010; Webber et al., 2000), some studies also suggest that accuracy contributes most to team performance (Edwards et al., 2006; H. H. McIntyre \& Foti, 2013). Accuracy may be more important to team performance in tasks in which a number of strategies are effective to perform successfully, as it was the case in the present TeamTris task. In such a task context, a team may benefit most when its team members have a variety of accurate knowledge structures that all contribute to effective team performance. Contrary, it may be ineffective or redundant that all team members know the same task strategies. Sharing mental models would then imply dividing knowledge among team members about the many possible task strategies (Mohammed et al., 2010) and this would make the team effective.

Mental model accuracy also plays an important role in team performance before and after an unforeseen change in the task environment (Chapter 6). Specifically, this dissertation demonstrated that team members with accurate mental models generally perform better than team members with less accurate mental models even if the system used partially breaks down. This finding provides additional detail to the role of accurate mental models in adaptive team performance (Baard et al., 2014; Kozlowski et al., 2009; Rosen et al., 2011). 
However, we also proofed the limits of the potential benefits of similar mental models and standardized communication in adaptive team performance (Chapter 6). Mental model similarity and standardized communication did not help team members to adapt more effectively. This contradicts the conceptual model of team adaptation of Burke and co-workers that claims that adaptive team performance is only possible if team members have a certain basis of shared knowledge (Burke et al., 2006; Randall et al., 2011). Also, the model predicts that communication has an essential role in adaptive behaviour. However, similar mental models and standardized communication may be particular helpful in less severe changes in the task environment. The unforeseen partial system breakdown introduced in the present research, however, made learned strategies unworkable and led to coordination flux (Summers et al., 2012). Consequently, teams had to rebuild shared knowledge and had to adapt their communication patterns. It is possible that the type and severity of change is a determining factor in the role of shared mental models in team adaptation.

Few studies have investigated potential means to encourage the development of shared mental models. Therefore, this dissertation attempted to provide more insight into facilitating shared mental models through knowledge sharing (Chapters $4 \& 5$ ). The results of this dissertation confirm that information exchange is a key element to shared mental model development that should be encouraged and enabled (Kennedy \& McComb, 2010; Langan-Fox et al., 2004). Our studies demonstrated that team members benefit from additional opportunities for information exchange during knowledge sharing about aspects of both the task and the team functioning. This finding empirically extends the theoretical notion that team members develop shared mental models of the task before they develop shared mental models of the team (Kennedy \& McComb, 2010; McComb, 2007). We could show that knowledge sharing is effective when it is designed according to this gradual development of shared mental models from task to team knowledge.

Finally, we found comprehensive results regarding team behaviour and specifically communication. Team members who communicate more task- and team-related information developed more effective shared mental models (Chapter 4). Through communication team members managed to verbalize their intentions and explicitly shared their knowledge. While it takes time and mental capacity to communicate, this additional investment of resources early in a team's lifecycle will help the team members later on (Fischer, McDonnell, \& Orasanu, 2007; Zijlstra et al., 2012). The present results also imply that communication affects shared mental model development when the communication pertains to topics that were addressed earlier. This confirms ideas of the shared mental model literature that shared mental models develop iteratively (Kennedy \& McComb, 2010; McComb, 2007). Communication during task execution was a repetition of information that team members had already shared before task performance (e.g. during a team discussion).

An associated communication behaviour of shared mental model development was closedloop communication (Chapter 5). Team members who confirmed and cross-checked information also shared more similar and more accurate mental models. This results confirm that closed-loop 


\section{Chapter 7}

communication indicates the presence of team members shared mental models (Salas, Rosen, et al., 2007). However, also mutual performance monitoring is believed to be an indicator of shared mental models (Salas, Rosen, et al., 2007), but this dissertation shows that this is not always the case. The task environment may hamper specific team behaviours even if team members have effective shared mental models. For example, in the task environment applied in the present studies, it was less beneficial to engage in mutual performance monitoring as it distracted team members from task execution. Consequently, although team members had developed accurate and reasonably similar mental models they did not show more mutual performance monitoring. Consequently, notions on associated behaviours of shared mental models should specify the task context as team behaviours may not be an indicator of shared mental models in specific task. Moreover, researcher and practitioners should be careful with their conclusion on team members' shared mental models in the absence of team behaviours.

\section{Practical Implication}

The present domain-specific analysis of shared mental models has implications for teamwork among ATCOs. The detailed distinction of five shared mental model types (equipment, task, team, team interaction and situation) can help to explain when team members efficiently work together. Instructors and assessors may use this distinction in an ATC training setting to diagnose performance problems in the teamwork of ATC trainees. These performance problems can arise from insufficiently shared knowledge. A detailed understanding of the types of shared mental models can help assessors to pinpoint the type of knowledge that is insufficiently shared among team members. Consequently, assessors can provide remedial training that are tailored to the knowledge needs of the trainee(s).

A training tailored to needs of the trainee should present the relevant information in a simplified format so that the information is meaningful to the trainees. This is because the present research shows that experienced ATCOs in the field are less detailed in the description of their shared knowledge. ATCOs are unable to differentiate between certain types of shared mental models and instead often combine these types in broader categories. For example, one broader category entails shared knowledge of task and situation. Another category entails shared knowledge of team and team interaction. Thus, when training ATCOs in shared knowledge development, knowledge types that belong to of one broader category should be presented in combination.

The present dissertation may also help in choosing a suitable training form to train shared mental models efficiently. Shared mental model types that have been identified as dynamic, this is, which content frequently alters in response to changes in the environment, require different team training strategies as compared to shared mental model types that are relatively stable over time (Van Merriënboer \& Dijkstra, 1997). Stable shared mental model types, such as equipment knowledge, may be easily integrated in existing knowledge structures (Kirschner \& Van Merriënboer, 2008) and could be trained early in an education program in a classroom setting. Dynamic shared mental models types, 
such as team knowledge, may require a more intense training form in a team setting. Scenario-based training could be a successful training form because it offers a variety of situations in a realistic environment (Cannon-Bowers, Burns, Salas, \& Pruitt, 1998).

In addition to training, this dissertation implies that organizations should provide opportunities for knowledge sharing during daily operations to improve team performance. Specifically, knowledge sharing during regular debriefings are a promising strategy to increase effective team behaviour, shared mental models and team performance. While debriefing is not unusual in aviation, these are often unstructured and primarily carried out after an incident. Therefore, debriefings in form of guided team discussions should be more rigorously implemented in the daily work of ATCOs. These guided discussions should cover both general aspects of teamwork, such as the team goal, task strategies and the distribution of workload, as well as an evaluation of situation specific events. Organizations could support these team discussions by providing debriefing sheets that lead team members through important topics and that structure the discussions.

Organizational support for shared mental model development could be particular important in future air traffic management. There are two main reasons. First, the harmonization of European airspace through the Single European Sky programme will require team members of different nations to closely work together (Neal et al., 2011). These team members have different cultural and training backgrounds and will in many cases be unfamiliar with each other. They may have different understandings about task strategies and little knowledge of each other's skills and attitudes. Encouraging the development of shared mental models through knowledge sharing during guided team discussions would therefore become increasingly important for these future teams. Second, datalink communication will replace voice-communication in future air traffic management (LanganFox et al., 2009). During datalink communication team members have restricted possibilities for personal interaction and information exchange and this may hamper the development of their shared mental models. Guided team discussions and proper debriefings can help to overcome some of these potential difficulties and help team members to develop shared knowledge in a datalink environment.

Organizations can support shared mental model development without negatively affecting team members' ability to adapt to an unforeseen change. This implication grounds on the present finding that shared mental models had neither a positive nor a negative effect on team adaptation. Previous studies have warned against the potential negative effects of shared mental models on team adaptation (e.g. Audia et al., 2000; Uitdewilligen et al., 2013) and this would present organizations with a dilemma between the positive effects of shared mental models for general team performance and the negative effects for adaptive team performance.

In contrast, organizations face a dilemma regarding standardized communication as standardized communication has costs and benefits. The benefit is that team members can communicate concisely in regular situations with little risks for misunderstanding. The costs are that they are less flexible in situations that require alternate communication patterns. These often 


\section{Chapter 7}

unforeseen situations lead to a coordination flux (Summers et al., 2012) that team members must overcome through learning new coordination strategies to regain team efficiency. Training should therefore be directed towards strengthening the benefits of learned routines and weakening the costs of inflexibility. These ideas could contribute to further improve and refine crew resource management training, which still has ambiguous effects on learning and behavioural change (Salas et al., 2006). Specifically, training instructors could train team members to be aware and take advantage of the relative predictable behaviours of team members, since they are communicating in a standardized way. In addition, team members should learn to recognize changes in the task environment early and adapt their behaviour accordingly (Burke et al., 2006; Kraiger \& Wenzel, 1997; Van Merriënboer \& Dijkstra, 1997).

\section{Future Research}

A number of specific suggestions for future research has been included in the individual studies of this dissertation. This section therefore elaborates on general suggestions for future research that excel in conducting this research.

First, this dissertation can inspire further research in ATC. For example, shared mental models should also be studied in larger cooperative teams, which consist of several sub-teams. In air traffic management these are for example the air navigation service provider, cockpit, airport and airline. The process of inter-team shared mental models is particularly interesting in these teams as cooperation and collaborative decision making between sub-teams becomes increasingly critical in air traffic management (Neal et al., 2011). It is essential that they have access to and share the same information to be able to quickly agree on a decision. Conversely, individual sub-teams might also try to hold back information to their advantage. The mechanisms and effects of shared mental models of such teams might look very different to those of intra-team shared mental models studied so far.

Observation during experimental sessions inspires another possibility for future research, namely the need for shared mental models in teams with a highly competent team member who takes the lead (a so-called peer leader). In this research some successful teams included a team member, who was very skilled and quickly understood the task strategies. Often this team member gave instructions to the others on how to perform their tasks and decided which task strategies the team applied. While these teams might have developed a shared understanding, the knowledge and skills of the leading team member presumably drove this shared understanding. Two questions arise that need attention in future studies. First, whether the process of shared mental model development in teams having a peer leader differs from that found in teams without a peer leader; and second, whether the importance of shared knowledge structures decreases if one highly competent individual takes responsibility for task execution, while the other team members merely follow this individual's instructions. It might be possible that in such situations a shared mental model is redundant. Previously published work demonstrates that different types of leadership can indeed affect mental 
model convergence, but have different effects on team performance (Dionne, Sayama, Hao, \& Bush, 2010). Beside this study, research into the relationships between leadership, shared mental model development and team performance is scarce, and should be carried out more extensively in the future.

Final recommendations for future research pertain to the methods that have been proved useful in this dissertation. First, the combination of card sorting and permutation testing (Good, 2000) was effective in studying differences in mental model similarity. The addition of permutation methods allows to compare mental model similarity of different teams, while card sorting captures the content of shared mental models (DeChurch \& Mesmer-Magnus, 2010b). Second, discontinuous growth modelling successfully allowed the detailed investigation and visualisation of transition adaptation and reacquisition adaptation in teams. Discontinuous growth modelling has only been applied to investigate adaptation in individuals (Lang \& Bliese, 2009), while in this dissertation we also showed that teams run through the same adaptive phases when they are exposed to an unexpected change by applying this technique.

\section{Conclusion}

The studies in this dissertation gave insights into the role and development of shared knowledge in action teams. Specifically, the shared mental models of ATCOs were analysed to build a domain specific framework of shared mental models in ATC. This framework can be applied to predict and explain team behaviour, and to design training strategies that are tailored to those shared mental model types that are important in ATC. Furthermore, the results presented empirical confirmation of the theoretical literature that multiple types of shared mental models exist in the task domain of ATC. Mental model similarity and accuracy were assessed during both normal and unforeseen situations. The acknowledged positive effects of shared mental models under normal circumstances did not help adaptive team performance. In normal circumstances, knowledge sharing is an important facilitator of shared mental model development and subsequent team performance. Providing team members with possibilities for knowledge sharing can help to increase the accuracy of team member's mental models, especially in environments in which explicit information sharing is limited. I hope that the insights generated in this research will find their way to the operational room and will contribute to safe aviation in the future. 



\section{Summary}

Air traffic control and other highly dynamic and complex work environments require team members who effectively work together. Specifically, shared knowledge between team members helps air traffic controllers to jointly work towards a common goal. This shared knowledge is also referred to as a shared mental model, which is the common knowledge between members of a team that help them to coordinate their actions and work together effectively. This dissertation aims to increase our knowledge of shared mental models in complex teams and specifically teams of air traffic controllers to better understand how teamwork can be improved. Specifically, this dissertation addressers three research aims, namely (1) refine the shared mental model construct in the context of air traffic control, (2) reveal the role of knowledge sharing in developing shared mental models and (3) investigating the relationship between shared mental models, communication and adaptive team performance in response to an unforeseen change.

Chapter 2 contributes to the first research aim and refines the shared mental model construct in air traffic control. By means of a cognitive task analysis the shared knowledge structures of air traffic controllers are analysed. This analysis includes qualitative data collection methods (interviews and observations) that result in a framework of shared mental models of air traffic controllers. The framework comprises of five shared mental model types, namely equipment, task, team, team interaction and situation. A number of knowledge elements that specify the content of these shared mental models describe each type in more detail. Particularly the situation model constitutes an important difference to existing mental model typologies and emphasizes the dynamic nature of the task of air traffic controllers. This first study demonstrates the need to refine shared mental models within a specific domain. The framework developed constitutes the basis for the subsequent study.

Chapter 3 elaborates on the first research aim by testing the validity of the framework for 100 controllers. In addition, this study compares the similarity of the shared mental models identified between controllers who work for separate organizations and with different levels of work experience. Air traffic controllers conduct a card sorting task during which they categorize knowledge elements (cards) into predefined categories (shared mental model types). Multi dimensional scaling reveals a marked deviation from the proposed five types of shared mental models of the framework. Air traffic controllers distinguish three types of knowledge, namely related to the equipment, the task, and the team. Subsequent permutation tests shows that mental model similarity of task-related and equipment knowledge varies as a function of organizational context and work experience. It appears that the organizational context affects the similarity of air traffic controllers' mental models, but that this effect diminishes with increasing work experience. This study highlights that team members categorize knowledge in meaningful chunks and that a theoretical supported framework, as often presented in the 
literature, as well as suggested in this dissertation, is not directly applicable in the field. In addition, the findings contributes to the literature as it shows that both external (organization) and internal (work experience) aspects can influence team members shared mental models.

Chapter 4 addresses the second research aim, which regards the role of knowledge sharing in developing shared mental models ${ }^{7}$. To test this, three-person teams carry out a dynamic team task TeamTris - that have been developed to simulated the main principles of complex and dynamic environments. Half of the teams receive guided and off-task opportunities for knowledge sharing through (a) exchanging information about each other's tasks and (b) a team discussion. Results show that knowledge sharing positively influences mental model accuracy and the similarity of team-related mental models. Mental model accuracy in turn predicts team performance. In addition, knowledge sharing positively correlates with the amount of task- and team-related communication during task performance and this further improves shared mental model development.

Chapter 5 further elaborates on the second research aim and investigates the development of shared mental models in a datalink setting. Datalink is increasingly prevalent in air traffic control and other complex environments. It allows exchanging big amounts of data but also reduces information exchange to predefined messages, which may hamper the development of shared mental models. This study therefore tests the effects of knowledge sharing opportunities on shared mental model development and on team behaviours associated with shared mental models. Knowledge sharing is operationalize as (a) information exchange about each other's tasks, (b) a team discussion and (c) hands-on experience with the tasks of other team members. Results demonstrate that knowledge sharing positively influence the team behaviour closed-loop communication, as well as mental model accuracy and the similarity of team-related mental models. In line with the outcomes of the previous study, mental model accuracy predicts team performance.

Chapter 4 and Chapter 5 show that off-task opportunities for the mutual exchange of information contribute to converge individual knowledge into shared knowledge among team members. Consequently, knowledge sharing is a potential means for organizations to support team members in the development of effective shared mental models. The two studies also confirm the close relationship between shared mental models and communication. Communication both reinforces shared mental models and indicates their presence.

Chapter 6 establishes insights on the third research aim by studying the relationship between shared mental models, communication and adaptive team performance. Adaptive team performance is particularly critical in dynamic environments such as air traffic control as situations frequently change and team members need to adapt to these changes. This study investigates how team members perform in response to an unforeseen change (partial system breakdown) and whether shared mental models

\footnotetext{
${ }^{7}$ Based on the results of the first two studies subsequent studies focused on task-related and team-related shared mental models. The equipment model type was excluded because the equipment of the studied task environment was rather simple so that little need for team members to share knowledge of the equipment was expected.
} 
and standardized communication help teams to adapt to the change. Results of discontinuous growth modelling analysis show that team performance drops immediately after the change and then slowly increases again to the initial level. This performance pattern is the same for all teams irrespective of whether they are trained in standardized communication protocols, or whether they have more similar and accurate mental models. However, team members with highly accurate mental models perform generally better before and after the change. This study implies that unforeseen changes disrupt team members' shared mental models and developed communication patterns. The shared mental models and communication patterns that team members develop in a given situation become useless in a new situation that requires different task- and cooperation strategies.

Based on the results of this research it can be concluded that team members in the field share multiple types of mental models. In addition to general shared mental model types of aspects of task and team, shared mental models of the situation and equipment are critical in dynamic and systemdriven environments. Additionally, this research shows that organizations can support team members in the development of shared mental model through providing additional opportunities for knowledge sharing. Knowledge sharing improves team members' shared knowledge structures and particularly accurate knowledge structures between team members contribute to team performance. These shared knowledge structures, however, do not help teams to adapt to an unforeseen change. 



\section{Samenvatting}

Luchtverkeersleiding en andere zeer dynamische en complexe werkomgevingen hebben teamleden nodig die effectief samenwerken. Vooral de gedeelde kennis tussen teamleden helpt luchtverkeersleiders om gezamenlijk naar één doel toe te werken. Deze gedeelde kennis is ook bekend als 'shared mental model' of gedeelde mentale model, gedefinieerd als de gemeenschappelijke kennis tussen leden van een team, die hun helpt om acties te coördineren en om effectief samen te werken. Dit proefschrift heeft als doel om de kennis over gedeelde mentale modellen in complexe teams en vooral in teams van luchtverkeersleiders te vergroten om zodoende beter te kunnen begrijpen hoe teamwerk verbeterd kan worden. Specifiek behandelt dit proefschrift drie onderzoeksdoelen: (1) het construct van gedeelde mentale modellen in de context van luchtverkeersleiding verfijnen, (2) de rol van kennisdeling bij het ontwikkelen van gedeelde mentale modellen te achterhalen en (3) de samenhang tussen gedeelde mentale modellen, communicatie en adaptieve teamprestatie na een onverwachte verandering in de taakomgeving te onderzoeken.

Hoofdstuk 2 draag bij aan het eerste onderzoeksdoel en verfijnt het construct van gedeelde mentale modellen in luchtverkeersleiding. Door middel van een cognitieve taakanalyse worden de gedeelde kennisstructuren van luchtverkeersleiders geanalyseerd. Deze analyse omvat methoden van kwalitatieve datacollectie, zoals interviews en observaties, en resulteert in een raamwerk voor gedeelde mentale modellen van luchtverkeersleiders. Het raamwerk bevat vijf types gedeelde mentale modellen, namelijk apparatuur, taak, team, team interactie en situatie. Elk type wordt beschreven door een aantal kenniselementen dat de inhoud van deze gedeelde mentale modellen specificeert.

Voornamelijk het situatie model is een belangrijk verschil met bestaande typologieën en benadrukt de dynamische aard van het werk van luchtverkeersleiders. Deze eerste studie laat daarom de noodzaak zien om gedeelde mentale modellen in een specifiek domein te onderzoeken. Het ontwikkelde raamwerk vormt de basis voor de volgende studie.

Hoofdstuk 3 gaat verder op het eerste onderzoeksdoel in door de validiteit van heet raamwerk voor 100 luchtverkeersleiders te testen. Daarnaast vergelijkt dit hoofdstuk de overeenkomsten tussen de geïdentificeerde gedeelde mentale modellen van luchtverkeersleiders, die voor verschillende organisaties werken en die verschillend niveau van werkervaring hebben. Luchtverkeersleiders voeren een 'card sorting' taak uit waarbij ze kenniselementen (kaarten) in vooraf gedefinieerde categorieën moeten verdelen (types gedeelde mentale modellen). Multi-dimensional scaling laat een markant verschil van de eerder geïdentificeerde types gedeelde mentale modellen zien. Luchtverkeersleiders onderscheiden drie types van kennis die gerelateerd zijn met apparatuur, de taak, en het team. Daaropvolgende permutatietests tonen aan dat overeenkomsten tussen taak-gerelateerde kennis en kennis over apparatuur verschillen met betrekking tot organisatie en werkervaring. De 
organisatiecontext beïnvloedt de overeenkomst tussen mentale modellen van luchtverkeersleiders, maar dit effect neemt met toenemende werkervaring af. Deze studie laat zien dat teamleden hun kennis in betekenisvolle 'chunks' categoriseren en dat een theoretisch beschreven raamwerk, zo als vaak in de literatuur, als ook in dit proefschrift, in de praktijk niet direct toepasbaar is. Daarnaast dragen deze bevindingen bij aan de literatuur door aan te tonen dat externe (organisatie) en interne (werkervaring) factoren de overeenkomsten van mentale modellen tussen teamleden kunnen beïnvloeden.

Hoofdstuk 4 pakt het tweede onderzoeksdoel aan, dat de rol van kennisdeling bij de ontwikkeling van gedeelde mentale modellen ${ }^{8}$ onderzoekt. Hiervoor voeren teams van drie personen een dynamische teamtaak uit - TeamTris - welke ontwikkeld is om de hoofdprincipes van complexe en dynamische taakomgevingen te simuleren. De helft van de teams krijgt voorafgaand aan de taakuitvoering de mogelijkheid voor kennisdeling door (a) informatie over elkaars taken uit te wisselen en (b) een teamdiscussie te voeren. De resultaten laten zien dat kennisdeling de accuraatheid van mentale modellen en de overeenkomsten van team-gerelateerde mentale modellen positief beïnvloedt. De accuraatheid van mentale modellen is vervolgens voorspellend voor teamprestatie. Daarnaast correleert kennisdeling positief met de hoeveelheid communicatie over taak- en teamaspecten tijdens de takkuitvoering. Dit verbetert vervolgens weer de ontwikkeling van de gedeelde mentale modellen.

Hoofdstuk 5 gaat nog verder in op het tweede onderzoeksdoel en onderzoekt de ontwikkeling van gedeelde mentale modellen in een datalink omgeving. Datalink komt steeds meer voor bij de luchtverkeersleiding en andere complexe werkomgevingen. Door middel van datalink kunnen grote hoeveelheden data uitgewisseld worden. Datalink reduceert echter informatie-uitwisseling tot vooraf gedefinieerde berichten en dit kan de ontwikkeling van gedeelde mentale modellen mogelijk belemmeren. Deze studie test daarom het effect van de mogelijkheid tot kennisdeling op de ontwikkeling van gedeelde mentale modellen en op teamgedrag dat met gedeelde mentale modellen geassocieerd is. Kennisdeling vindt plaats door middel van (a) informatie over elkaars taken uit te wisselen, (b) een teamdiscussie te voeren en (c) praktische ervaring met elkaars taken op te doen. De resultaten demonstreren dat kennisdeling het teamgedrag 'closed-loop communication', zoals de accuraatheid van mentale modellen en de overeenkomsten van team-gerelateerde mentale modellen positief beïnvloedt. In overeenstemming met de vorige studie voorspelt de accuraatheid van mentale modellen teamprestatie.

Hoofdstuk 4 en Hoofdstuk 5 laten zien dat additionele mogelijkheden voor wederzijds informatie-uitwisseling helpen om individuele kennis te convergeren naar gedeelde kennis tussen teamleden. Kennisdeling is derhalve een potentieel middel voor organisaties om teamleden bij de ontwikkeling van effectieve gedeelde mentale modellen te ondersteunen. Daarnaast bevestigen de

\footnotetext{
${ }^{8}$ Gebaseerd op de resultaten van de eerste twee studies onderzoeken de volgende studies taak- en teamgerelateerde gedeelde mentale modellen. Het apparatuur model wordt niet verder beschouwd omdat de taakomgeving in de studies een versimpelde form van de realiteit is en uitgebreide kennis over de apparatuur daarbij niet nodig is.
} 
twee studies de nauwe relatie tussen gedeelde mentale modellen en communicatie. Communicatie versterkt zowel gedeelde mentale modellen als kenmerkt diens aanwezigheid.

Hoofdstuk 6 verzamelt inzichten omtrent het derde onderzoeksdoel door de relatie tussen gedeelde mentale modellen, communicatie en adaptieve teamprestatie te bestuderen. Adaptieve teamprestatie is vooral in dynamische omgevingen zoals de luchtverkeersleiding belangrijk, omdat situaties vaak veranderen en teamleden hierop efficiënt moeten reageren. Deze studie onderzoekt hoe teamleden in reactie op een onverwachte verandering (een partiële systeemstoring) reageren en of gedeelde mentale modellen en gestandaardiseerde communicatie ervoor kunnen zorgen dat teams zich beter kunnen aanpassen aan de verandering. De resultaten van de discontinuous growth modelling analyse tonen aan dat teamprestatie meteen na de verandering daalt en dan langzaam weer tot het initiële niveau stijgt. Dit patroon van teamprestatie is gelijk voor alle teams, onafhankelijk of deze teams getraind zijn in het gebruik van gestandaardiseerde communicatie en of deze teams gedeelde en accurate mentale modellen hebben. Wel presteren teams met accurate mentale modellen over het algemeen beter, voor en na de verandering. Deze studie impliceert dat onverwachte veranderingen de gedeelde mentale modellen van teamleden en hun communicatiepatronen verstoort. De gedeelde mentale modellen en de communicatie patronen die teamleden in een bepaalde situatie ontwikkelen worden onbruikbaar in een nieuwe situatie welke andere taak- en coöperatiestrategieën vergt.

Gebaseerd op de resultaten van dit onderzoek kan men concluderen dat teamleden in de praktijk verscheidene mentale modellen delen. Naast de algemene types gedeelde mentale modellen over de taak en het team, zijn in dynamische en systeem-georiënteerde omgevingen gedeelde mentale modellen over de situatie en het equipment belangrijk. Daarnaast laat dit onderzoek zien dat organisaties teamleden in de ontwikkeling van gedeelde mentale modellen kunnen ondersteunen door mogelijkheden voor kennisdeling te creëren. Kennisdeling verbetert de gedeelde kennisstructuren van teamleden en voornamelijk accurate kennisstructuren tussen teamleden dragen bij tot teamprestatie. Deze gedeelde mentale modellen helpen teams echter niet om zich beter aan een onverwachte verandering aan te passen. 



\section{References}

Abma, H., Van Bavelgem, J., Cromie, S., Liston, P., \& Ward, M. (2004). Training needs analysis for human factors practitioners in aviation maintenance. (NLR-CR-2004-223). Amsterdam: National Aerospace Laboratory.

Ackerman, P. L. (1992). Predicting individual differences in complex skill acquisition: Dynamics of ability determinants. Journal of Applied Psychology, 77(5), 598-614. doi:10.1037/00219010.77.5.598

Anderson, J. R. (1981). Cognitive skills and their acquisition. Hillsdale, NJ: Lawrence Erlbaum.

Andres, H. P. (2012). Technology-mediated collaboration, shared mental model and task performance. Journal of Organizational and End User Computing, 24, 64-81. doi:10.4018/joeuc.2012010104

Arrow, H., McGrath, J. E., \& Berdahl, J. L. (2000). Small Groups as Complex Systems: Formation, Coordination, Development, and Adaptation. Thousand Oaks, CA: Sage.

Audia, P. G., Locke, E. A., \& Smith, K. G. (2000). The paradox of success: An archival and a laboratory study of strategic persistence. Academy of Management Journal, 43, 837-853. doi:10.2307/1556413

Auerbach, C., \& Silverstein, L. B. (2003). Qualitative data: An introduction to coding and analysis. New York: New York University Press.

Baard, S. K., Rench, T. A., \& Kozlowski, S. W. J. (2014). Performance adaptation: A theoretical integration and review. Journal of Management, 40(1), 48-99. doi:10.1177/0149206313488210

Badke-Schaub, P., Neumann, A., Lauche, K., \& Mohammed, S. (2007). Mental models in design teams: a valid approach to performance in design collaboration? CoDesign, 3, 5-20. doi:10.1080/15710880601170768

Bainbridge, L. (1983). Ironies of automation. Automatica, 19, 775-779. doi:10.1016/00051098(83)90046-8

Banks, A. P., \& Millward, L. J. (2000). Running shared mental models as a distributed cognitive process. British Journal of Psychology, 91(4), 513-531. doi:10.1348/000712600161961

Banks, A. P., \& Millward, L. J. (2007). Differentiating knowledge in teams: The effect of shared declarative and procedural knowledge on team performance. Group Dynamics: Theory, Research, and Practice, 11, 95-106. doi:10.1037/1089-2699.11.2.95

Barnes, C. M., Hollenbeck, J. R., Wagner, D. T., DeRue, D. S., Nahrgang, J. D., \& Schwind, K. M. (2008). Harmful help: The costs of backing-up behavior in teams. Journal of Applied Psychology, 93, 529-539. doi:10.1037/0021-9010.93.3.529

Bartol, K. M., \& Srivastava, A. (2002). Encouraging knowledge sharing: The role of organizational reward systems. Journal of Leadership and Organization Studies, 9(1).

Blickensderfer, E., Cannon-Bowers, J. A., \& Salas, E. (1998). Cross-training and team performance. In J. A. Cannon-Bowers \& E. Salas (Eds.), Making decisions under stress: Implications for individual and team training (pp. 299-311). Washington, DC: American Psychological Association.

Blickensderfer, E. L., Reynolds, R., Salas, E., \& Cannon-Bowers, J. A. (2010). Shared expectations and implicit coordination in tennis doubles teams. Journal of Applied Sport Psychology, 22. doi:10.1080/10413200.2010.507497

Bliese, P. D. (2000). Within-group agreement, non-independence, and reliability: Implications for data aggregation and analysis. In K. J. Klein \& S. W. J. Kozlowski (Eds.), Multilevel theory, research, and methods in organizations: Foundations, extensions, and new directions (pp. 349 - 381). San Francisco, CA, US: Jossey-Bass.

Bliese, P. D., \& Ployhart, R. E. (2002). Growth modeling using random coefficient models: Model building, testing, and illustration. Organizational Research Methods, 5, 362-387.

doi: $10.1177 / 109442802237116$ 
Bolstand, C. A., \& Endsley, M. R. (1999). Shared mental models and shared displays: An empirical evaluation of team performance. Paper presented at the 43rd Meeting of the Human Factors \& Ergonomics Society.

Borko, H., \& Livingston, C. (1989). Cognition and improvisation: Difference in mathematics instruction by expert and novice teachers. American Educational Research Journal 26, 473498. doi:10.2307/1162861

Brannick, M. T., Salas, E., \& Prince, C. (Eds.). (1997). Team performance assessment and measurement: Theory, methods, and applications. Mahwah, NJ, US: Lawrence Erlbaum Associates Publishers.

Burke, C. S., Stagl, K. C., Salas, E., Pierce, L., \& Kendall, D. (2006). Understanding team adaptation: A conceptual analysis and model. Journal of Applied Psychology, 91, 1189-1207. doi:10.1037/0021-9010.91.6.1189

Cannon-Bowers, J. A., Burns, J. J., Salas, E., \& Pruitt, J. S. (1998). Advanced technology in scenariobased training In J. A. Cannon-Bowers \& E. Salas (Eds.), Making decisions under stress: Implications for individual and team training (pp. 365-374). Washington, DC: American Psychological Association.

Cannon-Bowers, J. A., \& Salas, E. (1998). Individual and team decision making under stress: Theoretical underpinnings. In J. A. Cannon-Bowers \& E. Salas (Eds.), Making decisions under stress: Implications for individual and team training. (pp. 17-38). Washington, DC, US: American Psychological Association.

Cannon-Bowers, J. A., \& Salas, E. (2001). Reflections on shared cognition. Journal of Organizational Behavior, 22, 195-202. doi:10.1002/job.82

Cannon-Bowers, J. A., Salas, E., Blickensderfer, E., \& Bowers, C. A. (1998). The impact of crosstraining and workload on team functioning: A replication and extension of initial findings. Human Factors, 40, 92-101. doi:10.1518/001872098779480550

Cannon-Bowers, J. A., Salas, E., \& Converse, S. (1993). Shared mental models in expert team decision making. In N. Castellan (Ed.), Individual and group decision making (pp. 221-246). Hillsdale, NY: Erlbaum.

Cannon, M. D., \& Edmondson, A. C. (2001). Confronting failure: Antecedents and consequences of shared beliefs about failure in organizational work groups. Journal of Organizational Behavior, 22, 161-177. doi:10.1002/job.85

Chan, D. (2000). Understanding adaptation to changes in the work environment: Integrating individual difference and learning perspectives. In G. R. Ferris (Ed.), Research in personnel and human resources management (pp. 1-42)). Oxford, United Kingdom: JAI Press/Elsevier.

Cohen, J. (1960). A coefficient of agreement for nominal scales. Educational and Psychological Measurement, 20, 37-46. doi:10.1177/001316446002000104

Conger, A. J. (1980). Integration and generalization of kappas for multiple raters. Psychological Bulletin, 88, 322-328. doi:10.1037//0033-2909.88.2.322

Cooke, N. J., Gorman, J. C., Duran, J. L., \& Taylor, A. R. (2007). Team cognition in experiences command-and-control teams. Journal of Experimental Psychology: Applied, 13, 146-157. doi:10.1037/1076-898X.13.3.146

Cooke, N. J., Gorman, J. C., \& Winner, J. L. (2007). Team Cognition. In F. T. Durso (Ed.), Handbook of Applied Cognition (2 ed.). West Sussex: John Wiley \& Sons, Ltd.

Cooke, N. J., Kieckel, P. A., \& Helm, E. E. (2001). Measuring team knowledge during skill acquisition of a complex task. International Journal of Cognitive Ergonomics, 5, 297-315. doi:10.1207/S15327566IJCE0503_10

Cooke, N. J., Kiekel, P. A., Salas, E., Stout, R., Bowers, C., \& Cannon-Bowers, J. A. (2003). Measuring team knowledge: A window to the cognitive underpinnings of team performance. Group Dynamics: Theory, Research, and Practice, 7, 179-199. doi:10.1037/10892699.7.3.179

Cooke, N. J., Salas, E., Kiekel, P. A., \& Bell, B. (2004). Advances in measuring team cognition. In E. Salas \& S. M. Fiore (Eds.), Team cognition: Understanding the factors that drive process and performance. (pp. 83-106). Washington, DC, US: American Psychological Association. 
Craig, C., Klein, M. I., Griswold, J., Gaitonde, K., McGill, T., \& Halldorsson, A. (2013). Using cognitive task analysis to identify critical decisions in the laparoscopic environment. Human Factors, 54, 1025-1039. doi:10.1177/0018720812448393

Cramton, C. D. (2001). The mutual knowledge problem and its consequences for dispersed collaboration. Organization Science, 12. doi:1047-7039/01/1203/0346/\$05.D \{)

Crandall, B., Klein, G., \& Hoffman, R. (2006). Working minds: A practitioner's guide to cognitive task analysis. Cambridge: MIT Press.

Dalenberg, S., Vogelaar, A. L. W., \& Beersma, B. (2009). The Effect of a team strategy discussion on military team performance. Military Psychology, 21(1), S31-S46. doi:10.1080/08995600903249107

De Vries, R. E., \& Van den Hooff, B. (2006). Explaining knowledge sharing: The role of team communication styles, job satisfaction, and performance beliefs. Communication Research, 33(2), 115-135. doi:10.1177/0093650205285366

DeChurch, L. A., \& Mesmer-Magnus, J. R. (2010a). The cognitive underpinnings of effective teamwork: A meta-analysis. Journal of Applied Psychology, 95, 32-53. doi:10.1037/a0017328

DeChurch, L. A., \& Mesmer-Magnus, J. R. (2010b). Measuring shared team mental models: A metaanalysis. Group Dynamics: Theory, Research, and Practice, 14, 1-14. doi:10.1037/a0017455

Dionne, S. D., Sayama, H., Hao, C., \& Bush, B. J. (2010). The role of leadership in shared mental model convergence and team performance improvement: An agent-based computational model. The Leadership Quarterly, 21, 1035-1049. doi:10.1016/j.leaqua.2010.10.007

Driskell, J. E., Radtke, P. H., \& Salas, E. (2003). Virtual teams: Effects of technological mediation on team performance. Group Dynamics: Theory, Research, and Practice, 7, 297-323. doi:10.1037/1089-2699.7.4.297

Druskat, V. U., \& Pescosolido, A. T. (2002). The content of effective teamwork mental models in self-managing teams: Ownership, learning and heedful interrelating. Human Relations, 55, 283-314. doi:10.1177/0018726702553001

EATMP. (1999). Integrated task and job analysis of air traffic controllers. Phase 2. Task analysis of en-route controllers. (HUM.ET1.ST01.1000-REP-04). EUROCONTROL.

Edmondson, A. C. (1997). Group and organizational influences on team learning. Dissertation Abstracts International: Section B: The Sciences and Engineering, 57(10-B), 6647.

Edwards, B. D., Day, E. A., Arthur, W., \& Bell, S. T. (2006). Relationships among team ability composition, team mental models, and team performance. Journal of Applied Psychology, 91, 727-736. doi:10.1037/0021-9010.91.3.727

Ellis, A. P. J., \& Pearsall, M. J. (2011). Reducing the negative effects of stress in teams through crosstraining: A job demands-resources model. Group Dynamics: Theory, Research, and Practice, 15, 16-31. doi:10.1037/a0021070

Endsley, M. (1995). Towards a theory of situational awareness in dynamic systems. Human Factors, $37(1), 32-64$.

Entin, E. E., \& Serfaty, D. (1999). Adaptive team coordination. Human Factors, 41(2), 312-325.

Espevik, R., Johnsen, B. H., \& Eid, J. (2011). Communication and performance in co-located and distributed teams: An issue of shared mental models of team members? Military Psychology, 23, 616-638. doi:10.1080/08995605.2011.616792

Espevik, R., Johnsen, B. H., Eid, J., \& Thayer, J. F. (2006). Shared mental models and operational effectiveness: Effects on performance and team processes in submarine attack teams. Military Psychology, 18(3), 23-36. doi:10.1207/s15327876mp1803s_3

Espinosa, J. A., Lerch, F. J., \& Kraut, R. E. (2004). Explicit versus implicit coordination mechanisms and task dependencies: One size does not fit all. In E. Salas \& S. M. Fiore (Eds.), Team cognition: Understanding the factors that drive process and performance. (pp. 107-129). Washington, DC, US: American Psychological Association.

Fiore, S. M., Rosen, M. A., Smith-Jentsch, K. A., Salas, E., Letsky, M., \& Warner, N. (2010). Toward an understanding of macrocognition in teams: Predicting processes in complex collaborative contexts. Human Factors: The Journal of the Human Factors and Ergonomics Society, 52, 203-224. doi:10.1177/0018720810369807 
Fischer, U., McDonnell, L., \& Orasanu, J. (2007). Linguistic correlates of team performance: toward a tool for monitoring team functioning during space missions. Aviation, Space, and Environmental Medicine, 78(5), B86-B95.

Fleiss, J. L. (1971). Measuring nominal scale agreement among many raters. Psychological Bulletin, 76, 378-382. doi: 10.1037/h0031619

Gersick, C. J. G. (1988). Time and transition in work teams: Toward a new model of group development. Academy of Management Journal, 31, 9-41. doi:10.2307/256496

Good, P. I. (2000). Permutation test: a practical guide to resampling methods for testing hypotheses (2nd ed.). New York, NY: Springer-Verlag.

Gorman, J. C., Cooke, N. J., \& Amazeen, P. G. (2010). Training adaptive teams. Human Factors, 52, 295-307. doi:10.1177/0018720810371689

Greitemeyer, T., Schulz-Hardt, S., Brodbeck, F. C., \& Frey, D. (2006). Information sampling and group decision making: The effects of an advocacy decision procedure and task experience. Journal of Experimental Psychology: Applied, 12, 31-42.

Gross, N., \& Kluge, A. (2012). "Why should I share what I know?" - Antecedents for enhancing knowledge-sharing behavior and its impact on shared mental models in steel production. Paper presented at the Human Factors and Ergonmics Society 56th Annual Meeting, Boston, Massachusetts.

Gross, N., \& Kluge, A. (2014). Predictors of knowledge-sharing behavior for teams in extreme environments: An example from the steel industry. Journal of Cognitive Engineering and Decision Making, 8(4), 352-373. doi:10.1177/1555343414540656

Gurtner, A., Tschan, F., Semmer, N. K., \& Nägele, C. (2007). Getting groups to develop good strategies: Effects of reflexivity interventions on team process, team performance, and shared mental models. Organizational Behavior and Human Decision Processes, 102, 127-142. doi:10.1016/j.obhdp.2006.05.002

Hacker, W. (1978). Allgemeine Arbeits- und Ingenieurspsychologie: Psychische Struktur und Regulation von Arbeitstätigkeiten. Bern: Huber Verlag.

Hacker, W. (1986). Arbeitspsychologie: Psychische Regulation van Arbeitstätigkeiten. Bern: Huber Verlag.

Hacker, W. (2003). Action Regulation Theory: A practical tool for the design of modern work processes? European Journal of Work and Organizational Psychology, 12(2), 105-130. doi:10.1080/13594320344000075

Hackman, J. R. (2003). Learning more by crossing levels: Evidence from airplanes, hospitals, and orchestras. Journal of Organizational Behavior, 24, 905-922. doi:10.1002/job.226

Hess, S. M., MacMillan, J., Serfaty, D., \& Elliot, L. (1999). From cognitive task analysis to simulation: Developing a synthetic team task for AWACS Weapons Directors. Paper presented at the Command and Control Research and Technology Symposium, Newport, RI.

Hinsz, V. B. (2004). Metacognition and mental models in groups: An illustration with metamemory of group recognition memory. In E. Salas \& S. M. Fiore (Eds.), Team cognition: Understanding the factors that drive process and performance. (pp. 33-58). Washington, DC, US: American Psychological Association.

Hockey, G. R. J. (1997). Compensatory control in the regulation of human performance under stress and high workload: A cognitive-energetical framework. Biological Psychology, 45(1), 73-93. doi:10.1016/S0301-0511(96)05223-4

Hoffman, R. R., Shadbolt, N. R., Burton, A. M., \& Klein, G. (1995). Eliciting knowledge from experts: A methodological analysis. Organizational Behavior and Human Decision Processes, 62(2), 129-158.

ICAO. (1996). Rules of the Air and Air Traffic Services. Procedures for Air Navigation Services. (Doc 4444-RAC/501). International Civil Aviation Organization.

ICAO. (2001). Annex 1 to the Convention on International Civil Aviation: Personnel licensing. (Amdt 169-B).

Ilgen, D. R. (1999). Teams embedded in organizations. American Psychologist, 54, 129-139.

Jehn, K. A., Northcraft, G. B., \& Neale, M. A. (1999). Why differences make a difference: A field study of diversity, conflict and performance in workgroups. Administrative Science Quarterly, 44, 741-763. doi:10.2307/2667054 
Jo, I.-H. (2012). Shared Mental Models on the performance of e-learning content development teams. Educational Technology \& Society, 15(1), 289-297.

Johnson-Laird, P. (1983). Mental Models. Cambridge, MA: Harvard University Press.

Kahneman, D. (1973). Attention and effort. Englewood Cliffs, NJ: Prentice Hall.

Kanki, B. G., Folk, V. G., \& Irwin, C. M. (1991). Communication variations and aircrew performance. The International Journal of Aviation Psychology, 1, 149-162. doi:10.1207/s15327108ijap0102_5

Kellermanns, F. W., Floyd, S. W., Pearson, A. W., \& Spencer, B. (2005). The contingent effect of constructive confrontation on the relationship between shared mental models and decision quality. Journal of Organizational Behavior, 29, 119-137. doi:10.1002/job.497

Kellermanns, F. W., Floyd, S. W., Pearson, A. W., \& Spencer, B. (2008). The contingent effect of constructive confrontation on the relationship between shared mental models and decision quality. Journal of Organizational Behavior, 29, 119-137. doi:10.1002/job.497

Kennedy, D., \& McComb, S. (2010). Merging internal and external processes: Examining the mental model convergence process through team communication. Theoretical Issues in Ergonomics Science, 11, 340-358. doi:10.1080/14639221003729193

Keyton, J., \& Beck, S. J. (2010). Perspectives: Examining communication as macrocognition in STS. Human Factors: The Journal of the Human Factors and Ergonomics Society, 52(2), 335-339. doi: $10.1177 / 0018720810371338$

Kirschner, P. A., \& Van Merriënboer, J. (2008). Ten steps to complex learning: A new approach to instruction and instructional design. In T. L. Good (Ed.), 21st century education: A reference handbook (pp. 244-253). Thousand Oaks, CA: Sage.

Klein, G., \& Militello, L. (2001). Some guidelines for conducting a cognitive task analysis. In E. Salas (Ed.), Advances in human performance and cognitive engineering research (Vol. 1). Amsterdam: JAI.

Klimoski, R. J., \& Mohammed, S. (1994). Team mental model: Construct or metaphor? Journal of Management, 20, 403-437. doi:10.1177/014920639402000206

Kontogiannis, T., \& Malakis, S. (2013). Strategies in controlling, coordinating and adapting performance in air traffic control: modelling 'loss of control' events. Cognition Technology and Work, 15, 153-169. doi:10.1007/s10111-011-0209-0

Kozlowski, S. W. J., Gully, S. M., Nason, E. R., \& Smith, E. M. (1999). Developing adaptive teams: A theory of compilation and performance across levels and time. In D. R. Ilgen \& E. D. Pulakos (Eds.), The Changing Nature of Performance: Implications for Staffing, Motivation, and Development (pp. 240-292). San Francisco, CA: Jossey-Bass.

Kozlowski, S. W. J., \& Ilgen, D. R. (2000). A multilevel approach to theory and research in organizations: Contextual, temporal, and emergent processes. In K. J. Klein \& S. W. J. Kozlowski (Eds.), Multilevel theory, research, and methods in organizations: Foundations, extensions and new directions (pp. 3-90). San Francisco: Jossey-Bass.

Kozlowski, S. W. J., Watola, D. J., Jensen, J. M., Kim, B. H., \& Botero, I. C. (2009). Developing adaptive teams: A theory of dynamic team leadership. In E. Salas, G. F. Goodwin, \& C. S. Burke (Eds.), Team effectiveness in complex organizations: Cross-disciplinary perspectives and approaches (pp. 113-156). New York, NY: Routledge Academic.

Kraiger, K., Ford, J. K., \& Salas, E. (1993). Application of cognitive, skill-based, and affective theories of learning outcomes to new methods of training evaluation. Journal of Applied Psychology, 78, 311-328.

Kraiger, K., \& Wenzel, L. H. (1997). Conceptual development and empirical evaluation of measures of shared mental models as indicators of team effectiveness. In M. T. Brannick, E. E. Salas, \& C. Prince (Eds.), Team Performance Assessment and Measurement: Theory, Methods, and Applications. Mahwah, NJ: Lawrence Erlbaum Associates Publishers.

Kring, J. P. (2005). Communication modality and after action review performance in a distributed immersive virtual environment. Dissertation Abstracts International: Section B: The Sciences and Engineering, 66(1-B), 598.

LaHuis, D. M., \& Ferguson, M. W. (2009). The accuracy of significance tests for slope variance components in multilevel random coefficient models. Organizational Research Methods, 12, 418-435. doi:10.1177/1094428107308984 
Lancaster, J. A., \& Casali, J. G. (2008). Investigating pilot performance using mixed-modality simulated data link. Human Factors, 50, 183-193. doi:10.1518/001872008X250737

Landis, J. R., \& Koch, G. G. (1977). The measurement of observer agreement for categorical data. Biometrics, 33, $159-174$.

Lang, J. W. B., \& Bliese, P. D. (2009). General mental ability and two types of adaptation to unforeseen change: Applying discontinuous growth models to the task-change paradigm. Journal of Applied Psychology, 94, 411-428. doi:10.1037/a0013803

Langan-Fox, J., Anglim, J., \& Wilson, J. R. (2004). Mental models, team mental models, and performance: Process, development, and future directions. Human Factors and Ergonomics in Manufacturing, 14, 331-352. doi:10.1002/hfm.20004

Langan-Fox, J., Sankey, M. J., \& Canty, J. M. (2009). Human factors measurement for future air traffic control systems. Human Factors, 51, 595-637. doi:10.1177/0018720809355278

Langan-Fox, J., Wirth, A., Code, S., Langfield-Smith, K., \& Wirth, A. (2001). Analyzing shared and team mental models. International Journal of Industrial Ergonomics, 28(2), 99-112. doi:10.1016/S0169-8141(01)00016-6

Lee, M., \& Johnson, T. E. (2008). Understanding the effects of team cognition associated with complex engineering tasks: Dynamics of shared mental models, task-SMM, and team-SMM. Performance Improvement Quarterly, 21, 73-95. doi:10.1002/piq.20032

Lee, W.-C., \& Rodgers, J. L. (1998). Bootstrapping correlation coefficients using univariate and bivariate sampling. Psychological Methods, 3, 91-103.

LePine, J. A. (2005). Adaptation of teams in response to unforeseen change: Effects of goal difficulty and team composition in terms of cognitive ability and goal orientation. Journal of Applied Psychology, 90, 1153-1167. doi:10.1037/0021-9010.90.6.1153

Levesque, L. L., Wilson, J. M., \& Wholey, D. R. (2001). Cognitive divergence and shared mental models in software development project teams. Journal of Organizational Behavior, 22, 135144. doi: $10.1002 /$ job.87

Lim, B.-C., \& Klein, K. J. (2006). Team mental models and team performance: A field study of the effects of team mental model similarity and accuracy. Journal of Organizational Behavior, 27, 403-418. doi:10.1002/job.387

Lindlof, T. R., \& Taylor, B. C. (2011). Qualitative communication research (3 ed.). Thousand Oaks, CA: Sage Publications, Inc.

MacMillan, J., Entin, E. E., \& Serfaty, D. (2004). Communication overhead: The hidden cost of team cognition. In E. Salas \& S. M. Fiore (Eds.), Team cognition: Understanding the factors that drive process and performance. (pp. 61-82). Washington, DC, US: American Psychological Association.

Malakis, S., Kontogiannis, T., \& Kirwan, B. (2010a). Managing emergencies and abnormal situations in air traffic control (part I): Taskwork strategies. Applied Ergonomics, 41, 620-627. doi:10.1016/j.apergo.2009.12.019

Malakis, S., Kontogiannis, T., \& Kirwan, B. (2010b). Managing emergencies and abnormal situations in air traffic control (part II): Teamwork strategies. Applied Ergonomics, 41, 628-635. doi:10.1016/j.apergo.2009.12.018

Marks, M. A., Mathieu, J. E., \& Zaccaro, S. J. (2001). A temporally based framework and taxonomy of team processes. Academy of Management Review(3), 356-376. doi:10.5465/AMR.2001.4845785

Marks, M. A., Sabella, M. J., Burke, C. S., \& Zaccaro, S. J. (2002). The impact of cross-training on team effectiveness. Journal of Applied Psychology, 87, 3-13. doi:10.1037//0021-9010.87.1.3

Marks, M. A., Zaccaro, S. J., \& Mathieu, J. E. (2000). Performance implications of leader briefings and team-interaction training for team adaptation to novel environments. Journal of Applied Psychology, 85, 971-986. doi:10.1037/0021-9010.85.6.971

Marsh, J. K., \& Ahn, W.-k. (2012). Memory for patient information as a function of experience in mental health. Applied Cognitive Psychology, 26(3), 462-474. doi:10.1002/acp.2832

Mathieu, J. E., Heffner, T. S., Goodwin, G. F., Cannon Bowers, J. A., \& Salas, E. (2005). Scaling the quality of teammates' mental models: Equifinality and normative comparisons. Journal of Organizational Behavior, 26, 37-56. doi:10.1002/job.296 
Mathieu, J. E., Heffner, T. S., Goodwin, G. F., Salas, E., \& Cannon Bowers, J. A. (2000). The influence of shared mental models on team process and performance. Journal of Applied Psychology, 85, 273-283. doi:10.1037//0021-9010.85.2.273

Mathieu, J. E., Rapp, T. L., Maynard, M. T., \& Mangos, P. M. (2010). Interactive effects of team and task shared mental models as related to air traffic controllers' collective efficacy and effectiveness. Human Performance, 23, 22-40. doi:10.1080/08959280903400150

McComb, S. (2007). Mental model convergence: The shift from being an individual to being a team member. In F. Dansereau \& F. Yammarino (Eds.), Multi-level issues in organizations and time (pp. 95-147). Oxford, UK: Elsevier Science.

McComb, S., Kennedy, D., Perryman, R., Warner, N., \& Letsky, M. (2010). Temporal patterns of mental model convergence: Implications for distributed teams interacting in electronic collaboration spaces. Human Factors, 52, 264-281. doi:10.1177/0018720810370458

McIntyre, H. H., \& Foti, R. J. (2013). The impact of shared leadership on teamwork mental models and performance in self-directed teams. Group Processes \& Intergroup Relations, 16, 46-57. doi: $10.1177 / 1368430211422923$

McIntyre, R. M., \& Salas, E. (1995). Measuring and managing for team performance: Emerging principles from complex environments. In R. A. Guzzo \& E. Salas (Eds.), Team effectiveness and decision making in organizations (pp. 9-45). San Francisco, CA: Jossey Bass.

Mesmer-Magnus, J. R., \& DeChurch, L. A. (2009). Information sharing and team performance: A meta-analysis. Journal of Applied Psychology, 94(2), 535-546. doi:10.1037/a0013773

Metzger, U., \& Parasuraman, R. (2006). Effects of automated conflict cuing and traffic density om air traffic controller performance and visual attention in a datalink environment. The International Journal of Aviation Psychology, 16, 343-362.

Militello, L. G., \& Hutton, R. J. B. (1998). Applied cognitive task analysis (ACTA): A practitioner's toolkit for understanding cognitive task demands. Ergonomics, 41(11), 1618-1641. doi: $10.1080 / 001401398186108$

Mohammed, S., \& Dumville, B. C. (2001). Team mental models in a team knowledge framework: Expanding theory and measurements across disciplinary boundaries. Journal of Organizational Behavior, 22, 89-106. doi:10.1002/job.86

Mohammed, S., Ferzandi, L., \& Hamilton, K. (2010). Metaphor no more: A 15-year review of the team mental model construct. Journal of Management, 36, 876-910. doi:DOI: $10.1177 / 0149206309656804$

Mohammed, S., Klimoski, R. J., \& Rentsch, J. R. (2000). The measurement of team mental models: We have no shared schema. Organizational Research Methods, 3, 123-165. doi:10.1177/109442810032001

Murphy, K. R. (1989). Is the relationship between cognitive ability and job performance stable over time? Human Performance, 2(3), 183-200. doi:10.1207/s15327043hup0203_3

Neal, A., Flach, J., Mooij, M., Lehmann, S., Stankovic, S., \& Hasenbosch, S. (2011). Envisaging the future air traffic management system. The International Journal of Aviation Psychology, 21(1), 16-34. doi:10.1080/10508414.2011.537557

Niessen, C., \& Eyferth, K. (2001). A model of the air traffic contoller's picture. Safety Science, 37, 187-202.

Niessen, C., Leuchter, S., \& Eyferth, K. (1998). A psychological model of air traffic control and its implementation. Paper presented at the Second European Conference of Cognitive Modelling, Nottingham, UK.

Okhuysen, G. A., \& Eisenhardt, K. M. (2002). Integrating knowledge in groups: How formal interventions enable felxibility. Organization Science, 13(4), 370-386. doi:10.1287/orsc.13.4.370.2947

Okhuysen, G. A., \& Waller, M. J. (2002). Focusing on midpoint transitions: An analysis of boundary conditions. Journal of Management Journal, 45(5), 1056-1065. doi: 10.2307/3069330

Oprins, E., Burggraaff, E., \& Van Weerdenburg, H. (2006). Design of a competence-based assessment system for air traffic control training. The International Journal of Aviation Psychology, 16, 297-320. 
Peterson, E., Mitchell, T. R., Thompson, L., \& Burr, R. (2000). Collective efficacy and aspects of shared mental models as predictors of performance over time in work groups. Group Processes and Intergroup Relations(3), 296-316. doi:10.1177/1368430200033005

Pinheiro, J. C., Bates, D. M., DebRoy, S., \& Sarkar, D. (2011). nlme: Linear and nonlinear mixed effects models (Version 3.1-101) [Computer software]. Vienna: R Foundation for Statistical Computing. Retrieved June 2011, from http://cran.r-project.org/.

Porter, C. O., Hollenbeck, J. R., Ilgen, D. R., Ellis, A. P., West, B. J., \& Moon, H. (2003). Backing up behaviors in teams: The role of personality and legitimacy of need. Journal of Applied Psychology, 88, 391-403. doi:10.1037/0021-9010.88.3.391

Randall, K. R., Resick, C. J., \& DeChurch, L. A. (2011). Building team adaptive capacity: The roles of sensegiving and team composition. Journal of Applied Psychology, 96, 525-540. doi:10.1037/a0022622

Rentsch, J. R., \& Hall, R. J. (1994). Members of great teams think alike: A model of the effectiveness and schema similarity among team members. Advances in Interdisciplinary Studies of Work Teams, 223-261.

Rentsch, J. R., \& Klimoski, R. J. (2001). Why do 'great minds' think alike?: Antecedents of team member schema agreement. Journal of Organizational Behavior, 22, 107-120. doi:10.1002/job.81

Rentsch, J. R., Small, E. E., \& Hanges, P. J. (2008). Cognitions in organizations and teams: What is the meaning of cognitive similarity? In B. Smith (Ed.), The people make the place (pp. 129157). Mahwah, NJ: Lawrence Erlbaum.

Resick, C. J., Dickson, M. W., Mitchelson, J. K., Allison, L. K., \& Clark, M. A. (2010). Team composition, cognition, and effectiveness: Examining mental model similarity and accuracy. Group Dynamics: Theory, Research, and Practice, 25, 274-191. doi:10.1037/a0018444

Rico, R., Sánchez-Manzanares, M., Gil, F., \& Gibson, C. (2008). Team implicit coordination processes: A team knowledge-based approach. The Academy of Management Review, 33, 163-184. doi:10.5465/AMR.2008.27751276

Rosen, M. A., Bedwell, W. L., Wildman, J. L., Fritzsche, B. A., Salas, E., \& Burke, C. S. (2011). Managing adaptive performance in teams: Guiding principles and behavioral markers for measurement. Human Resource Management Review, 21(2), 107-122. doi:10.1016/j.hrmr.2010.09.003

Ross, S., \& Allen, N. (2012). Examining the convergent validity of shared mental model measures. Behavior Research Methods, 44, 1052-1062. doi:10.3758/s13428-012-0201-5

Rouse, W. B., \& Morris, N. M. (1986). On looking into the black box: Prospects and limits in the search for mental models. Psychological Bulletin, 100, 349-363. doi:10.1037//00332909.100.3.349

Salas, E., Cannon Bowers, J. A., \& Johnston, J. H. (1997). How can you turn a team of experts into an expert team?: Emerging training strategies. In C. E. Zsambok \& G. Klein (Eds.), Expertise: Research and applications. (pp. 359 370). Hillsdale, NJ, England: Lawrence Erlbaum Associates, Inc.

Salas, E., \& Fiore, S. M. (2004). Team cognition: Understanding the factors that drive process and performance. In E. Salas \& S. M. Fiore (Eds.), Why team cognition? An overview. (pp. 3-8). Washington, Dc: American Psychological Association.

Salas, E., Nichols, D. R., \& Driskell, J. E. (2007). Testing three team training strategies in intact teams: A meta-analysis. Small Group Research, 38, 471-488. doi:10.1177/1046496407304332

Salas, E., Rosen, M. A., Burke, C. S., Nicholson, D., \& Howse, W. R. (2007). Markers for enhancing team cognition in complex environments: The power of team performance diagnosis. Aviation, Space, and Environmental Medicine, 78(5), B77 - B85.

Salas, E., Rosen, M. A., Held, J. D., \& Weissmuller, J. J. (2009). Performance measurement in simulation-based training: A review and best practices. Simulation \& Gaming, 40, 328-376. doi:10.1177/1046878108326734

Salas, E., Sims, D. E., \& Burke, C. S. (2005). Is there a "big five" in teamwork? Small Group Research, 36(5), 555-599. 
Salas, E., Wilson, K. A., Burke, C. S., \& Wightman, D. C. (2006). Does crew resource management training work? An update, an extension, and some critical needs. Human Factors, 48, 392412. doi:10.1518/001872006777724444

Salas, E., Wilson, K. A., \& Edens, E. (2009). Crew resource management. London: Ashgate.

Sander, P. C., van Doorn, R. R. A., van der Pal, J., \& Zijlstra, F. R. H. (2015). Team adaptation to an unforeseen system failure: Limits of the potential aids of shared knowledge and standardized communication. European Journal of Work and Organizational Psychology. doi:10.1080/1359432X.2015.1006199

Schraagen, J. M., Chipman, S., \& Shalin, V. (2000). Cognitive task analysis. Mahwah, NJ: Lawrence Erlbaum.

Seamster, T., Redding, R. E., Cannon, R. C., Ryder, J. M., \& Purcell, J. A. (1993). Cognitive task analysis of expertise in air traffic control. The International Journal of Aviation Psychology, 3(4), 257-283.

Seamster, T., Redding, R. E., \& Kaempf, G. (1997). Applied cognitive task analysis in aviation. Aldershot, UK: Ashgate Publishing Ltd.

SESAR. (2012). The roadmap for sustainable air traffic management: European ATM master plan. Retrieved from https://www.atmmasterplan.eu/.

Sharples, S., Stedmon, A. W., Cox, G., Nicholls, A., Shuttleworth, T., \& Wilson, J. (2007). Flightdeck and Air Traffic Control Collaboration Evaluation (FACE): Evaluating aviation communication in the laboratory and field. Applied Ergonomics, 38, 399-407. doi:10.1016/j.apergo.2007.01.012

Sikorski, E. G., Johnson, T. E., \& Ruscher, P. H. (2012). Team knowledge sharing intervention effects on team shared mental models and student performance in an undergraduate science course. Journal of Science Education and Technology, 21(6), 641-651. doi:10.1007/s10956-0119353-9

Simon, H. A., \& Chase, W. G. (1973). Skill in chess. American Scientist, 61, 394-403.

Smith-Jentsch, K. A. (2009). Measuring team-related cognition: The devil is in the details. In E. Salas, G. F. Goodwin, \& C. S. Burke (Eds.), Team effectiveness in complex organizations. New York, NY: Taylor \& Francis.

Smith-Jentsch, K. A., Campbell, G., Milanovich, D., \& Reynolds, A. (2001). Measuring teamwork mental models to support training needs assessment, development, and evaluation: Two empirical studies. Journal of Organizational Behavior, 22, 179-194. doi:10.1002/job.88

Smith-Jentsch, K. A., Cannon-Bowers, J. A., Tannenbaum, S. I., \& Salas, E. (2008). Guided team self-correction: Impacts on team mental models, processes, and effectiveness. Small Group Research, 39, 303-327. doi:10.1177/1046496408317794

Smith-Jentsch, K. A., Kraiger, K., Cannon-Bowers, J. A., \& Salas, E. (2009). Do familiar teammates request and accept more backup? Transactive memory in air traffic control. Human Factors, 51, 181-192. doi:10.1177/0018720809335367

Smith-Jentsch, K. A., Mathieu, J. E., \& Kraiger, K. (2005). Investigating linear and interactive effects of shared mental models on safety and efficiency in a field setting. Journal of Applied Psychology, 90, 523-535. doi:10.1037/0021-9010.90.3.523

Snijders, T. A. B., \& Bosker, R. J. (1999). Multilevel analysis: An introduction to basic and advanced multilevel modeling. London: Sage Publications.

Spencer, D. (2009). Card sorting - Designing usable categories. Brooklyn, NY: Rosenfeld Media, LLC.

Stachowski, A. A., Kaplan, S. A., \& Waller, M. J. (2009). The benefits of flexible team interaction during crises. Journal of Applied Psychology, 94, 1536-1543. doi:10.1037/a0016903

Stasser, G., \& Titus, W. (1985). Pooling of unshared information in group decision making: Biased information sampling during discussion. Journal of Personality and Social Psychology, 48, $1467-1478$.

Stasser, G., \& Titus, W. (1987). Effects of information load and percentage of shared information on the dissemination of unshared information during group discussion. Journal of Personality and Social Psychology, 53, 81-93. 
Stedmon, A. W., Sharples, S., Littlewood, R., Cox, G., Patel, H., \& Wilson, J. R. (2007). Datalink in air traffic management: Human factors issues in communications. Applied Ergonomics, 38, 473-480. doi:10.1016/j.apergo.2007.01.013

Sternberg, R. J. (2008). Cognitive psychology (5th ed.). Belmont, CA: Cengage.

Stout, R. J., Cannon Bowers, J. A., Salas, E., \& Milanovich, D. M. (1999). Planning, shared mental models, and coordinated performance: An empirical link is established. Human Factors, 41(1), 61-71.

Summers, J. K., Humphrey, S. E., \& Ferris, G. R. (2012). Team member change, flux in coordination, and performance: Effects of strategic core roles, information transfer, and cognitive ability. Academy of Management Journal, 55, 314-338. doi:10.5465/amj.2010.0175

Takane, Y., Young, F. W., \& de Leeuw, J. (1976). Nonmetric individual differences scaling: An alternating least squares method with optimal scaling features. Psychometrika, 42, 7-67.

Tannenbaum, S. I., \& Cerasoli, C. P. (2013). Do team and individual debriefs enhance performance? A meta-analysis. Human Factors, 55(1), 231-245. doi:10.1177/0018720812448394

Thompson, L. F., \& Coovert, M. D. (2003). Teamwork online: The effects of computer conferencing on perceived confusion, satisfaction, and postdiscussion accuracy. Group Dynamics: Theory, Research, and Practice, 7, 135-151. doi:10.1037/1089-2699.7.2.135

Uitdewilligen, S., Waller, M. J., \& Pitariu, A. H. (2013). Mental model updating and team adaptation. Small Group Research, 44, 127-158. doi:10.1177/1046496413478205

Uitdewilligen, S., Waller, M. J., \& Zijlstra, F. R. H. (2010). Team cognition and adaptability in dynamic settings: A review of pertinent work. In G. P. Hodgkinson \& J. K. Ford (Eds.), International Review of Industrial and Organizational Psychology (Vol. 25). Oxford, UK: Wiley-Blackwell.

Ure, J., Dewar, R., Pooley, R., Lloyd, A., \& Jaegersberg, J. (2004). Mental models as enablers of knowledge sharing and decision-making in the design of collaborative networked environments. In L. Camarinha-Matos (Ed.), Virtual Enterprises and Collaborative Networks (Vol. 149, pp. 597-606): Springer US.

Van Avermaete, J. A. G., \& Kruijsen, E. A. C. (1998). The evaluation of non-technical skills of multipilot aircrew in relation to the JAR-FCL requirements. (NLR-CR-98443). Amsterdam: National Aerospace Laboratory.

Van den Bossche, P., Gijselaers, W., Segers, M., Woltjer, G., \& Kirschner, P. (2011). Team learning: building shared mental models. Instructional Science, 39, 283-301. doi:10.1007/s11251-0109128-3

Van der Pal, J. (2005). The MEC approach: Observations and review. NLR-CR-2005-306. Retrieved from

Van der Pal, J., \& Justen, P. (2011). TeamTris: A Research and Training Paradigm for Team Work in Dynamic Environments. Paper presented at the 18th International Conference on Technology Supported Learning \& Training, Berlin.

Van Merriënboer, J., \& Dijkstra, S. (1997). The four component instructional design model for training complex cognitive skills. In R. D. Tennyson \& F. Schott (Eds.), Instructional design: International perspectives. Theory and research. (Vol. 1, pp. 427-445). Hilsdale, NJ: The four component instructional design model for training complex cognitive skills.

Voller, L., \& Low, I. (2004). Impact of automation on future controller skill requirements and a framework for their prediction. Retrieved from Brussels, Belgium:

Volpe, C. E., Cannon-Bowers, J. A., Salas, E., \& Spector, P. E. (1996). The impact of cross-training on team functioning: An empirical investigation. Human Factors, 38, 87-100. doi:10.1518/001872096778940741

Waller, M. J. (1999). The timing of adaptive group responses to nonroutine events. Academy of Management Journal, 42(2), 127-137.

Waller, M. J., Gupta, N., \& Giambatista, R. C. (2004). Effects of adaptive behaviors and shared mental models on control crew performance. Management Science, 50, 1534-1544. doi: $10.1287 / \mathrm{mnsc} .1040 .0210$

Wang, S., \& Noe, R. A. (2010). Knowledge sharing: A review and directions for future research. Human Resource Management Review, 20, 115-131. doi:10.1016/j.hrmr.2009.10.001 
Webber, S. S., Chen, G., Payne, S. C., Marsh, S. M., \& Zaccaro, S. J. (2000). Enhancing team mental model measurement with performance appraisal practices. Organizational Research Methods, 4, 307-322. doi:10.1177/109442810034001

Wei, J., \& Salvendy, G. (2004). The cognitive task analysis methods for job and task design: Review and reappraisal. Behaviour \& Information Technology, 23(4), 273-299. doi:10.1080/01449290410001673036

Weick, K. E. (1985). Cosmos vs. chaos: Sense and nonsense in electronic contexts. Organizational Dynamics, 14(2), 51-64.

Weick, K. E. (1990). The vulnerable system: An analysis of the Tenerife air disaster. Journal of Management, 16, 571-593. doi:10.1177/014920639001600304

Weick, K. E., \& Roberts, K. H. (1993). Collective mind in organizations: Heedful interrelating on flight decks. Administrative Science Quarterly, 38(3), 357-381.

Wilson, K. A., Salas, E., Priest, H. A., \& Andrews, D. (2007). Errors in the heat of battle: Taking a closer look at shared cognition breakdowns through teamwork. Human Factors: The Journal of the Human Factors and Ergonomics Society, 49, 243-256. doi:10.1518/001872007X312478

Xiang, C., Lub, Y., \& Gupta, S. (2013). Knowledge sharing in information system development teams: examining the impact of shared mental model from a social capital theory perspective. Behaviour \& Information Technology, 32(10), 1024-1040. doi:10.1080/0144929X.2012.745901

Xin, W., \& Zhiming, W. (2005). Antecedents and Consequences of Shared Mental Model in Work Teams. Acta Psychologica Sinica, 37(4), 542-549.

Yang, H.-D., Kang, H.-R., \& Mason, R. M. (2008). An exploratory study in meta skills in software development teams: Antecedent cooperation skills and personality for shared mental models. European Journal of Information Systems, 17, 47-61. doi:10.1057/palgrave.ejis.3000730

Zachary, W. W., Ryder, J. M., \& Hicinbothom, J. H. (1998). Cognitive task analysis and modeling of decision making in complex environments. In J. A. Cannon-Bowers \& E. Salas (Eds.), Making decisions under stress: Implications for individual and team training. (pp. 315-344). Washington, DC US: American Psychological Association.

Zellmer-Bruhn, M., Waller, M. J., \& Ancona, D. (2004). The effect of temporal entrainment on the ability of teams to change their routines. In S. Blount (Ed.), Research on managing groups and teams (pp. 135-158). Oxford, UK: Elsevier.

Zijlstra, F. R. H., Waller, M. J., \& Phillips, S. I. (2012). Setting the tone: Early interaction patterns in swift-starting teams as a predictor of effectiveness. European Journal of Work and Organizational Psychology, 21, 749-777. doi:10.1080/1359432X.2012.690399 



\section{Valorization}

\section{Economic relevance}

The present dissertation has economic relevance as it contributes to improve teamwork and communication in teams by helping team members to develop a shared mental model. This is an important contribution to modern economy as teamwork and a shared understanding of the task among team members is increasingly crucial to accomplish complex tasks in many work settings. For example, in aviation there are often few opportunities for team members to share information that not directly pertains to the tasks but that may still help these team members to understand each other's behaviour. This specifically applies when team members work at different locations and communicate via text-based messages, such as datalink. These teams need (organizational) support to exchange relevant information, develop a common picture of the situation and work smoothly toward a common goal. The findings from this dissertation suggest ways to support teams in this process and provides practical recommendations for active and guided knowledge sharing. Chapters 4 and 5 give detailed descriptions on the design of such active and guided knowledge sharing in different circumstances. These chapters demonstrate how knowledge sharing can result in more accurate and more similar knowledge structures thereby contributing to efficient teamwork.

This dissertation is also relevant to economy as organizations and practitioners can use it as a guideline on how to identify team members' shared mental models in dynamic environments. This is important as shared mental models are cognitive constructs that first need to be elicited, analysed and interpreted. Chapters 2 and 3 outline how a cognitive task analysis and card sorting tasks allow to get insights into the mental knowledge structures of individuals and to operationalize the similarity of these knowledge structures between team members. Specifically, these chapters describe how to conduct a cognitive task analysis and a card sorting task in air traffic controllers and list the general advantages and disadvantages of both methods.

In general, improving shared mental models and teamwork among team members can have more far-reaching economic and social relevance. For instance, effective teamwork between air traffic controllers and cockpit crews can positively affect safety in aviation, while inefficient teamwork potentially leads to incidents and accidents in air traffic. Thus, through helping team members to work together more efficiently through operational measures or enhanced training we may be able to further increase safety. In addition, improving teamwork through facilitating shared mental model development may positively affect efficiency. For example, smooth cooperation among air traffic control, airlines and airport operations can lead to more environmentally friendly traffic flow and more service-oriented management of passengers. The results of the present studies can guide the design and 
implementation of training for these purposes in aviation and other complex work settings such as health care.

Improving safety and efficiency in aviation becomes particularly interesting in the future in which the European Union foresees the harmonization of the European airspace ${ }^{9}$. To achieve this harmonization, all European parties will need to engage in collaborative decision making and advanced automation will help them in this process. Team members with an effective shared mental model will more easily come to collaborative and safe decisions. In addition, if automation fails, they will still be able to work together effectively despite the changed situation.

\section{Target group}

A number of the insights in this dissertation are particularly valuable for the target group of air traffic controllers. The previous paragraphs describe a number of examples of how the different studies contribute to understand and improve shared knowledge and teamwork in the profession of air traffic control. Chapters 2 and 3 specifically address the content and similarity of mental models of air traffic controllers.

The subsequent chapters apply to complex teams in general. Complex teams also work in the military (e.g. combat teams), in health care (e.g. surgery teams), in the transportation and (nuclear) power industry (e.g. control room teams). They have a number of characteristics in common that make a shared mental model particular relevant for efficient teamwork. Complex teams must process and share large amounts of new information in a restricted time period to make decisions collaboratively and adapt quickly to changing and maybe unforeseen situations. In such situations, complex teams have limited possibilities to communicate explicitly while mistakes often have major consequences. Complex teams overcome these situations and perform the required tasks because they benefit from a shared mental model, which allows them to anticipate each other's behaviour and maintain a similar picture of the evolving situation.

\section{Activities and products}

A tangible product that evolves from the present research activities is the tool TeamTris. TeamTris is a dynamic environment in which multiple team members interact in a cooperative game world. It is easy to learn but hard to master, which intrinsically motives the user. TeamTris was used in the experiments in this dissertation to study teamwork, shared mental model development, team adaptation and communication. In addition, the tool can easily be adapted to investigate other study variables (e.g. mental effort) in multiple experimental conditions, as well as to support training and selection of professionals.

\footnotetext{
${ }^{9}$ More information on the harmonization of the European airspace is available at the project site of the Single European Sky ATM Research: http://www.sesarju.eu/
} 
As a selection tool, TeamTris could be applied to test qualifications for specific professions such as air traffic control. While this assumptions needs to be validated, there is some indication for the usefulness of TeamTris during selection procedures. During a small pilot study parallel this research, air traffic control trainees played TeamTris. It appeared that they were able to learn and play the game quicker than the average team of university students. The air traffic control trainees reached a high performance score within a short amount of time and avoided penalty points successfully. This small study implied that TeamTris may distinguish individuals that have been selected on specific competences (e.g. planning, decision making, teaming) and individuals that have not been selected on these competences.

TeamTris could also be applied during training of professionals. First, TeamTris can be applied to address team aspects much earlier during training of operational tasks than it is currently common. Due to the complexity of these operational tasks (e.g. air traffic control) trainees often first have to train individual skills before they learn to work effectively in a team. Since TeamTris is a simulated and abstract game environment, young trainees can discover and train the importance of a shared understanding and effective coordination before they are an expert on the actual task. This may help them later when they train on the workflow together with their senior colleagues.

A second useful training application of TeamTris is to further improve teamwork abilities of experts and to help them to adapt to (non-nominal) situations. Through TeamTris team members of an inefficient team can for example train communication skills, time management (i.e. when to provide information to each other) and smooth coordination in a safe environment. They can experience the impact of inefficient teamwork without fatal consequences and can train to change their behaviour and improve their skills. In addition, TeamTris allows individuals to train in unexpected situations such as a system failure. Although such situations are unusual it is extremely important that complex teams manage these situations adequately. Through TeamTris they can develop strategies of how to build a shared mental picture of the evolving situation, make plans how to adapt to the situation and jointly execute this plan. 



\section{Acknowledgement}

Fred, thank you for your incredible patience and confidence in my work. You always found positive words and motivated me throughout the whole research project. Due to your broad knowledge and expertise in Work and Organizational Psychology, I was able to study team processes in both teams of air traffic controllers and complex teams generally.

Robert en Jelke, thank you for being such great copromotors. Robert, you taught me how to write my ideas up explicitly, comprehensibly and in an interesting way. You helped me to improve this dissertation during countless sessions and while most of the time we worked at different locations, your 'door' was always open. You put a lot of time, work and effort into helping me finishing my Ph.D. and I am very thankful for this.

Jelke, you helped me to concentrate on the essentials. You are both a great scientist and a great practitioner. Your applied perspective supported me in searching for and clarifying the practical importance of my research. After one of my first 'tegenvallers' you told me that several more will follow and that it is important to accept and manage disappointments. I have thought of this conversation many times in the last couple of years and this often helped me to get back on track.

I would like to thank my 'paranimfen' Joris and Alicia. Joris, you are a great friend, an awesome partner in crime and you have always been a critical reader of my dissertation. Discussing my research during a cup of tea, or more frequently during a beer, was always pleasant and fruitful. Often you just kept me company when I worked long hours at the office. I felt less lonely and more productive with you on the other site of the desk. Thank you for your incredible support and your confidence in my competences. Thank you for the many nights we spent at Gent, in the park, in de Nachtwachtlaan or in a coffee house somewhere in our adopted home town.

Alicia, having you as a colleague and friend made the weeks of my experiments in Maastricht much more 'gezellig'. Thank you so much for always welcoming me at your house, where I was spoilt with great food and hospitality. I even had my own bedroom (I think we called it the Pia-room), which I admittedly but willingly shared with the cat sometimes. You always kept me up to date about what is going on at the university so that I felt part of the WOPers while working at NLR.

Thanks to my colleagues at NLR. Being an external PhD student at the ATTS department was a professional and personal gain. I learned a lot, for example about aviation, operator performance, even simulation, and about how to organize a proper 'lotjes trekken event'. Particularly, I like to thank Harrie for being a great manager. You always gave me the opportunity to realize my (research) ideas and you never doubted that I will succeed. Antoine, do you remember the first day we've met? I was immediately convinced that you would be the perfect roommate: a list of unknown Dutch words, a 
longitudinal study at our pin board, and many culinary and entertaining dinners together. Thank you. Arash, thank you for the excellent design of the cover of this dissertation. You gave me a hard time in making a choice between a number of nice covers. Your creativity and 'special mind', I'd like to call it, are unique and I hope you will always stay the way you are.

A number of other colleague's und professionals contributed to my research and this dissertation. I thank the members of the KDC research program 'HF in future ATM' for their constructive feedback during our meetings. Esther O., thank you for being my mentor early in my career and inspiring my enthusiasm in aviation. A special thanks to the many ATCOs at LVNL and Eurocontrol who participated in my studies, allowed observations in the control room and passionately explained everything about ATC I wanted to know. A special thanks to Otto S. and Emil V. from LVNL and Andrew S. from Eurocontrol

I would like to thank my friends and family for their incredible support. I had great roommates who all became friends. Thank you Merel, Kirstel, Julia, Joa, Petra, Fay and Anneloes (who I count as a kind of roommate) for making home a place of relaxation, fun and music. You gave me energy to keep going the next day. Thank you to my friends in Amsterdam with whom I played tennis, had BBQ in the park and rocked the city. Emy, thank you for being the best friend one can imagine since more than 30 years. When I started my PhD you said "Whatever Pia starts, she will finish". Thank you for your unquestionable trust, the help hotline whenever needed and for being such a wonderful friend.

Mama und Papa, you, in the first place made it possible that I studied and worked in the Netherlands, and that I have become a person who is able to find her way there. Anja, you always reminded me where home is. A special thanks to Papa and Doris for taking care of Carl so affectionately and wonderfully when I was writing my dissertation at the library. Finally yet importantly, I thank you, Frank. You helped me finishing my dissertation more than anyone else did. You kept pushing and motivating me again and again. You accepted no excuses and simultaneously you accepted that this meant that we had to back down as a couple and family. Thank you for your patience, your tolerance and your strong believe in me. 


\section{Curriculum vitae}

Pia Sander (nèe Justen) was born on June 191982 in Essen, Germany. She attended Gymnasium Waldstraße in Hattingen, Germany in 2002. After one year of studying at TU Dortmund and a summer Dutch course, she started with higher education at Maastricht University, the Netherlands. Pia received her Bachelor degree in Cognitive Psychology in 2006 and completed her Master degree (MSc.) in Applied Cognitive Psychology, Work and Organizational Psychology in 2007. In 2008, she started her Ph.D. research on shared mental models in complex teams as an external $\mathrm{PhD}$ student at the National Aerospace Laboratory, NLR in Amsterdam. She closely worked together with the Dutch Air Traffic Control as well as with Maastricht University to collect applied and experimental data. In 2012, Pia started working as a Human Factors and Training Expert at the Department of Training, Simulation and Operator Performance at NLR. After living in the Netherlands for 11 years, she moved back to Germany in 2014 and is currently on maternity leave. 
